Aus dem Institut für Diagnostische und Interventionelle

Neuroradiologie

(Prof. Dr. med. C. Riedel)

der Medizinischen Fakultät der Universität Göttingen

\title{
Sensitivität und Spezifität der Detektion von Vasospasmen nach SAB mittels früharterieller CTP-basierten 4D-CTA- Rekonstruktionen sowie konventioneller CTA
}

\author{
INAUGURAL-DISSERTATION \\ zur Erlangung des Doktorgrades \\ der Medizinischen Fakultät der \\ Georg-August-Universität zu Göttingen
}

vorgelegt von

Cathrin Möller

aus

Hannover

Göttingen 2021 
Dekan:

\section{Betreuungsausschuss}

Betreuer:

Ko-Betreuerin:

\section{Prüfungskommission}

Referent:

Ko-Referentin:

Drittreferent:

Datum der mündlichen Prüfung:
Prof. Dr. med. W. Brück

PD Dr. med. D. Behme

Prof. Dr. med. D. Mielke

PD Dr. Daniel Behme

Prof. Dr. Dorothee Mielke

Prof. Dr. Margarete Schön

26.01 .2022 
Hiermit erkläre ich, die Dissertation mit dem Titel "Sensitivität und Spezifität der Detektion von Vasospasmen nach SAB mittels früharterieller CTP-basierte 4D-CTA-Rekonstruktionen sowie konventioneller CTA" eigenständig angefertigt und keine anderen als die von mir angegebenen Quellen und Hilfsmittel verwendet zu haben.

Göttingen, den 09.01.2022

Cathrin Möller 


\section{Inhaltsverzeichnis}

Abbildungsverzeichnis................................................IV

Tabellenverzeichnis......................................................... V

Abkürzungsverzeichnis..................................................VI

1 Einleitung......................................................................1 1

1.1 Ziel dieser Arbeit........................................................... 6

2 Methodik.................................................................8 8

2.1 Patientenauswahl.................................................................. 8

2.2 Technik der bildgebenden Verfahren....................................... 10

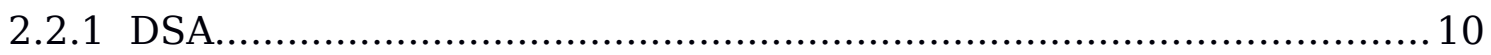

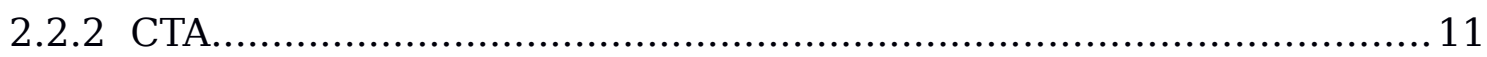

2.2.3 CTP und 4D-CTA aus CTP...................................................... 12

2.2.4 Geräte und Untersuchungsparameter................................. 13

2.3 Auswertung der Bildgebung.............................................. 14

2.4 Rating des Gefäßstatus........................................................... 18

2.4.1 Bewertete Gefäße............................................................. 18

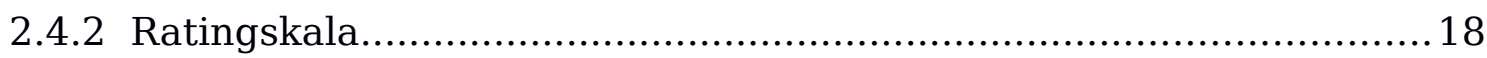

2.4.3 Unabhängige Auswertung.............................................. 20

2.5 Statistische Auswertung................................................. 20

2.5.1 Diagnose eines Vasospasmus............................................. 21

2.5.2 Bewertung des Vasospasmusgrades.................................... 21

2.5.3 Interrater-Reliabilität der Vasospasmusdetektion...........................22

2.5.4 Interrater-Reliabilität des Vasospasmusgrades...............................22

2.5.5 Aufschlüsselung nach Arterien.................................................. 22

2.5.6 Bewertung von Signifikanz.................................................... 23

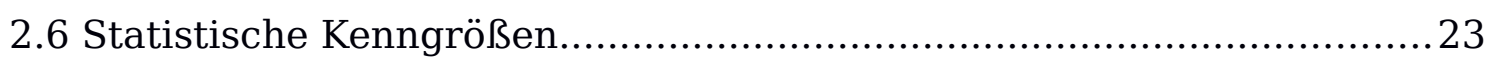

2.6.1 Sensitivität und Spezifität...................................................... 23

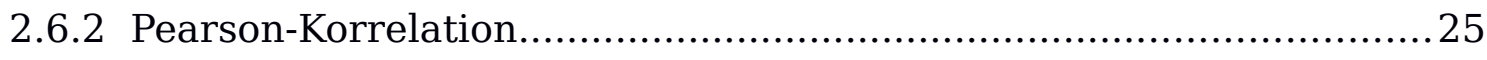

2.6.3 Interrater-Reliabilität...................................................... 25

3 Ergebnisse...................................................................27

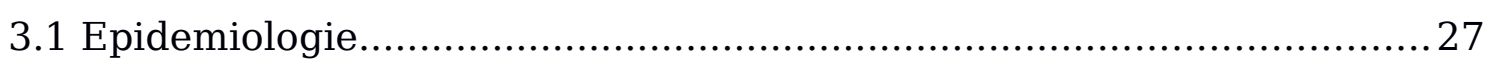

3.2 Lokalisation der Aneurysmen................................................. 28 
3.2.1 Lokalisation der für SAB ursächlichen Aneurysmen .28

3.2.2 Lokalisation der für SAB ursächlichen Aneurysmen mit späterem Vasospasmus. 29

3.3 Auswertbarkeit der DSA hinsichtlich Vasospasmus.......................... 30

3.4 Lokalisation der Vasospasmen...................................................... 31

3.5 Zeitliches Auftreten der Vasospasmen....................................... 31

3.6 Behandlung der SAB und vasospasmusbedingten Infarkte.................32

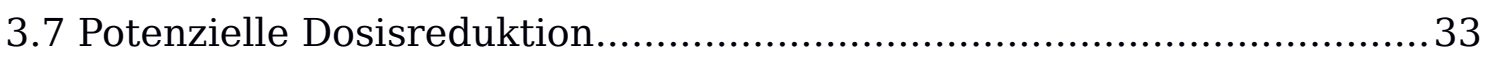

3.8 Subjektiver Eindruck der Bilddiagnostik................................... 34

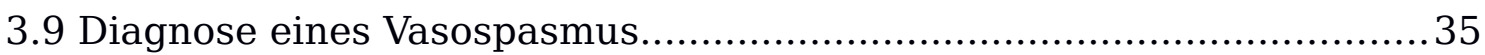

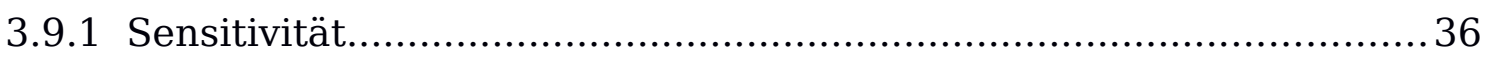

3.9 .2 Spezifität................................................................. 38

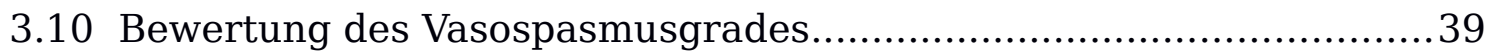

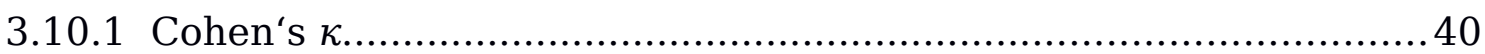

3.10.2 Pearson-Korrelation........................................................... 41

3.11 Interrater-Reliabilität der Vasospasmusdetektion..........................43

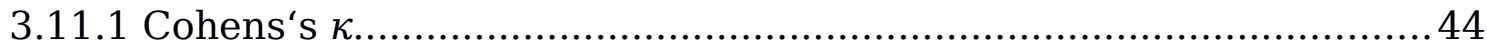

3.11.2 Pearson-Korrelation.......................................................... 46

3.12 Interrater-Reliabilität des Vasospasmusgrades.............................46

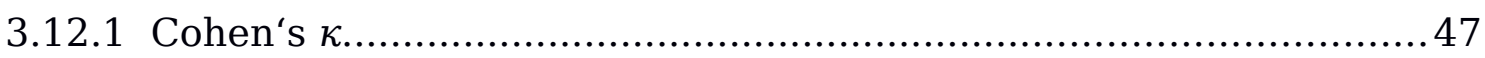

3.12.2 Pearson-Korrelation........................................................... 48

4 Diskussion.........................................................51

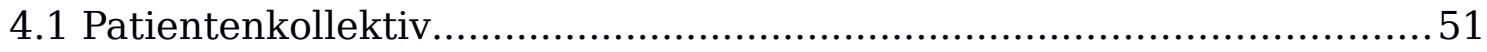

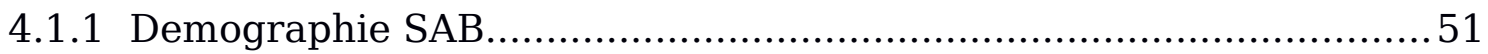

4.1.2 Vasospasmusinzidenz nach SAB........................................ 52

4.1.3 Lokalisation der für die SAB ursächlichen Aneurysma.....................52

4.1.4 Behandlungsmethode der SAB.............................................. 53

4.1.5 Vasospasmus und vasospasmusbedingte Infarkte..........................53

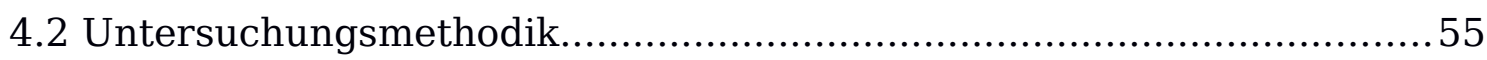

4.2.1 Diagnostischer Mehrwert durch 4D-CTA..................................56

4.3 Vergleich mit anderen Bildauswertungsprotokollen............................58

4.3.1 Einfluss des Rekonstruktionszeitpunktes................................58

4.3.2 Einfluss der Schichtdicke................................................. 58

4.3.3 Rekonstruktion mittels temporal maximum intensity projection.......59

4.4 Dosisreduktion.................................................................... 60

4.5 Datenlage für die hintere Zirkulation...................................... 61

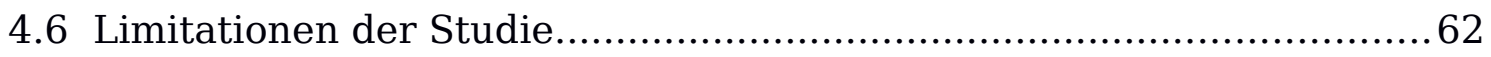

5 Zusammenfassung...................................................64

6 Referenzen......................................................................65 


\section{Abbildungsverzeichnis}

Abbildung 1: Auswahl der Patienten............................................

Abbildung 2: Kontrastmittelanreicherungskurve...........................15

Abbildung 3: Exemplarische Darstellungen der verschiedenen bildge-

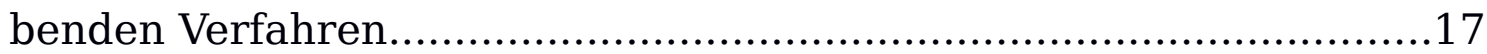

Abbildung 4: Gefäßeinteilung zur Bewertung..............................19

Abbildung 5: Epidemiologie der SAB und Vasospasmen.................28

Abbildung 6: Lokalisation der Aneurysmen..................................29

Abbildung 7: Auswertung der Vasospasmen (DSA).........................30

Abbildung 8: Zeitliches Auftreten von Vasospasmen........................31

Abbildung 9: Potenzielle Dosisreduktionen......................................33

Abbildung 10: Vergleich von Standard-MIP und früharterieller 4D-

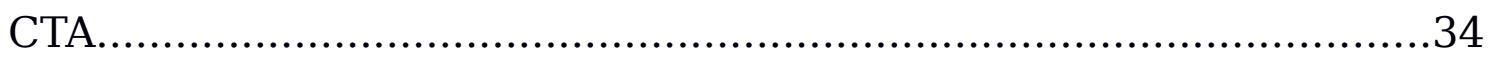

Abbildung 11: Sensitivität der Vasospasmusdetektion....................36

Abbildung 12: Spezifität der Vasospasmusdetektion........................38

Abbildung 13: Beurteilung des Vasospasmusgrades (Interrater-Reliabilität).

Abbildung 14: Beurteilung des Vasospasmusgrades (Pearson-Korrelation).

Abbildung 15: Interrater-Reliabilität der Vasospasmusdetektion.....44 Abbildung 16: Interrater-Korrelation der Vasospasmusdetektion.....45 Abbildung 17: Interrater-Relibilität der Beurteilung des Vasospasmusgrades zwischen Rater A und B 48

Abbildung 18: Korrelation der Beurteilung des Vasospasmusgrades zwischen Rater A und B 


\section{Tabellenverzeichnis}

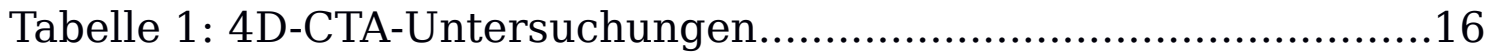

Tabelle 2: Schweregradeinteilung des Vasospasmus.........................20

Tabelle 3: Vierfeldertafel.............................................................24 


\section{Abkürzungsverzeichnis}

ACA

ACOM

AICA

BA

$\mathrm{CBF}$

CBV

cCT

CT

CTA

4D-CTA

CTDI

CTP

DCI

DIND

DLP

DNA

DSA

$\mathrm{HU}$

ICA

ISAT

(95\%-)KI

MCA

MDCT

MIP

MRT

$\mathrm{P}(\mathrm{C}) \mathrm{A}$

PCOM

PICA

SAB

SCA

SOP

TCCD

TCD

tMIP

TTP

UMG

VA
Arteria cerebri anterior

Arteria communicans anterior

Arteria inferior anterior cerebelli

Arteria basilaris

cerebral blood flow

cerebral blood volume

Kranielle CT

Computertomographie

Computertomographie-Angiographie

4D-Computertomographie-Angiographie computed tomography dose index

Perfusions-CT

delayed cerebral ischemia

delayed ischaemic neurological deficits

Dosislängenprodukt

Desoxyribonukleinsäure

Digitale Subtraktionsangiographie

Hounsfield-Unit

Arteria carotis interna

international subarachnoid aneurysm trial

(95\%-)Konfidenzintervall

Arteria cerebri media

Multidetektor-Computertomographen

maximum intensity projection

Magnetresonanztomographie

posterior (cerebral) artery

Arteria posterior communicans

posterior inferier cerebellar artery

Subarachnoidalblutung

Arteria superior cerebelli

standard operating procedure

transcranial colour-coded duplex sonography

Transkranielle Dopplersonographie

temporal maximum intensity projection

time to peak

Universitätsmedizin Göttingen

Arteria vertebralis 


\section{$1 \quad$ Einleitung}

Zerebrale Vasospasmen als eine der Hauptkomplikation von Subarachnoidalblutungen (SAB) stellen eine diagnostische und therapeutische Herausforderung dar. Insbesondere bei intubierten Patienten erfordern die begrenzten klinischen Untersuchungsmöglichkeiten und die unzureichende Aussagekraft der Doppler-Sonographie eine engmaschige Überwachung von Patienten mit bildgebenden Verfahren (Findlay et al. 2016).

SAB selbst sind ernste neurologische Notfälle und bedingen circa 5\% aller Schlaganfälle (van Gijn und Rinkel 2001). Bei einer SAB-Inzidenz von 6 - 9 / 100.000 in den USA und Zentraleuropa besteht eine Mortalität von 35\% bis zu 50\% nach 30 Tagen (da Costa et al. 2004; Feigin et al. 2015). Das Outcome einer SAB hängt neben dem Ausmaß der initialen Blutung und des therapeutischen Erfolgs der Aneurysmabehandlung maßgeblich von den im Verlauf auftretenden Komplikationen ab. Neben Rezidivblutungen, Elektrolytentgleisungen, kardialer Dekompensation und der Entwicklung eines Hydrocephalus (Graf 1971; van Gijn et al. 2007; Zhao et al. 2016) stellen zerebrale Ischämien im Verlauf eine der Hauptkomplikationen dar (Fisher et al. 1977; Heros et al. 1983; Boulouis et al. 2017). Diese sind eine der Hauptursachen für Morbidität and Mortalität und treten bei $30 \%$ bis $46 \%$ der Patienten auf, die die SAB initial überlebt haben (Vergouwen et al. 2010; Velat et al. 2011).

Wegen ihres verzögerten Auftretens werden diese zerebralen Ischämien Delayed Cerebral Ischemia (DCI) genannt, auch wenn andere Namen in der Literatur gebräuchlich sind, z. B. Delayed Ischaemic Neurological Deficits (DIND, Velat et al. 2011) oder Vasospasmus. Aufgrund dieser Vielfalt von Begriffen und der oft unklaren Vermischung von klinischen, angiographischen und bildmorphologischen Korrelaten, wird hier auf die Definition nach Vergouwen et al. (2010) zurückgegriffen: „Eine Verschlechterung des klinischen Zustands infolge einer DCI umfasst fokale neurologische Defizite und/oder eine Reduktion um mindestens zwei Punkte auf der Glasgow-Coma-Scale, die (1) mindestens eine Stunde andauern, (2) in zeitlichem Abstand 
zur SAB auftreten und (3) nicht durch andere Ursachen erklärt werden können." Hierbei handelt es sich also um eine Ausschlussdiagnose (Rowland et al. 2012). Neben den fokal-neurologischen Defiziten und der wechselhaften Bewusstseinseintrübung wurden auch Meningismus und progredienter Kopfschmerz als häufige klinische Symptome beschrieben (Fisher et al. 1977; Hijdra et al. 1986; Ferguson und Macdonald 2007; Mijiti et al. 2016). Demgegenüber steht die Definition des zerebralen Infarktes, der einen direkten Nachweis in Computertomographie (CT), Magnetresonanztomographie (MRT) oder Autopsie erfordert. Hiervon abzugrenzen ist weiterhin der Vasospasmus im engeren Sinne, der den angiographischen Nachweis einer Gefäßengstellung darstellt.

Die genaue Pathophysiologie von DCI ist nicht ausreichend geklärt und es kann zu einer Verschlechterung des Zustandes auch ohne bilddiagnostischen Nachweis eines Infarktes oder Vasospasmus kommen. So ließen sich zerebrale Infarkte bildmorphologisch (CT oder MRT) nur bei 10 - 27\% der Patienten mit klinischer Verschlechterung infolge von DCI darstellen (Lanzino et al. 1999; Macdonald et al. 2011). Pathogenetisch werden neben Vasospasmen weitere mögliche Ursachen für DCI diskutiert, zum Beispiel frühe Hirnschädigungen nach der SAB oder Mikrothrombosen (Rowland et al. 2012). Insbesondere führen Rowland et al. (2012) eine Spreading Depolarization des geschädigten Gewebes an. Dieses Phänomen beschreibt eine sich langsam ausbreitende Depolarisationswelle, die in geschädigtem Gewebe zu einer Ischämie führen kann. Trotz dieser multifaktoriellen Pathogenese scheinen Vasospasmen dennoch eine tragende Rolle zu spielen.

Das typische Auftreten von DCI infolge einer SAB bedingt diagnostische Herausforderungen, da Bewusstseinsveränderungen und -eintrübungen nach $\mathrm{SAB}$ auch durch andere Ursachen auftreten können. So kommt es bei $15 \%$ der Patienten mit SAB zu einer erneuten Blutung, 50\% entwickeln einen Hydrocephalus. Kardiovaskuläre Beeinträchtigungen und Elektrolytverschiebungen können ebenfalls zu Bewusstseinsbeeinträchtigungen führen (van Gijn und Rinkel 2001; Findlay et al. 2016). Diese alternativen Ursachen sind zum einen differentialdiagnostisch auszuschließen. Zum anderen können allein aufgrund der Bewusstseinseintrübung als Folge der SAB viele Patien- 
ten nicht adäquat körperlich und neurologisch untersucht werden. Daher bleiben Verschlechterungen des neurologischen Zustandes durch DCI, die typischerweise zwischen dem fünften und 14. Tag nach der SAB auftreten (Fisher et al. 1977; Heros et al. 1983), klinisch oft unbemerkt.

Klinische Symptome alleine sind kein verlässliches Diagnosekriterium für den Ausschluss einer DCI, wie die folgenden Studien verdeutlichen. Während sich bei 30\% - 50\% der Patienten infolge einer SAB eine DCI klinisch manifestiert, erleiden bis zu 70\% aller Patienten nach einer SAB einen angiographisch sichtbaren Vasospasmus (Fisher et al. 1977; Heros et al. 1983; Dorsch und King 1994; Velat et al. 2011; Findlay et al. 2016). Dabei entwickeln ca. 50\% der symptomatischen Patienten im Verlauf einen zerebralen Infarkt (Findlay et al. 2016). Vasospasmen sind für bis zu 10\% aller tödlichen Verläufe einer SAB verantwortlich (Dorsch und King 1994).

Dies verdeutlicht den Stellenwert einer schnellen Diagnostik und Behandlung von im Verlauf einer SAB auftretenden Vasospasmen, um die zum Teil gravierenden Folgen einer zerebralen Ischämie zu minimieren oder ganz zu verhindern. Dafür ist es erforderlich, dass eine Möglichkeit besteht, eine zuverlässige Diagnose ausschließlich mithilfe von bildgebenden Verfahren zu stellen. Zur Diagnostik eines Vasospasmus nach SAB stehen verschiedene bildgebende Verfahren zur Verfügung.

Hierfür ist zum einen ist die transkranielle Dopplersonographie ein etabliertes Verfahren. Die transkranielle Dopplersonographie macht sich zunutze, dass es durch die Verengung eines Gefäßes zum Anstieg der Geschwindigkeit des Blutflusses kommt. Dieser Anstieg kann durch die Doppler-Sonographie festgestellt werden. Bei der A. Cerebri media (MCA) gelten sowohl (1) eine mittlere Strömungsgeschwindigkeit über $200 \mathrm{~cm} / \mathrm{s}$ als auch (2) ein Anstieg der mittleren Strömungsgeschwindigkeitum mehr als $50 \mathrm{~cm} / \mathrm{s}$ in 24 Stunden oder aber auch (3) der hemispheric index über 3 als Vergleichsmaß extraund intrakranieller Geschwindigkeiten als Diagnosekriterien für einen Vasospasmus (Leitlinie Subarachnoidalblutung 2012). Hierbei handelt es sich also um einen nicht-invasiven, indirekten Nachweis eines Vasospasmus, der in der klinischen Praxis routinemäßig zur Detektion von Vasospasmen eingesetzt wird. Er kann zwar von erfah- 
renen Ärzten verlässlich für die Detektion von DCI der proximalen Gefäße des Circulus Wilisii angewendet werden (Findlay et al. 2016), weist aber einige Limitierungen auf. (1) Die Aussagekraft der TCD ist stark abhängig von der Expertise des behandelnden Arztes. (2) Sie ist nur verlässlich bei den proximalen Gefäßen des Circulus Wilisii, sie versagt jedoch bei den Gefäßen distal des Circulus Wilisii und bei niedrigen Strömungsgeschwindigkeiten (Leitlinie Subarachnoidalblutung 2012; Findlay et al. 2016). Dies liegt darin begründet, dass die MCA aufgrund ihres Verlaufes typischerweise in einem Winkel von weniger als $30^{\circ}$ zum Schallstrahl verläuft. Im Gegensatz hierzu beträgt der Beschallungswinkel in den basalen Hirnarterien oft über $30^{\circ}$, wodurch sich deutlich größere Fehler bei der Messung der Strömungsgeschwindigkeit ergeben (Zimmermann 2010; Widder und Hamann 2018). (3) Nicht bei allen Patienten ist ein für eine TCD ausreichendes Schallfenster gegeben. In bis zu 10\% der Fälle, bei älteren Frauen bis zu 30\%, ist das Schallfenster stark verkleinert oder sogar verschwunden. In diesen Fällen ist keine adäquate TCD-Diagnostik möglich (Seidel et al. 1995; Baumgartner et al. 1997a; Baumgartner et al. 1997b). Bei Verwendung einer Duplexsonographie (Transcranial color coded doppler, TCCD) kann zwar die Winkelabhängigkeit korrigiert werden, das Problem der Schallfenster bleibt aber auch hier (Purkayastha und Farzaneh 2014). Weder TCD noch TCCD sind also allgemein zuverlässig für die Diagnostik von DCI und sowohl ihr positiv prädiktiver Wert, als auch ihr negativ prädiktiver Wert werden als unzureichend erachtet (Lysakowski et al. 2001; Rabinstein et al. 2004).

Zum anderen wird zur Vasospasmusdiagnostik die Computertomographie-Angiographie (CTA) routinemäßig angewandt. Die Rekonstruktion von angiographischen Bildern durch die CT-basierte CTA ist in Abschnitt 2.2.2 ausführlich beschrieben. Errechnete angiographische Bilder aus einem CT-Scan nach venöser Kontrastmittelgabe (CTAngiographie, CTA) können bei ausreichender Erfahrung und Kenntnis des behandelnden Arztes Vasospasmen zuverlässig darstellen. Abgesehen von möglichen allergischen Reaktion auf das Kontrastmittel wird der Patient einer relativ hohen Strahlendosis ausgesetzt, auch wenn sich die Untersuchungszeit durch die Verwendung von Multidetektor-Computertomographen (MDCT) verkürzt hat. 
Die Standard-CTA wird zu einem fest definierten Zeitpunkt der Kontrastmittelanreichung in den zerebralen Gefäßen aufgenommen. Dieser Zeitpunkt soll für ein breites Patientenkollektiv eine ausreichende Kontrastmittelanreicherung in den kranialen Gefäßen selbst bei Patienten mit verringertem kardialem Auswurf sicherstellen. Dies ist sinnvoll für einen Gesamtüberblick der zerebralen Gefäße, für Malformationen und Aneurysmen (Pedersen et al. 2001; Agid et al. 2006) sowie für die Apoplex-Diagnostik (Knauth et al. 1997; Hennerici 2015). Für die Diagnostik der DCI ist der Nutzen des verwendeten Zeitpunktes der CTA zumindest fraglich. Die venöse Überlagerung könnte hierbei die Diagnostik erschweren. Für die CTA kann jedoch nicht einfach ein früherer Zeitpunkt der Kontrastmittelanreicherung ausgewählt werden, da unter Berücksichtigung der Verzögerung bei kardial vorbelasteten Patienten mit einer niedrigen Ejektionsfraktion die Gefäße schlechter dargestellt werden (Schregel et al. 2018).

Darüber hinaus ist die Perfusions-CT (CTP) als wichtiger Prädiktor für das Autreten von DIND etabliert (Malinova et al. 2020). Die Technik der CTP ist in Abschnitt 2.2.3 ausführlich erläutert. Für die CTP werden nach Verabreichung eines intravenösen Kontrastmittel-Bolus repetitive CTAs durchgeführt, sodass die CTP den Verlauf der Kontrastmittelanreicherung und -abflutung in den zerebralen Gefäßen und im Parenchym darstellt. Der erzeugte, zeitlich aufgelöste, dreidimensionale (4D-) Datensatz kann zur Berechnung angiographischer Informationen verwendet werden. Dies wird als 4D-CTA oder dynamische CTA bezeichnet (Yang et al. 2008; Salomon et al. 2009; Brouwer et al. 2010; Smit et al. 2012). Somit kann hier eine CTA zu einem beliebigen Zeitpunkt herangezogen werden. Die CTP findet in den vergangenen Jahren zunehmend Verwendung zur Detektion von Vasospasmen.

Durch die Verwendung von standardisierten Untersuchungsroutinen (Kombination von Klinik, TCD, CTA und CTP) und (präventiver) medikamentöser Therapie konnte das Mortalitätsrisiko und das Risiko einer dauerhaften Behinderung allein durch DCI in den letzten Jahren zwar auf unter $10 \%$ aller Patienten mit DCI gesenkt werden. Es bleibt aber eine der Hauptursachen für das schlechte Outcome nach einer aneurysmalen SAB (Findlay et al. 2016). 
An der Universitätsmedizin Göttingen (UMG) ist eine Standard Operating Procedure (SOP) zum Management von Patienten nach SAB etabliert („Management von Patienten nach Subarachnoidalblutung“). Initial bei Verdacht auf SAB erhalten die Patienten ein kranielles CT (cCT), eine CTA und CTP sowie eine Digitale Subtraktionsangiographie (DSA) zur genauen Darstellung des Aneurysmas. Abhängig vom Befund wird dann interdisziplinär entschieden, ob der Patient coil-embolisiert oder mittels mikrochirurgischem Clipping behandelt wird. Postinterventionell bzw. postoperativ erfolgt eine cCTKontrolle.

Im Verlauf erhält der Patient tägliche klinisch-neurologische Untersuchungen sowie eine tägliche TCD-Messung. Ist er klinisch nicht beurteilbar, erhält er an Tag drei und sieben jeweils ein cCT, eine CTA des Cerebrums und eine CTP-Untersuchung. Verschlechtert er sich klinisch, erhält er ebenfalls eine bildgebende Kontrolle mit cCT, CTA (nur des Cerebrums) und CTP. Ergeben sich in der täglichen TCDMessung Werte über $120 \mathrm{~cm} / \mathrm{s}$ oder $\Delta \mathrm{TCD}$ von mehr als $50 \mathrm{~cm} / \mathrm{s}$, erhält der Patient ebenfalls unmittelbar eine cCT, CTA (nur des Cerebrums) und CTP-Untersuchung. Sofern der Patient (1) klinisch-neurologisch untersuchbar ist und es zu keiner klinischen Verschlechterung kommt und (2) bei der TCD-Untersuchung Normwerte aufweist, erfolgt eine cCT-Kontrolle routinemäßig erst am zehnten Tag. Durch diese Vielzahl an radiologischen Untersuchungen bei klinisch nicht beurteilbaren Patienten ergibt sich kumulativ eine erhebliche Strahlenbelastung.

\subsection{Ziel dieser Arbeit}

In dieser Arbeit soll untersucht werden, ob das oben ausführlich erläuterte Prozedere verändert werden kann, sodass die 4D-CTA die konventionelle CTA komplett ersetzen könnte, um die Strahlenbelastung zu reduzieren. Die Verwendung der 4D-CTA zur Spasmusdiagnostik wäre theoretisch vorteilhaft, da neben der Strahlendosis auch die Menge des verwendeten Kontrastmittels und die zum Scannen benötigte Zeit gesenkt werden könnten. Die Hypothese ist hierbei, dass die Verwendung der 4D-CTA vorteilhaft ist, da sie eine früharterielle Darstellung der Gefäße erlaubt. Es wird erwartet, dass Va- 
sospasmen ohne die venöse Überlagerung, die bei der konventionellen CTA in Kauf genommen wird, besser dargestellt werden können. In anderem Kontext konnte bereits gezeigt werden, dass die 4D-CTA bei der Detektion von arteriellen Verschlüssen nach einem Schlaganfall eine ähnliche Sensitivität und Spezifität wie bei der konventionellen CTA erreicht (Smit et al. 2015). Dies gibt Anlass zur Hoffnung, dass die 4D-CTA auch bei Vasospasmen nach SAB die CTA ersetzen kann.

Damit auf die konventionelle CTA verzichtet werden kann, sollte die 4D-CTA eine mindestens gleichwertige diagnostische Aussagekraft, d. h. gleiche Sensitivität und Spezifität, erreichen. Diese Arbeit vergleicht die diagnostische Aussagekraft von 4D-CTA und konventioneller CTA zur Beurteilung von Vasospasmen nach SAB folgendermaßen: (1) Der Gefäßstatus wird auf Basis von DSA, 4D-CTA und konventioneller CTA bestimmt. (2) 4D-CTA und konventionelle CTA werden hinsichtlich der Sensitivität und Spezifität zur Vasospasmusdiagnose miteinander verglichen, wobei die Bewertung des Gefäßstatus auf Basis der DSA hier als Goldstandard herangezogen wird, gegen den der Gefäßstatus aus 4D-CTA und CTA verglichen werden. (3) 4DCTA und konventionelle CTA werden hinsichtlich der Beurteilung des Schweregrades von Vasospasmen miteinander verglichen. (4) 4DCTA und konventionelle CTA werden hinsichtlich der Übereinstimmung von Ratern mit und ohne langjährige diagnostische Erfahrung miteinander verglichen.

Diese Dissertation ist wie folgt gegliedert. In Kapitel 2 wird die Methodik ausführlich beschrieben. Dies schließt das Studiendesign und die Patientenauswahl, die Technik der verwendeten bildgebenden Verfahren, die Auswertung der Bildgebung sowie die statistische Auswertung zur Beantwortung der gestellten Fragen ein. Die gewonnen Ergebnisse werden in Kapitel 3 dargestellt. Das abschließende Kapitel 4 diskutiert und bewertet die Ergebnisse im Kontext der in dieser Studie gestellten Fragen und der wissenschaftlichen Literatur. 


\section{Methodik}

Die Fragestellung wurde mithilfe einer retrospektiven Studie untersucht. Diese Studie wurde von der Ethikkommission der UMG genehmigt (Referenznummer: DOK_167_2016). Es wurden Patienten mit $\mathrm{SAB}$ in Betracht gezogen, die postinterventionell nach der SOP an der UMG untersucht wurden (siehe Abschnitte 2.1, 2.2 und 2.3). Für diese Patienten wurden DSA, CTA, und 4D-CTA von zwei Ratern unabhängig voneinander und verblindet hinsichtlich auftretenden Vasospasmen bewertet (siehe Abschnitt 2.4). Diese Daten wurden statistisch ausgewertet (siehe Abschnitte 2.5 und 2.6). Alle Schritte sind im Folgenden detailliert erläutert.

\subsection{Patientenauswahl}

Alle Schritte der Patientenauswahl sind in Abbildung 1 schematisiert. Ausgewählt wurden alle Patienten, die in der Klinik für Neuroradiologie der Universitätsmedizin Göttingen in einem Zeitraum von Juli 2012 bis einschließlich Dezember 2016 mit Erstdiagnose SAB nach rupturiertem Aneurysma untersucht wurden (Kollektiv (i) in Abbildung 1). Dies waren insgesamt 115 Patienten. Von diesem Patientenkollektiv wurde im Rahmen dieser Dissertation eine Datenbank erstellt mit folgenden Parametern: Alter und Geschlecht des Patienten, Ausprägungsgrad der SAB nach Hunt und Hess (1968), Fisher-Score

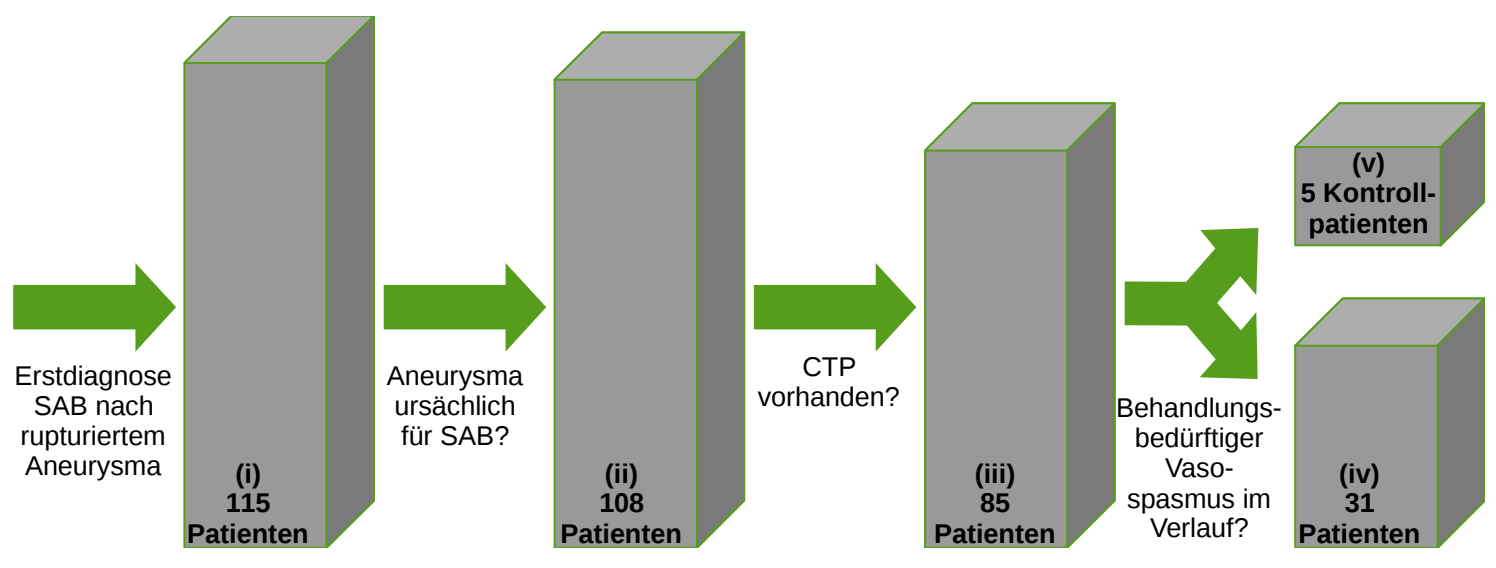

Abbildung 1: Auswahl der Patienten. Flussdiagramm zur Darstellung der Patientenauswahl. Details siehe Text. 
(Fisher et al. 1980), Aneurysma-Lokalisation, -anzahl, -größe und -behandlung, Infarktentwicklung und -lokalisation zu definierten Zeitpunkten im Verlauf der SAB, die Entwicklung eines interventionspflichtigen Vasospasmus und dessen Behandlung sowie die Entwicklung eines vasospasmusbedingten Infarktes. Als Infarkt wurde eine neu aufgetretene und persistierende Hypodensität im cCT gewertet (Yoshimoto et al. 1999). Bei einem vasospasmusbedingten Infarkt musste die Lokalisation des Infarktes passend zur Lokalisation des Vasospasmus und nicht durch andere Pathologien erklärbar sein.

Die Daten wurden in einer umfassenden Tabelle pseudonymisiert zusammengefasst. Die Erstellung der Datenbank wurde von einem radiologischen Facharzt mit der Zusatzbezeichnung Neuroradiologie kontrolliert.

Gemäß klinischem Protokoll waren von diesem Patientenkollektiv grundsätzlich computertomographische Aufnahmebefunde vorhanden. Falls hierbei keine SAB oder kein für die SAB ursächliches Aneurysma bestätigt werden konnte, wurden die entsprechenden Patienten ausgeschlossen. Dies war bei sieben Patienten der Fall (drei Patienten ohne SAB, vier mit SAB ohne Aneurysma). Von den verbleibenden 108 Patienten (Kollektiv (ii) in Abbildung 1) wurden grundsätzlich Verlaufskontrollen mittels CT nach dem in der Einleitung detailliertem Protokoll durchgeführt. Es kamen jedoch nur diejenigen Patienten infrage und wurden für die Studie herangezogen, bei denen auch ein CTP-Datensatz vorhanden war (Kollektiv (iii) in Abbildung 1). Dies war nicht der Fall, falls Patienten im Verlauf klinisch beurteilbar wurden und daher nicht weiter nach dem SOP behandelt wurden. Daher wurden 23 Patienten ausgeschlossen. Zusätzlich zum CT wurden auch CTA-Bildgebungen zur Kontrolle durchgeführt. Bei allen 85 ausgewählten Patienten waren daher sowohl CTA- als auch CTP-Untersuchungen vorhanden.

Von diesem Patientenkollektiv von 85 Patienten entwickelten 31 im Verlauf einen behandlungsbedürftigen Vasospasmus, der eine Intervention nötig machte (Kollektiv (iv) in Abbildung 1). Von diesen Patienten lagen daher auch Ergebnisse einer DSA-Untersuchung zum Zeitpunkt der Intervention vor. Diese wurden als Goldstandard zur Bewertung des Schweregrades des Vasospasmus herangezogen (siehe Abschnitt 2.4). Zusätzlich wurden fünf zufällig ausgewählte Pati- 
enten ohne interventionell behandlungsbedürftigen Vasospasmus, also auch ohne durchgeführte DSA als Goldstandard, als Kontrollgruppe berücksichtigt (Kollektiv (v) in Abbildung 1).

Zusammenfassend wurden in dieser Studie daher die Daten von 31 Patienten mit interventionell behandlungsbedürftigen Vasospasmen betrachtet, von denen jeweils CTA, CTP und DSA zur Verfügung standen. Zusätzlich wurden fünf Patienten ohne interventionell behandlungsbedürftigen Vasospasmus als Kontrollgruppe untersucht, für die nur CTA und CTP zur Verfügung standen, jedoch keine DSA.

\subsection{Technik der bildgebenden Verfahren}

Für diese Studie wurden Daten von CTA, aus CTP rekonstruierter 4D-CTA sowie DSA untersucht.

\subsubsection{DSA}

Als Goldstandard der Vasospasmusdiagnostik wurde die DSA verwendet. Hierbei wird die betrachtete zerebrale Region zunächst als Leeraufnahme ohne Kontrastmittel röntgenologisch dargestellt. Dies wird als Maske bezeichnet. Im weiteren Verlauf der Untersuchung wird wie auch bei der CTA Kontrastmittel (meist in die A. Brachialis oder A. Femoralis) injiziert, welches sich in den zerebralen Gefäßen anreichert. Es erfolgen nun weitere Aufnahme derselben Region, nur dass jetzt durch die Kontrastmittelgabe zusätzlich die Gefäße röntgenologisch dargestellt werden. Mittels der Subtraktionstechnik wird von der Maske die Aufnahme mit Kontrastmittelgabe abgezogen. Herausgerechnet werden also alle Elemente, die in beiden Aufnahmen vorhanden sind. Übrig bleiben die veränderten Bildanteile (Kahl-Scholz und Vockelmann 2017, S. 36). Als Ergebnis erhält man eine exakte und ausschließliche Darstellung der Gefäße und somit auch von Aneurysmen und Vasospasmen. Darstellbar sind Gefäße ab einem Durchmesser von $500 \mu \mathrm{m}$ (Anxionnat et al. 1998). Durch den kontinuierlichen Weiterfluss des Kontrastmittels im Gefäß und die Verdünnung des Kontrastmittels im Blut ist während der Serienaufnahme nur ein begrenzter Gefäßabschnitt darstellbar. Zur Darstellung des Gefäßes in ganzer Länge werden die Subtraktionsbilder aneinandergereiht, was als Summationsbild bezeichnet wird. Zur besseren Ori- 
entierung bei Interventionen kann dieses Summationsbild halbtransparent auf das Durchleuchtungsbild gelegt werden. Auch ohne weitere Kontrastmittelgabe ist so der Gefäßverlauf für den Untersucher ersichtlich (Kahl-Scholz und Vockelmann 2017, S. 37).

\subsubsection{CTA}

Bei einer CT-Untersuchung werden röntgenologisch transversale Schichtbilder erzeugt. Hierbei wird von einer um die Längsachse des Patienten rotierenden Röntgenröhre bzw. Detektor die winkelabhängige Schwächung der Röntgenstrahlung beim Durchtritt durch den Patienten gemessen. Diese wird zur Vergleichbarkeit in einer geeichten Dichteskala, der Hounsfield-Skala (Hounsfield Unit, HU), angegeben. Aus der winkelabhängigen Schwächung können durch mathematische Verfahren (Computed Tomography) Gewebeeigenschaften in der betrachteten Schicht rekonstruiert werden. Durch Vorschieben der Röntgenröhre bzw. des Detektors entlang der Längsachse des Patienten kann so eine überlagerungsfreie Erfassung eines Volumendatensatzes erreicht werden (Reiser et al. 2017, S. 85 - 87). Das dreidimensionale Korrelat eines zweidimensionales Bildelement ( $P i$ xel) nennt man hierbei Volumenelement (Voxel; Reiser et al. 2017, S. 85 - 90). Es gibt die räumliche Auflösung der CT an und errechnet sich aus Pixelgröße und Schichtdicke. Die dreidimensionale Darstellung unterscheidet die CT vom konventionellen Röntgen, bei dem das dreidimensionale Untersuchungsgebiet auf ein zweidimensionales Bild projiziert wird, und damit zwangsläufig alle Gewebeteile entlang des Strahlengangs überlagert.

Die ursprünglich sehr lange Dauer von CT-Untersuchungen konnte durch technische Fortschritte immer weiter gesenkt werden. So besitzen Multidetector-CT-Geräte (MDCT) im Gegensatz zu herkömmlichen CT-Geräten mehrere Detektoren nebeneinander, was eine kürzere Untersuchungszeit und größere Untersuchungsvolumina ermöglicht. Die Breite der einzelnen Detektoren (z. B. 0,5 mm bei einem 64-Zeilen-Scanner) ist ein Maß für die minimale Schichtdicke und somit die Ortsauflösung (Reiser et al. 2017, S. 87). Eine weitere Reduktion der Aufnahmezeit wurde durch den Übergang von der schichtweisen Aufnahme zur sogenannten Spiraltechnik mit kontinuierlicher Aufnahme und Tischvorschub erreicht. 
Die durch diese Fortschritte erzielte kürzere Untersuchungszeit und hohe örtliche Auflösung haben die Nutzung der CT für die angiographische Darstellung vieler Krankheitsbilder ermöglicht. Bei der CTAngiographie (CTA) wird intravenös jodhaltiges Kontrastmittel injiziert. Dies führt zu einer Dichtanhebung der Gefäße. Durch die kürzere Untersuchungszeit und hohe Ortsauflösung kann bei der CTA die arterielle Bolusphase erfasst werden. Der damit verbundene diagnostische Mehrwert hat dazu geführt, dass die CTA bei Krankheitsbildern wie Apoplex und SAB als Standard etabliert wurde (Reiser et al. 2017, S. 420).

Der Beginn der CTA-Untersuchung erfolgt automatisch nach dem Prinzip der Bolustriggerung. Auf einer gewählten Schichtposition findet kontinuierlich eine Messung der Kontrastmittelkonzentration im definierten Gefäßlumen statt. Sobald eine vorher festgelegte HU (also Dichte) erreicht wird, startet das Gerät automatisch (KahlScholz und Vockelmann 2017, S. 44).

Bei dem Patientenkollektiv, das im Rahmen dieser Promotion untersucht worden ist, wurde grundsätzlich eine Maximum Intensity Projektion (MIP) durch das Programm syngo Multi Modality Workplace $2008 B$ generiert. Hierbei werden entlang der Projektionsrichtung die Strukturen mit der höchsten Dichte ermittelt und verstärkt dargestellt (Kahl-Scholz und Vockelmann 2017, S. 49). Für die in dieser Arbeit betrachteten axialen Darstellungen heißt dies, das jedes Pixel des MIP-Bildes das Maximum aller Schichten an der entsprechenden Position darstellt. Da die kontrastmittelangereicherten Gefäße typischerweise röntgendichter als das umliegende Gewebe sind, ermöglicht die MIP eine bessere Darstellung des Gefäßstatus und wird standardmäßig herangezogen (Kortman et al. 2015).

\subsubsection{CTP und 4D-CTA aus CTP}

Bei der CTP werden nach intravenöser Kontrastmittelgabe repetitiv CTA-Untersuchungen durchgeführt. Durch die hohe örtliche Auflösung und Verkürzung der Untersuchungszeit seit Verwendung der Spiraltechnik und der MDCT kann der first pass eines Kontrastmittelbolus durch diese Untersuchung dargestellt werden. Konkreter heißt dies: Dargestellt wird die Kontrastmittelan- und abflutung im Gehirn durch Änderung der Röntgendichte in den Gefäßen, also die arteriel- 
le Anflutung bis zur venösen Abflutung. Aus diesem Datensatz von zeitabhängigen CTAs werden farblich kodierte Karten über Perfusionsparameter vom Programm syngo Multi Modality Workplace $2008 B$ erstellt und können Rückschlüsse über die Durchblutung einer betreffenden Region gezogen werden.

Aus der Zeit-Dichte-Kurve können diagnostisch relevante Parameter errechnet werden, z. B. cerebral blood flow (CBF) und cerebral blood volume (CBV) und time to peak (TTP). Während CBF und CBV im Rahmen dieser Promotion nicht zu Rate gezogen wurden, ist die TTP für diese Studie relevant: Die TTP charakterisiert den zeitlichen Abstand zwischen Beginn der Kontrastmittelinjektion und der maximalen Anreicherung des Kontrastmittel im untersuchten Gewebe (Reichenbach et al. 1999).

Der zeitlich aufgelöste, dreidimensionale (4D-) Datensatz kann zur Berechnung angiographischer Informationen verwendet werden. Dies wird als 4D-CTA oder dynamische CTA bezeichnet (Yang et al. 2008; Salomon et al. 2009; Brouwer et al. 2010; Smit et al. 2012). Somit kann hier eine CTA zu einem beliebigen Zeitpunkt herangezogen werden.

\subsubsection{Geräte und Untersuchungsparameter}

Die DSA-Untersuchungen der im Rahmen des Patientenkollektivs betrachteten Patienten erfolgte mit dem Artis Q Siemens.

Bei allen Patienten des betrachteten Patientenkollektivs wurden die CT-Untersuchungen mit dem Gerät Somatom Definition AS durchgeführt. Die Rekonstruktion der CTA und der MIP-CTA erfolgte automatisch durch das Programm syngo Multi Modality Workplace $2008 B$. Für die manuelle Auswahl und Rekonstruktion der MIP aus dem 4DCTA-Datensatz wurde ebenfalls die Workstation syngo Multi Modality Workplace 2008B sowie die Softwareapplikation Volume Perfusion-CT Neuro von Siemens verwendet. Das verwendete Bildbearbeitungsprogramm war Centricity* PACS Radiology RA1000 Workstation. Mittels manueller Bildbearbeitung wurde eine möglichst orbitomeatale, achsparallele Rekonstruktion des Circulus Wilisii angestrebt. 
Für die CTP-Untersuchung erfolgten 30 konsekutive Spiral-CTs des Gehirns mit den folgenden Parametern: Höhe des Scanvolumens 96 $\mathrm{mm}$ entlang der Longitudinalachse, $2 \mathrm{~s}$ Verzögerung nach Kontrastmittelinjektion, mittlere zeitliche Auflösung 1,5 s (mittlere Zeit zwischen zwei Scans), Gesamtdauer $45 \mathrm{~s}$, Röhrenspannung 80 kV, Ladungsmenge $200 \mathrm{mAs}$, Umlaufzeit 0,3 s, Pitchfaktor 0,5, Kollimation $2 \times 64 \times 0,6 \mathrm{~mm}$, Schichtdicke 1,5 mm, Inkrement $1 \mathrm{~mm}$ (Kernel H20f, 512 Matrix). Intravenös appliziert wurde ein 36 mL Kontrastmittelbolus (Imeron 400; Bracco, Konstanz, Deutschland) mit einer Flussrate von $6 \mathrm{~mL} / \mathrm{s}$. Anschließend wurden $30 \mathrm{~mL} \mathrm{NaCl}$ mit einer Flussrate von $6 \mathrm{~mL} / \mathrm{s}$ appliziert.

Für die CTA-Untersuchung wurde ein Spiral-CT des Gehirns mit den folgenden Parametern durchgeführt: Röhrenspannung $120 \mathrm{kV}$, Ladungsmenge $120 \mathrm{mAs}$, Umlaufzeit 0,3 s, Pitchfaktor 0,6, Kollimation $2 \times 64 \times 0,6 \mathrm{~mm}$, Schichtdicke 0,75 mm, Inkrement 0,4 mm. Intravenös appliziert wurde ein $65 \mathrm{~mL}$ Kontrastmittelbolus (Imeron 400; Bracco, Konstanz) mit einem biphasischen Protokoll $(45 \mathrm{~mL}$ mit 6 $\mathrm{mL} / \mathrm{s}$, dann $15 \mathrm{~mL}$ mit $3 \mathrm{~mL} / \mathrm{s}$ ). Anschließend wurden $30 \mathrm{~mL} \mathrm{NaCl}$ mit einer Flussrate von $3 \mathrm{~mL} / \mathrm{s}$ appliziert. Die CTA wurde mittels BolusTriggerung im Aortenbogen (100 HU, 2 s Latenz) automatisiert gestartet.

Die effektive Strahlendosis, berechnet aus Dosislängenprodukt und Dosiskonversionsfaktor betrug 5,3 mSv für die CTP sowie 1,1 mSv für kraniozervicale CTA (mehr als $20 \mathrm{~cm}$ Entfernung vom Vertex) bzw. 2,1 mSv für die übrige kaudale CTA (mehr als $15 \mathrm{~cm}$ Entfernung vom Vertex, Frölich et al. 2013).

Für die 4D-CTA-Bildgebung wurde ein kommerziell vertriebenes Software-Paket benutzt (Dynamic Angio; Siemens Healthcare), das eine automatische Bewegungskorrektur und eine Rauschunterdrückung beinhaltet. Perfusion parameter maps wurden mit der $5 \mathrm{~mm}$ VPCT-Rekonstruktion (Neuro Perfusion CT; Siemens Healthcare) errechnet.

\subsection{Auswertung der Bildgebung}

Für alle Patienten des SAB-Patientenkollektivs wurde die Bildgebung von CCT, CTA und CTP aller drei Kontrolluntersuchungen an Tag drei, 
sieben und zehn ausgewertet, zusätzlich die DSA (sofern vorhanden). Für die Vasospasmusbewertung wurde von den drei Kontrolluntersuchungen im weiteren für die statistische Auswertung diejenige herangezogen, die der Intervention zeitlich am nächsten lag. Bei den Kontrollpatienten ohne Intervention wurden die Bildgebungen der zweiten Kontrolluntersuchung herangezogen.

Die zu untersuchenden CTP-Datensätze wurden zunächst in die Workstation syngo Multi Modality Workplace 2008B eingeladen. Anhand der Zeit-Dichte-Kurve wurde für jeden Patienten individuell der Zeitpunkt der zu untersuchenden früh- und der spätarteriellen Phase bestimmt (Abbildung 2): zum einen der Zeitpunkt der maximalen arteriellen Kontrastmittelkonzentration (spätarterielle Darstellung, Maximum der Kontrastmittelanreicherungskurve), zum anderen der Zeitpunkt der maximalen Zunahme der arteriellen Kontrastmittelkonzentration (früharterielle Darstellung, maximale Steigung der Kontrastmittelanreicherungskurve, TTP). Die zu diesen Zeitpunkten jeweils erfolgte CTA-Serie wurde identifiziert. Nach Bewegungskorrek-

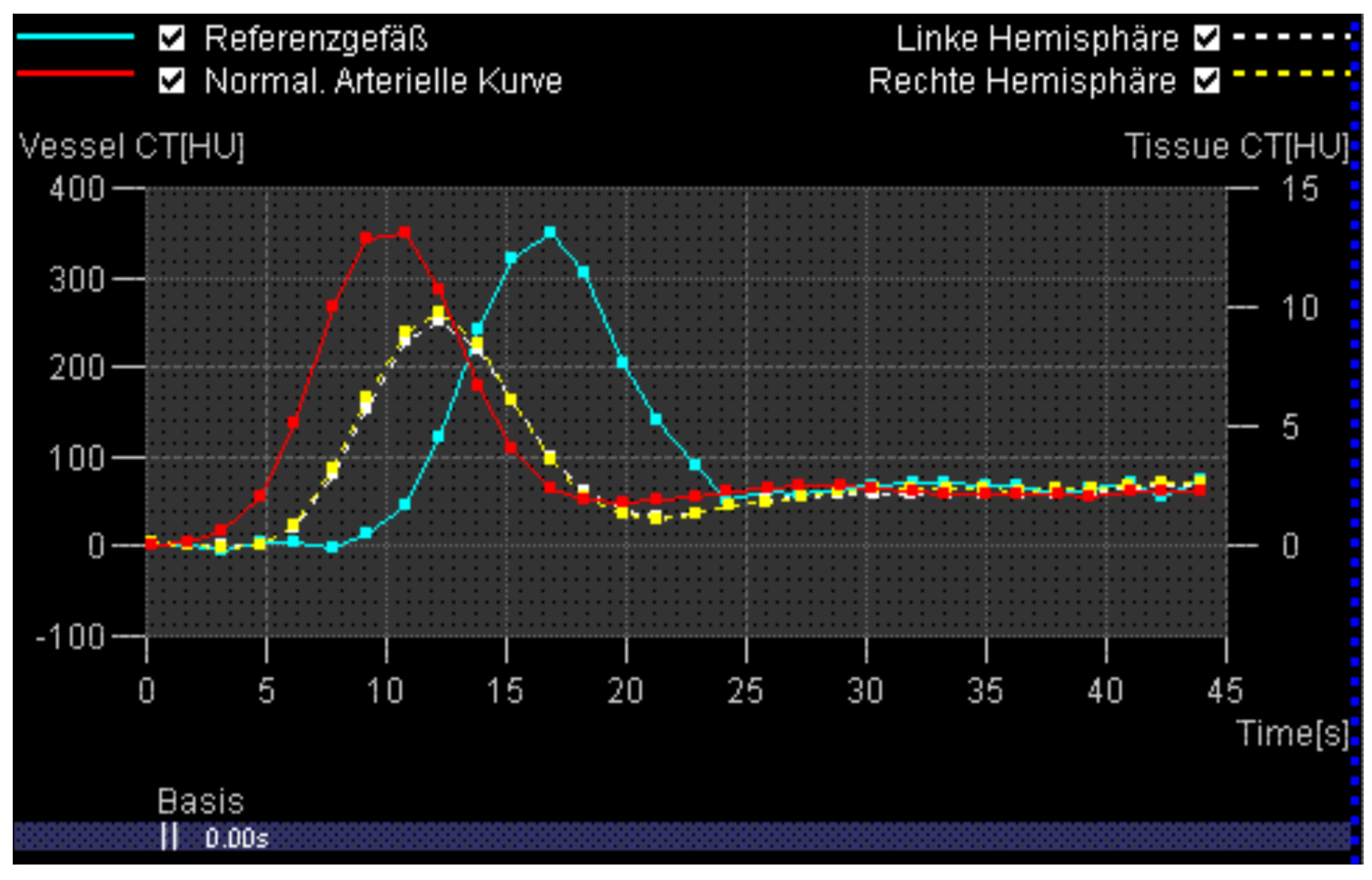

Abbildung 2: Kontrastmittelanreicherungskurve. Der zeitliche Verlauf der Abschwächung der Röntgenstrahlung in den Gefäßen ergibt die Kontrastmittelanreicherungskurve für venöse (blau) und arterielle (rot) Gefäße. 
tur und Segmentierung sowie manueller Korrektur (siehe oben) wurde mit der Workstation hieraus je eine MIP rekonstruiert. Aus dem MIP-Datensatz wurde zur Vergleichbarkeit der Verfahren ein singuläres Bild ausgewählt, das alle zu untersuchenden Gefäße bestmöglich darstellt.

Hierbei wurden zwei verschiedene MIP-Schichtdicken (5 mm und 10 $\mathrm{mm}$ ) und zwei verschiedene Zeitpunkte der Perfusion herangezogen. Somit ergeben sich insgesamt vier verschiedene Kombinationsmöglichkeiten, sodass final vier verschiedene rekonstruierte 4D-CTA-Bildgebungen untereinander und mit der CTA verglichen wurden (Tabelle 1): (1) Früharteriell (maximale Zunahme der Kontrastmittelkonzentration) mit $5 \mathrm{~mm}$ MIP-Schichtdicke (4D-CTA $\mathrm{fa}_{\mathrm{f}, 5 \mathrm{~mm}}$, Abbildung 3 Mitte links), (2) früharteriell mit $10 \mathrm{~mm}$ MIP-Schichtdicke (4D$\mathrm{CTA}_{\mathrm{fa}, 10 \mathrm{~mm}}$, Abbildung 3 Mitte rechts), (3) spätarteriell (maximale Kontrastmittelkonzentration) mit $5 \mathrm{~mm}$ MIP-Schichtdicke (4D-CTA ${ }_{\mathrm{sa}, 5 \mathrm{~mm}}$, Abbildung 3 unten links), (4) spätarteriell mit $10 \mathrm{~mm}$ MIP-Schichtdicke (4D-CTA sa, $10 \mathrm{~mm}_{\text {, }}$ Abbildung 3 unten rechts).

Tabelle 1: 4D-CTA-Untersuchungen.

Zeitpunkt

MIP-Schichtdicke

$5 \mathrm{~mm}$

$10 \mathrm{~mm}$

\begin{tabular}{cc}
\multicolumn{2}{c}{ Zeitpunkt } \\
\hline früharteriell & spätarteriell \\
\hline $4 \mathrm{D}-\mathrm{CTA}_{\mathrm{fa}, 5 \mathrm{~mm}}$ & $4 \mathrm{D}-\mathrm{CTA}_{\mathrm{sa}, 5 \mathrm{~mm}}$ \\
$4 \mathrm{D}-\mathrm{CTA}_{\mathrm{fa}, 10 \mathrm{~mm}}$ & $\mathrm{CTA}_{\mathrm{sa}, 10 \mathrm{~mm}}$
\end{tabular}

In allen Fällen wurde letztendlich die MIP einer CTA Bildgebung aus dem CTP-Datensatz ausgewertet. Zur Unterscheidung von der direkten CTA wird im folgenden von 4D-CTA gesprochen bzw. 4D-CTAx, wobei $\mathrm{X}$ ein beliebiges der oben genannten Verfahren darstellt. Entsprechend der direkten CTA wurde auch hier diejenige CTP für die Rekonstruktion ausgewählt, die der Intervention zeitlich am nächsten lag. Bei den Kontrollpatienten ohne Intervention wurde die CTP der zweiten Kontrolluntersuchung herangezogen. 
Die vorhandenen cCT-, CTA- und DSA-Aufnahmen aus der Routinediagnostik konnten direkt verwendet werden (Abbildung 3 oben links).
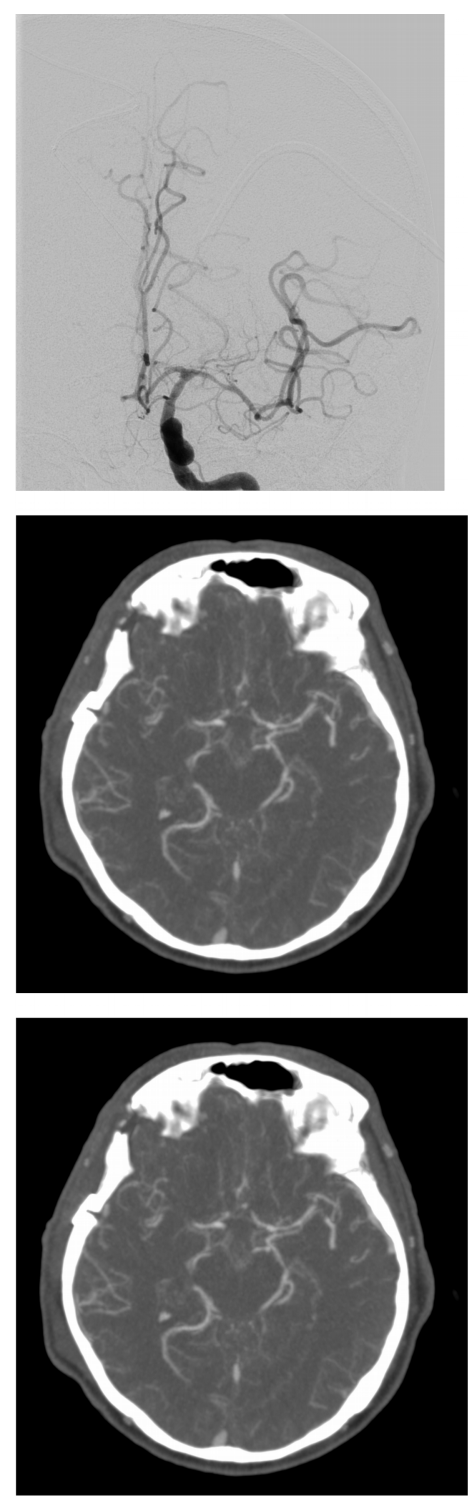
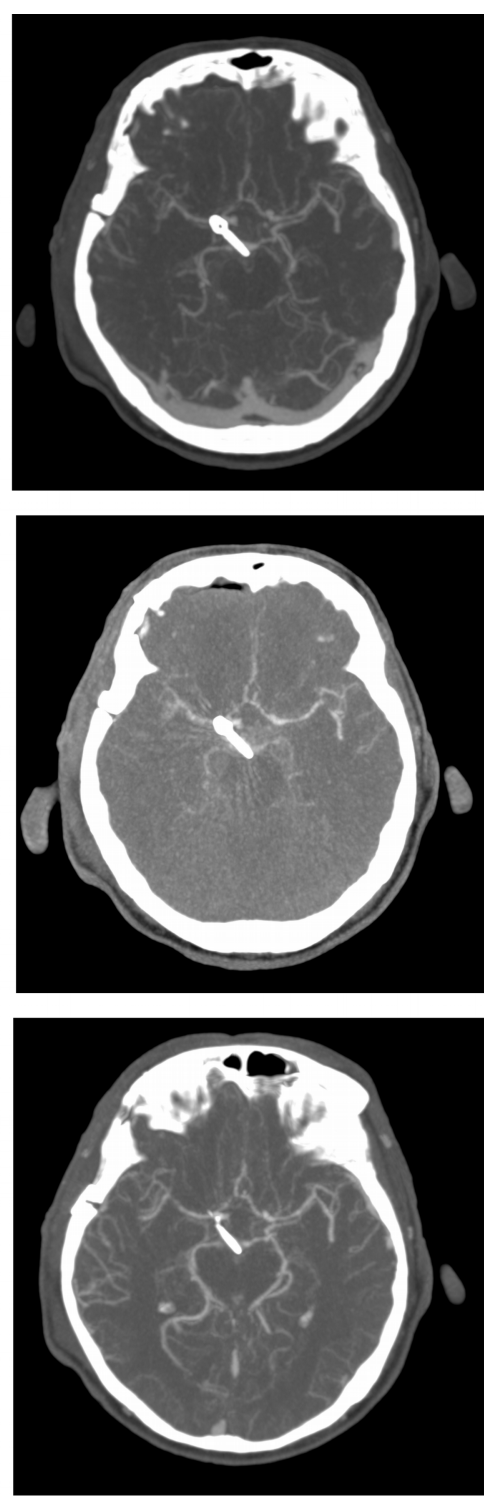

Abbildung 3: Exemplarische Darstellungen der verschiedenen bildgebenden Verfahren. Die Darstellungen sind gezeigt für einen 70-jährigen Patienten mit Vasospasmen im Bereich der linken ICA, MCA und ACA sowie der rechten MCA und ACA nach SAB bei rupturiertem und geclipptem Basilaris-Aneurysma. Oben links: DSA. Oben rechts: Standard-MIP aus CTA. Mitte links: MIP mit $5 \mathrm{~mm}$ Schichtdicke aus früharterieller Darstellung der 4D-CTA. Mitte rechts: MIP mit $10 \mathrm{~mm}$ Schichtdicke aus früharterieller Darstellung der 4D-CTA. Unten links: MIP mit $5 \mathrm{~mm}$ Schichtdicke aus spätarterieller Darstellung der 4D-CTA. Unten rechts: MIP mit $10 \mathrm{~mm}$ Schichtdicke aus spätarterieller Darstellung der 4D-CTA. 
Zur Vasospasmusdetektion wurde dabei die Maximum-IntensitätsProjektion (MIP, Abbildung 3 oben rechts) herangezogen.

\subsection{Rating des Gefäßstatus}

Die vorhandenen Bilder von CTA und 4D-CTA wurden von zwei Ratern unabhängig untersucht: ein erfahrener Assistenzarzt mit fünf Jahren Berufserfahrung in der Neuroradiologie sowie einer unerfahrenen Studierenden vor Beginn des praktischen Jahres. Die DSA als Goldstandard wurde von einem Facharzt der Radiologie mit neuroradiologischer Berufserfahrung ausgewertet. Da CTA und CTP an den jeweiligen Kontrolltagen (sowie die DSA bei nötiger Intervention) unmittelbar aufeinander folgend durchgeführt wurden, lagen sie in so engem zeitlichen Abstand zueinander, dass zwischen den Untersuchungen von einem fast identischen Gefäßstatus ausgegangen werden kann.

\subsubsection{Bewertete Gefäße}

Der Gefäßstatus wurde systematisch für neun verschiedene Arterien (oder Segmente von Arterien) des Circulus Wilisii untersucht (Abbildung 4): Terminale Arteria carotis interna (internal carotid artery, ICA) links und rechts, Arteria cerebri media (middle cerebral artery, MCA) links und rechts, Arteria cerebri anterior (anterior cerebral artery, ACA) links und rechts, Arteria cerebri posterior (posterior cerebral artery, PA) links und rechts, Arteria basilaris (basilar artery, BA). Die genannten Abkürzungen sowie die farbliche Codierung in Abbildung 4 werden im Folgenden beibehalten.

Abgesehen von der BA wurden alle Arterien getrennt für linke und rechte Hemisphäre untersucht. In einigen Fällen konnten Arterien aufgrund von starken Blutungen oder Artefakten in der Bildgebung nicht beurteilt werden. Die entsprechenden Arterien wurden dann von der weiteren Analyse ausgeschlossen.

\subsubsection{Ratingskala}

Für sämtliche Arterien wurde der Gefäßstatus anhand einer dreiwertigen Ordinalskala optisch bewertet. Wie von Haley Jr et al. (1993) und Elsayed et al. (2006) beschrieben, wurde hierbei der Schwere- 
grad des Vasospasmus wie in Tabelle 2 beschrieben beurteilt. Abgesehen hiervon waren einige Gefäße nicht bewertbar, wurden entsprechend markiert und nicht analysiert.

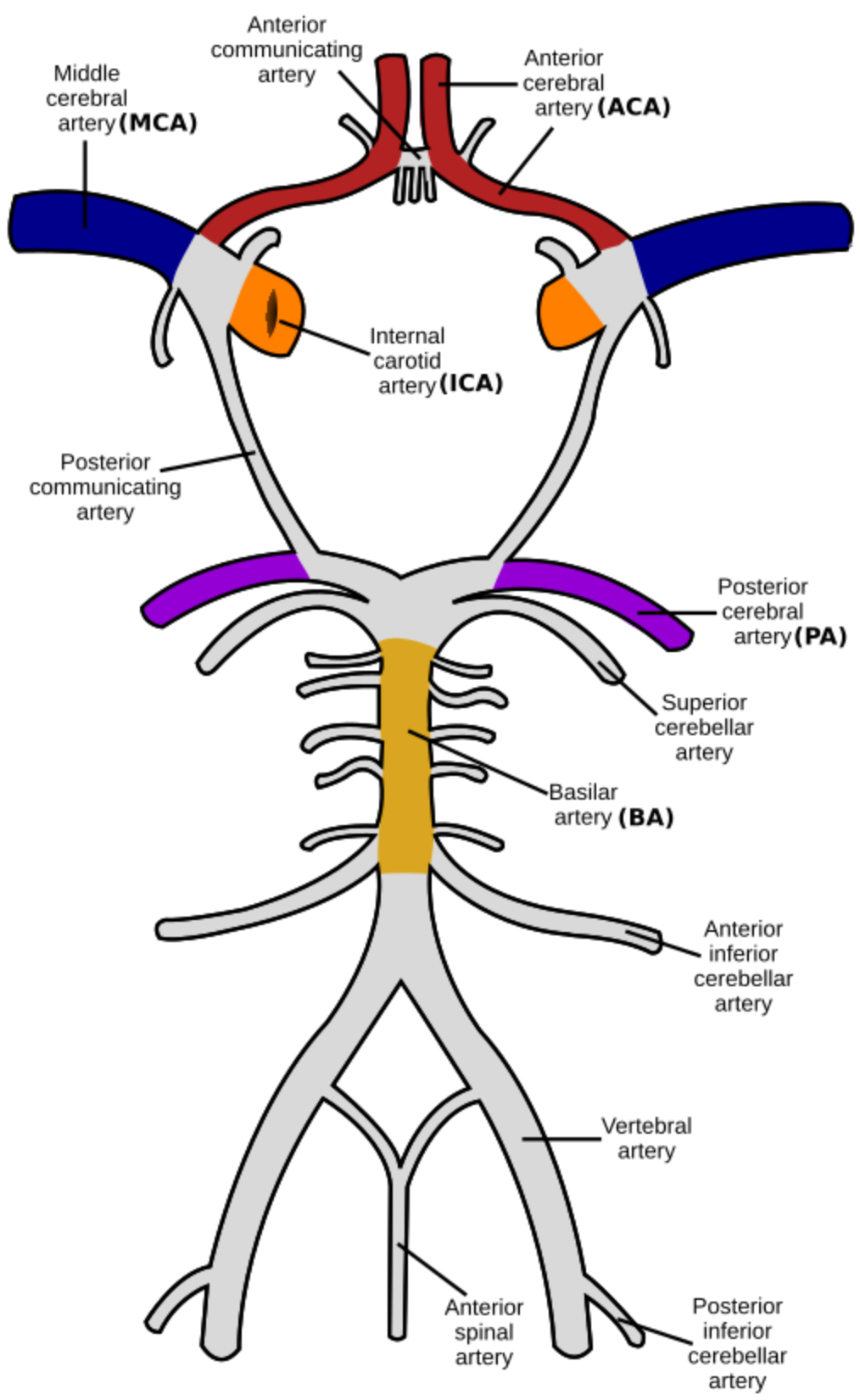

Abbildung 4: Gefäßeinteilung zur Bewertung. Es wurden neun verschiedene Gefäße beurteilt: Jeweils linke und rechte ACA (rot), MCA (blau), ICA (orange) und PA (violett), sowie die BA (gelb). Der Farbcode in allen folgenden Abbildung entspricht der hier getroffenen Konvention. Gemeinfreie Grafik modifiziert nach Gray (1918). 


\section{Tabelle 2: Schweregradeinteilung des Vasospasmus}

\section{Gefäßverengung}

(bezogen auf den Durchmesser)

weniger als $25 \%$ auf der gesamten sichtbaren Länge des Gefäßes

$25 \%$ bis $50 \%$ auf einem beliebig kurzen Abschnitt des Gefäßes

mehr als 50\% auf einem beliebig kurzen Abschnitt des Gefäßes

\section{Schweregrad}

nach Haley Jr et al. (1993) und

Elsayed et al. (2006)

1

kein relevanter Vasospasmus mäßig ausgeprägter 2

Vasospasmus

schwer ausgeprägter

Vasospasmus

\subsubsection{Unabhängige Auswertung}

Der Gefäßstatus wurde mit verblindeten Patientendaten erhoben. In jeweils einer Sitzung wurden einem Rater die Bilder eines Verfahrens (DSA, CTA, oder eine der vier verschiedenen 4D-CTA-Rekonstruktionen gemäß Abschnitt 2.2) präsentiert. Die Reihenfolge der Patienten war hierbei zufällig gewählt und von Sitzung zu Sitzung verschieden. Zwischen verschiedenen Sitzungen desselben Raters lagen mehrere Monate. Jeder Rater bewertete alle Gefäße (siehe Abschnitt 2.4.1) gemäß der festgelegten Skala (siehe Abschnitt 2.4.2). Die Protokollierung der Bewertung erfolgte hierbei jeweils nicht durch den bewertenden Rater, sondern durch eine unabhängige Person.

\subsection{Statistische Auswertung}

Nach der Auswertung der bildgebenden Diagnostik lagen folgende Ratings vor: für jeden Patient wurden jeweils linke und rechte ICA, MCA, ACA und PA sowie die BA bewertet. Ausgenommen von den Patienten der Kontrollgruppe war prinzipiell für jedes dieser Gefäße ein Rating aus der DSA vorhanden, welches als Goldstandard herangezo- 
gen wurde. Falls einzelne Gefäße in der DSA nicht dargestellt worden waren, wurden die Ratings mit allen Methoden für diese Gefäße aus der Bewertung ausgenommen, da hier kein Goldstandard zum Vergleich vorlag. Für die Patienten der Kontrollgruppe, bei denen keine DSA durchgeführt worden war, wurden für alle Gefäße die Kategorie 1 („Kein relevanter Vasospasmus“, siehe Abschnitt 2.4.2) als Goldstandard angenommen.

Neben der DSA lagen bis zu zehn Ratings pro Gefäß vor (je eine CTA und vier verschiedene 4D-CTA von zwei Ratern). Falls ein Gefäß hier bei einem Verfahren nicht auswertbar war, wurde dieses Gefäß für dieses Verfahren aus der Auswertung ausgenommen. Alle anderen, bewertbaren Verfahren desselben Gefäßes wurden dennoch weiterhin berücksichtigt.

Ein diagnostischer Test sollte eine hohe Sensitivität bei einer hohen Spezifität haben und reproduzierbare Ergebnisse liefern. Daher wurden folgende Fragestellungen auf Grundlage der Daten untersucht: Welche Sensitivität und Spezifität wurden vom jeweiligen Verfahren erreicht? Wie verlässlich war dieser Test zwischen verschiedenen Ratern? Wie gut wurde der Schweregrad der Vasospasmen beurteilt? Wie einig waren sich die beiden Rater bei dieser Beurteilung?

\subsubsection{Diagnose eines Vasospasmus}

Zunächst wurde verglichen, wie gut CTA und die verschiedenen 4DCTA im Vergleich dazu geeignet sind, einen Vasospasmus zu detektieren, unabhängig von der Bewertung des Schweregrades. Hierzu wurden die Sensitivitäten von CTA und den verschieden 4D-CTA miteinander verglichen (siehe Abschnitt 2.6.1). Komplementär wurden die Spezifitäten aller Verfahren gegeneinander verglichen, um zu bewerten, wie wahrscheinlich die Verfahren eine falsch-positive Detektion von Vasospasmen suggerieren. Sowohl Sensitivität als auch Spezifität wurden für beide Rater separat bewertet.

\subsubsection{Bewertung des Vasospasmusgrades}

Weiter wurde verglichen, inwieweit CTA bzw. 4D-CTA nicht nur hinsichtlich des Vorhandenseins, sondern auch hinsichtlich des Schweregrades mit dem Goldstandard DSA übereinstimmen. Hierzu wur- 
den zwei statistische Größen betrachtet: die Pearson-Korrelation zwischen DSA und CTA bzw. 4D-CTA (siehe Abschnitt 2.6.2) sowie die Interrater-Reliabilität (siehe Abschnitt 2.6.3) zwischen DSA und CTA bzw. 4D-CTA. Dies hat den Hintergrund, dass die Interrater-Reliabilität nur perfekte Übereinstimmung mit dem Goldstandard als Übereinstimmung wertet. Der Pearson-Korrelationskoeffizient hingegen bezieht auch die Tendenz, dass Goldstandard und dagegen verglichenes Verfahren einen niedrigeren bzw. höheren Vasospasmusgrad diagnostizieren, mit ein, selbst wenn die Bewertungen nicht übereinstimmen. Sowohl Pearson-Korrelation als auch Interrater-Reliabilität wurden für beide Rater getrennt betrachtet.

\subsubsection{Interrater-Reliabilität der Vasospasmusdetektion}

Weiter wurde verglichen, inwieweit der erfahrene und der unerfahrene Rater bei ihrer Bewertung des Vorhandenseins eines Vasospasmus übereinstimmen. Hierzu wurden für jedes Verfahren die InterraterReliabilität (siehe Abschnitt 2.6.3) und Pearson-Korrelation (Abschnitt 2.6.2) zwischen den beiden Ratern bestimmt, wobei nur die Unterteilung in Normalbefund (Grad 1) und pathologischer Befund (Grad 2 oder 3) berücksichtigt wurde.

\subsubsection{Interrater-Reliabilität des Vasospasmusgrades}

Zuletzt wurde verglichen, inwieweit der erfahrene und der unerfahrene Rater nicht nur bei ihrer Bewertung des Vorhandenseins eines Vasospasmus, sondern auch in der Bewertung von dessen Schweregrad übereinstimmen. Hierzu wurde für jedes Verfahren neben der Interrater-Reliabilität (siehe Abschnitt 2.6.3) auch die Pearson-Korrelation (siehe Abschnitt 2.6.2) zwischen den beiden Ratern bestimmt. Dies hat den Hintergrund, dass die Interrater-Reliabilität nur perfekte Übereinstimmung der Rater als Übereinstimmung wertet. Analog zu Abschnitt 2.5.2 bezieht der Pearson-Korrelationskoeffizient auch die Tendenz, dass beide Rater einen niedrigeren bzw. höheren Vasospasmusgrad diagnostizieren mit ein.

\subsubsection{Aufschlüsselung nach Arterien}

Für alle oben genannten Kenngrößen wurden zur Erzielung der größtmöglichen Stichprobengröße die Bewertungen für alle Gefäße 
aller Patienten zusammengenommen herangezogen. Zusätzlich wurden alle Größen aber auch nach den einzelnen Gefäßen (ICA, MCA, ACA, PA, BA) aufgeschlüsselt ermittelt, um etwaige Schwächen von Methoden bei der Bewertung bestimmter Lokalisationen aufzudecken. Da kein Unterschied zwischen den Hemisphären zu erwarten ist, wurden hierbei linke und rechte MCA, ICA, ACA und PA zusammengefasst, um genauere statistische Aussagen zuzulassen.

\subsubsection{Bewertung von Signifikanz}

Für alle Kenngrößen Sensitivität, Spezifität, Interrater-Reliabilität und Korrelation wurde jeweils das Konfidenzintervall (KI) zum Konfidenzniveau 0,05 ermittelt. Das resultierende 95\%-Konfidenzintervall (95\%-KI) lässt somit Aussagen zu signifikanten Unterschieden zum Konfidenzniveau $5 \%$ zu: falls die ermittelten Konfidenzintervalle zweier zu vergleichender Verfahren komplett disjunkt sind, sich also nicht überlappen, so ist der Unterschied als signifikant einzustufen (Rinne 2008).

\subsection{Statistische Kenngrößen}

Im Folgenden werden die verwendeten statistischen Kenngrößen sowie die Konstruktion von deren Konfidenzintervallen erläutert.

\subsubsection{Sensitivität und Spezifität}

Für ein Diagnoseverfahren $X$ (CTA oder eines der 4D-CTA) wurden die Sensitivität und Spezifität mithilfe einer Vierfeldertafel wie folgt bestimmt (Tabelle 3). Die DSA wurde als Goldstandard herangezogen und definierte somit die "korrekte“ Unterteilung in Normalbefund (Rating 1) oder pathologischer Befund (Rating 2 oder 3). Demgegenüber steht das Rating von CTA bzw. 4D-CTA, welches bezogen auf den Goldstandard richtig negativ oder falsch positiv (bei Normalbefund) bzw. richtig positiv oder falsch negativ (bei pathologischem Befund) sein kann. In der Vierfeldertafel wurden die absoluten Häufigkeiten dieser vier Befundungen eingetragen.

Die Sensitivität wurde dann bestimmt aus der Anzahl aller positiven Tests $a$ aus der marginalisierten Häufigkeit aller vorhandenen Vasospasmen $a+c$ : 


$$
\text { Sen }=\frac{a}{a+c} \text {. }
$$

Dementsprechend ergibt sich die Spezifität aus der Anzahl aller negativen Testergebnisse und der marginalisierten Häufigkeit aller Gefäße mit Normalbefund in der DSA:

$$
\text { Spe }=\frac{b}{b+d} .
$$

Das Konfidenzintervall für Sensitivität und Spezifität wurde gemäß der Methode von Clopper und Pearson bestimmt (Rinne 2008). Dieses Verfahren wurde gewählt, da es speziell bei kleiner Stichprobengröße eine exaktere und insbesondere konservativere Abschätzung des Konfidenzintervalls im Vergleich zur weiter verbreiteten Näherung durch eine Normalverteilung bietet (Rinne 2008). Die Auswertung wurde realisiert mit der Toolbox Statsmodels: Statistics in Python (Perktold et al. 2018).

\section{Tabelle 3: Vierfeldertafel.}

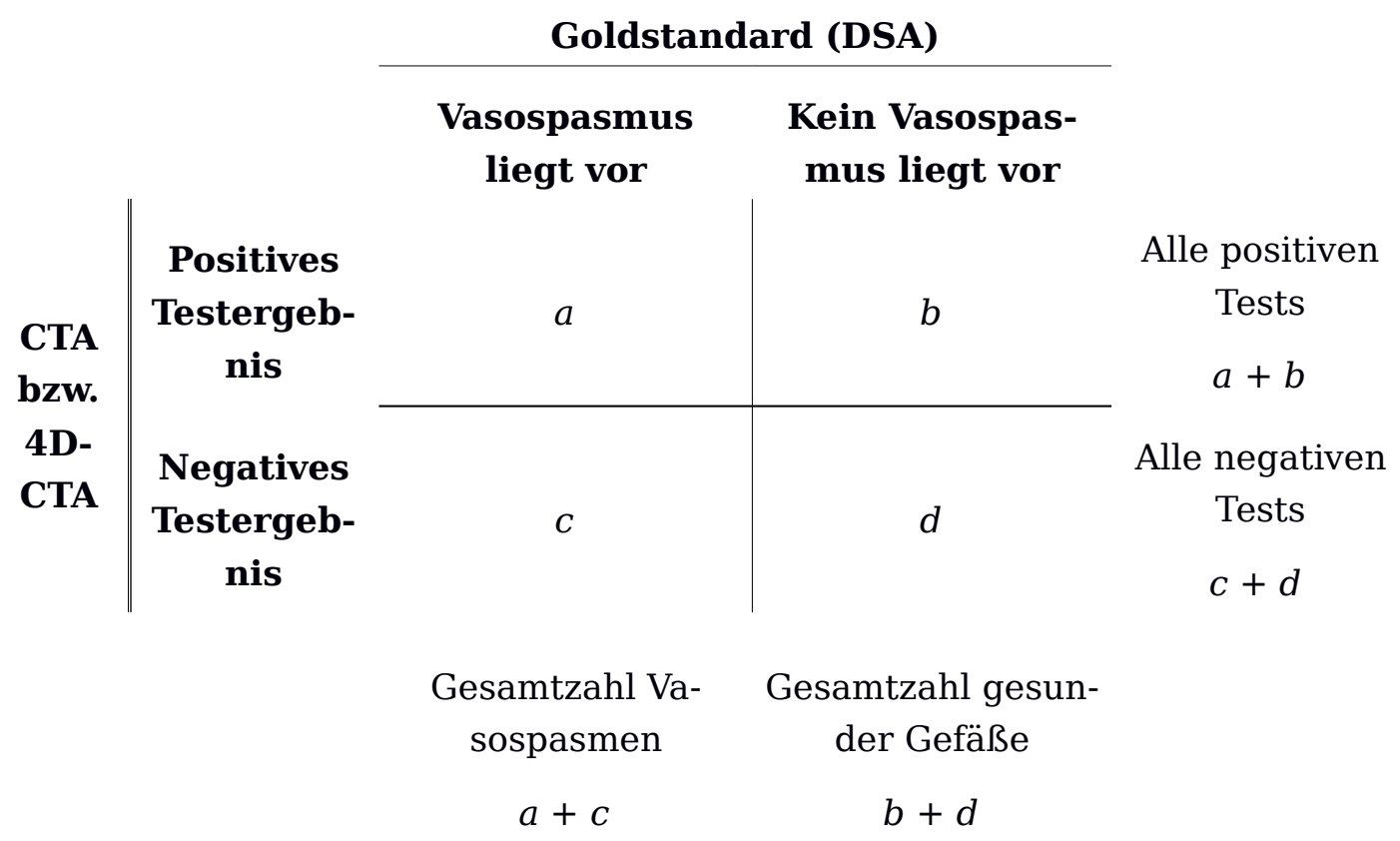

In der Vierfeldertafel sind die absoluten Häufigkeiten aufgeführt, mit denen Vasospasmen diagnostiziert oder nicht diagnostiziert wurden, in Abhängigkeit vom Vorhandensein eines Vasospasmus (definiert durch Goldstandard DSA). 


\subsubsection{Pearson-Korrelation}

Die Pearson-Korrelation zwischen zwei Größen X und Y wurde mithilfe des Pearson-Korrelationskoeffizienten $r$ bestimmt. Der PearsonKorrelationskoeffizienten gibt Aufschluss über den linearen Zusammenhang zwischen den Größen X und Y. Er charakterisiert also deren Tendenz, in die gleiche Richtung abzuweichen. Der Korrelationskoeffizient nimmt Werte zwischen -1 und 1 an. Dabei bedeutet ein Korrelationskoeffizient von $r=1$ einen perfekten linearen Zusammenhang zwischen den Größen, ein Korrelationskoeffizient von $r=-1$ ein perfekt entgegengesetzten linearen Zusammenhang. Ein Korrelationskoeffizient von $r=0$ zeigt an, dass kein linearer Zusammenhang festzustellen ist (Rinne 2008).

Konfidenzintervalle wurden nach Rinne (2008) bestimmt. Hierzu wurde der Fisher-Z-Score berechnet. Dieser Fisher-Z-Score ist approximativ normalverteilt mit Standardfehler

$$
\mathrm{SE}=\frac{1,03}{\sqrt{n-3}}
$$

Hierbei wurde die konservative Korrektur des Standardfehlers für kleine Stichprobengrößen nach Fieller et al. (1957) gewählt. Mithilfe dieses Standardfehlers sowie den 2,5\% bzw. 97,5\%-Perzentilen der Standard-Normalverteilung wurde ein 95\%-KI für die Z-Scores geschätzt. Hieraus wurde mithilfe der inversen Fisher-Transformation das 95\%-KI für den Korrelationskoeffizienten bestimmt.

\subsubsection{Interrater-Reliabilität}

Die Übereinstimmung der beiden Rater, die Interrater-Reliabilität, wurde mithilfe von Cohen's $к$ quantifiziert. Dieses Maß vergleicht den Anteil der Beurteilungen, bei denen beide Rater übereinstimmen, mit dem Anteil der aus bloßem Zufall erwartbaren Übereinstimmungen. Die Interrater-Reliabilität ist also gleich 1, wenn die Beurteilungen beider Rater perfekt übereinstimmen, und gleich 0 , wenn die Übereinstimmungen nur auf Zufallsniveau liegen. Dazwischen hat sich nach Landis und Koch (1977) folgende Bewertung bewährt: Werte zwischen 0,2 und 0,4 indizieren ausreichende Übereinstimmung, Werte bis 0,6 mittelmäßige Übereinstimmung, Werte bis 0,8 
beachtliche Übereinstimmung und Werte zwischen 0,8 und 1,0 fast vollkommene Übereinstimmung.

In dieser Arbeit wurde die Interrater-Reliabilität mithilfe der Toolbox Statsmodels: Statistics in Python (Seabold und Perktold 2010) bestimmt, die auch 95\%-Konfidenzintervalle bestimmt. 


\section{Ergebnisse}

In diesem Kapitel werden die Ergebnisse der im Rahmen dieser Promotion durchgeführten Studie dargestellt. Zunächst werden grundsätzliche Beobachtungen zum Patientenkollektiv vorgestellt. Dies umfasst epidemiologische Daten (Abschnitt 3.1), die Lokalisationen der Aneurysmen (Abschnitt 3.2), die Auswertbarkeit der Gefäße in der DSA hinsichtlich Vasospasmen (Abschnitt 3.3), die Lokalisationen der auswertbaren Vasospasmen (Abschnitt 3.4), den Zeitpunkt des Auftretens der Vasospasmen nach SAB (Abschnitt 3.5) sowie die eingesetzten Behandlungsmethoden der SAB mit Bewertung hinsichtlich des Auftretens von Vasospasmen im Verlauf (Abschnitt 3.6). Daraufhin werden die Ergebnisse mit eigentlichem Bezug zu den untersuchten Bildgebungsverfahren vorgestellt. Dies sind zum einen die potentiell erreichbare Dosisreduktion (Abschnitt 3.7) und ein subjektiver, exemplarischer Vergleich der Auswertung von CTA und 4D-CTA (Abschnitt 3.8). Zum anderen sind dies die Vergleiche zwischen CTA und 4D-CTA hinsichtlich der Diagnose eines Vasospasmus (Sensitivität und Spezifität, Abschnitt 3.9), der Bewertung des Vasospasmusgrades (Abschnitt 3.10) sowie der Interrater-Reliabilität dieser beiden Größen (Abschnitte 3.11 und 3.12).

\subsection{Epidemiologie}

Von den ausgewählten 115 Patienten mit SAB waren 44 männlich (39\%) und 71 weiblich (61\%). Die Altersverteilung ist in Abbildung 5 a gezeigt. Bei einem minimalen Alter von 25 Jahren und einem maximalen Alter von 89 Jahren betrug das mediane Alter des Patientenkollektivs 54 Jahre, das mittlere Alter 55 Jahre.

Die 31 Patienten (13 Männer und 18 Frauen) mit einem interventionell behandlungsbedürftigen Vasospasmus hatten ein mittleres Alter von 51 Jahren (Median: 53 Jahre) bei einem minimalen Alter von 30 Jahren und einem maximalen Alter von 72 Jahren (Abbildung 5b). 


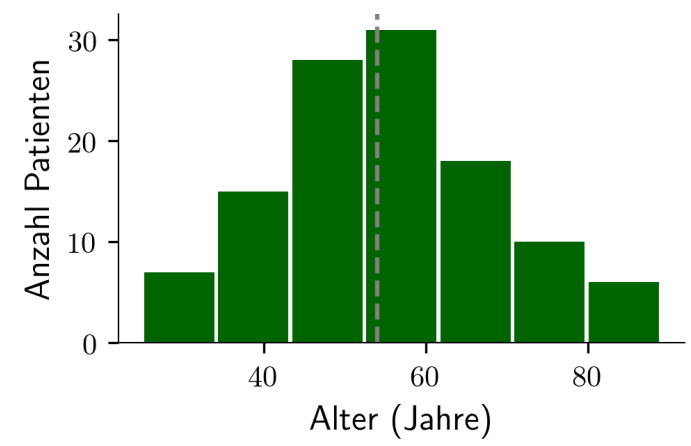

b

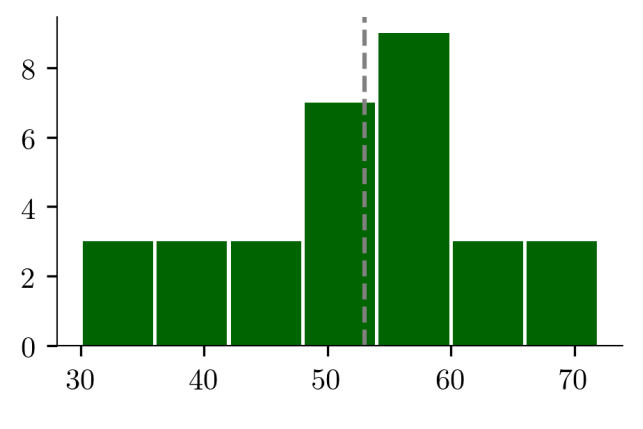

Abbildung 5: Epidemiologie der SAB und Vasospasmen. a. Altersverteilung der 115 ausgewählten Patienten mit SAB. b. Altersverteilung der 31 Patienten mit behandlungsbedürftigen Vasospasmus nach SAB. Die graue Linie zeigt jeweils den Median an.

\subsection{Lokalisation der Aneurysmen}

Im Folgenden werden die Lokalisationen der Aneurysmen dargestellt: zum einen für alle Aneurysmen, die Ursache einer SAB waren, und zum anderen für die Aneurysmen, die Ursache einer SAB waren, in deren Verlauf ein Vasospasmus auftrat.

\subsubsection{Lokalisation der für SAB ursächlichen Aneurysmen}

Die Auswertung der 108 Patienten mit SAB nach rupturiertem Aneurysma (Kollektiv (iii) in Abbildung 1) ergab bezüglich der Lokalisation des ursächlichen Aneurysmas folgende Verteilung (Abbildung 6a): Am häufigsten konnten Aneurysmen der Arteria communicans anterior (ACOM) nachgewiesen werden (40 Fälle), gefolgt von Aneurysmen der MCA (18 Fälle) und der ICA (15 Fälle). Aneurysmen der ACA traten deutlich seltener auf (fünf Fälle). Aneurysmen des hinteren Kreislaufs waren seltener ursächlich für die SAB (A. Posterior communicans (PCOM): 8 Fälle, PCA: 4 Fälle, A. Superior cerebelli (SCA): 3 Fälle, BA: 14 Fälle, A. Inferior anterior cerebelli (AICA): 0 Fälle, A. Inferior posterior cerebelli (PICA): 4 Fälle, A. Vertebralis (VA): 2 Fälle). Hierbei lagen bei fünf Patienten zwei Aneurysmen vor. 

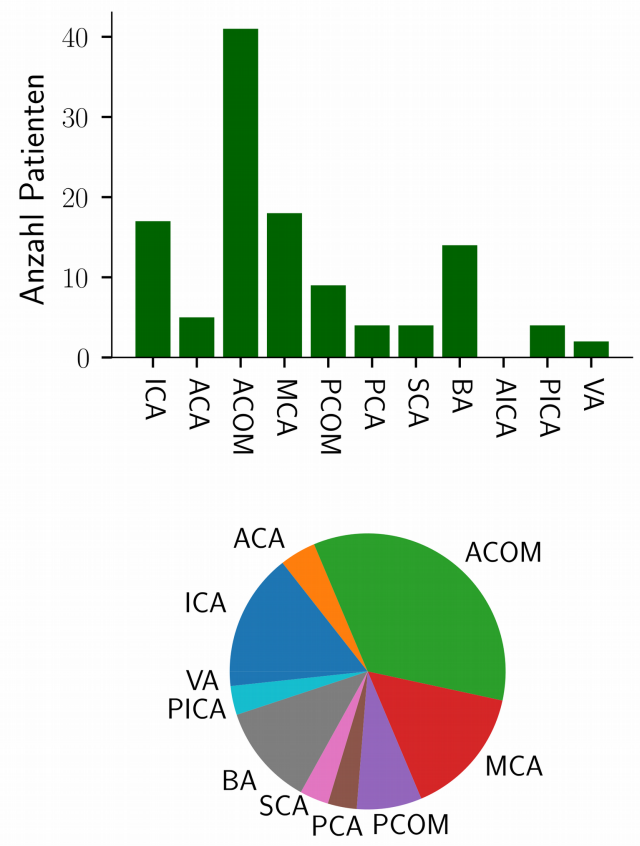

b
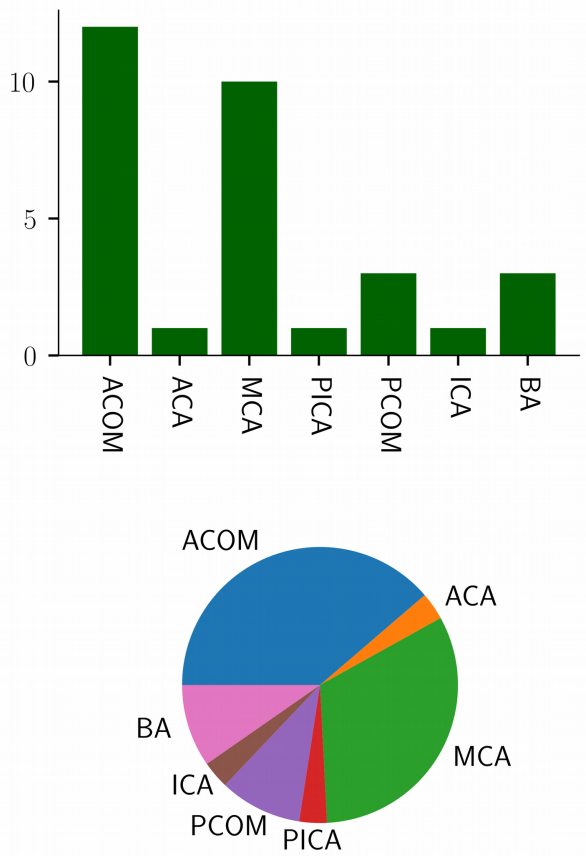

Abbildung 6: Lokalisation der Aneurysmen. Jeweils gezeigt sind oben das Histogramm mit Anzahl der Aneurysmen je unterschiedenem Gefäß sowie unten das Kreisdiagramm mit deren Anteilen an allen Aneurysmen. a. Daten für alle 85 Patienten mit Aneurysma, das für eine SAB verantwortlich war. b. Daten für die 31 Patienten mit späterem Vasospasmus.

\subsubsection{Lokalisation der für SAB ursächlichen Aneurysmen mit späterem Vasospasmus}

Für die 31 Patienten mit Vasospasmus nach SAB (Kollektiv (iv) in Abbildung 1) zeigte sich, dass ACOM- und MCA-Aneurysmen einen vergleichbaren Anteil an allen Aneurysmen hatten (Abbildung 6b): in zwölf Fällen ein lag ein ACOM- und in zehn Fällen ein MCA-Aneurysma vor. Dagegen gab es ICA- und ACA-Aneurysmen in dieser Gruppe nur bei jeweils einem Patienten. In je drei Fällen lag ein PCOM-oder BA- Aneurysma vor, in einem Fall ein PICA-Aneurysma. 


\subsection{Auswertbarkeit der DSA hinsichtlich Vasospasmus}

Die Auswertbarkeit der Gefäße hing stark von der untersuchten Arterie ab. Insgesamt wurden 166 Gefäße untersucht. Die DSA von 58 Gefäßen war dabei nicht auswertbar, sodass diese komplett aus der Analyse entfernt wurden. Aufgeschlüsselt pro Arterie ergab sich ein differenzierteres Bild (Abbildung 7a,b). Es konnten ca. 90\% aller Gefäße für ACA, ICA und MCA bewertet werden. Demgegenüber stehen jedoch BA und PA, für die nur $20 \%$ oder weniger der Gefäße beurteilt werden konnten.

a

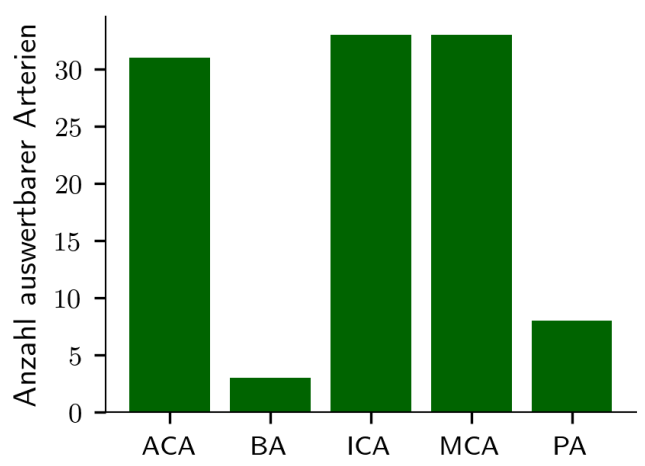

C

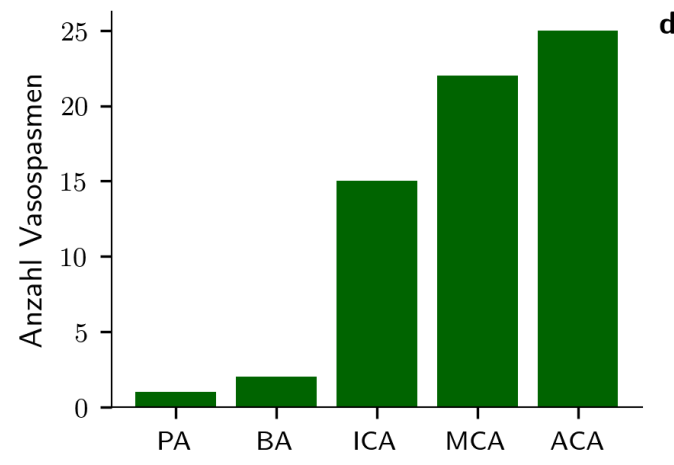

b

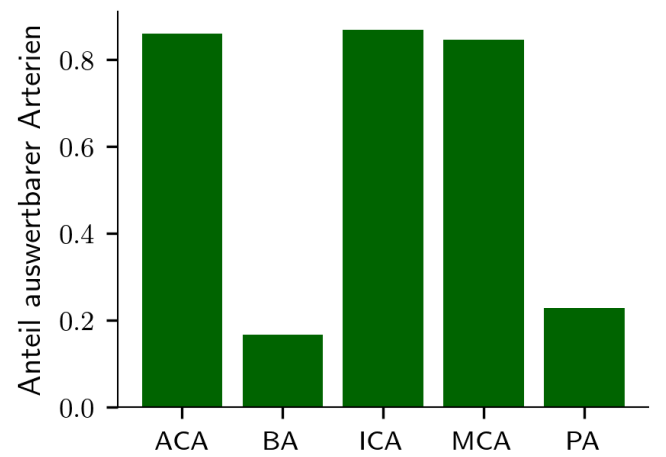

d

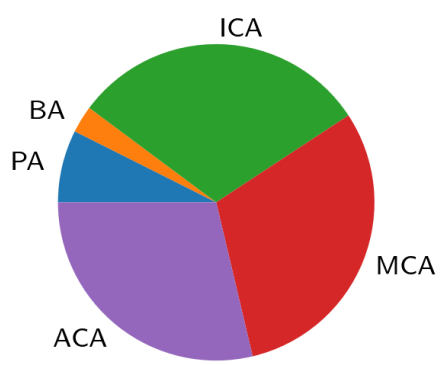

Abbildung 7: Auswertung der Vasospasmen (DSA). a. Anzahl aller in der DSA beurteilbaren Patienten, aufgeschlüsselt pro Arterie (Hemisphären nicht weiter aufgeschlüsselt). b. Relativer Anteil aller in der DSA beurteilbaren Patienten im Verhältnis zu allen Patienten, aufgeschlüsselt pro Arterie (Hemisphären nicht weiter aufgeschlüsselt). c. Anzahl der Vasospasmen pro Arterie, identifiziert mit Goldstandard DSA. d. Relativer Anteil der Gefäße an allen Vasospasmen. 


\subsection{Lokalisation der Vasospasmen}

Am häufigsten wurden Vasospasmen in der ACA (25 Vasospasmen) festgestellt, gefolgt von MCA (22) und ICA (15). Vasospasmen in der BA (2) und der PA (1) wurden deutlich seltener festgestellt. Korrigiert man diese Werte um die Auswertbarkeit der Gefäße, so ergaben sich korrigierte erwartete Häufigkeiten von 28 (25/89\%) Vasospasmen in der ACA, 24 (22/89\%) in der MCA, 18 (15/84\%) in der ICA, 13 (2 / 15\%) in der BA sowie 5 (1 / 20\%) in der PA. Die Differenz zwischen den Häufigkeiten von BA und PA bzw. ACA, MCA und ICA war also kleiner, als wenn die unterschiedliche Auswertbarkeit nicht mit einbezogen wird. Dennoch waren Vasospasmen in BA und PA nach wie vor seltener als in ACA, MCA und ICA.

\subsection{Zeitliches Auftreten der Vasospasmen}

Das größte Risiko für das Entwickeln von Vasospasmen bestand zwischen dem dritten und siebten Tag nach SAB. Die überwiegende Zahl der Vasospasmen (18 von 31, Abbildung 8) wurden bei der zweiten Kontrolluntersuchung diagnostiziert, traten also zwischen der ersten Kontrolluntersuchung an Tag drei und der zweiten Kontrolluntersu-

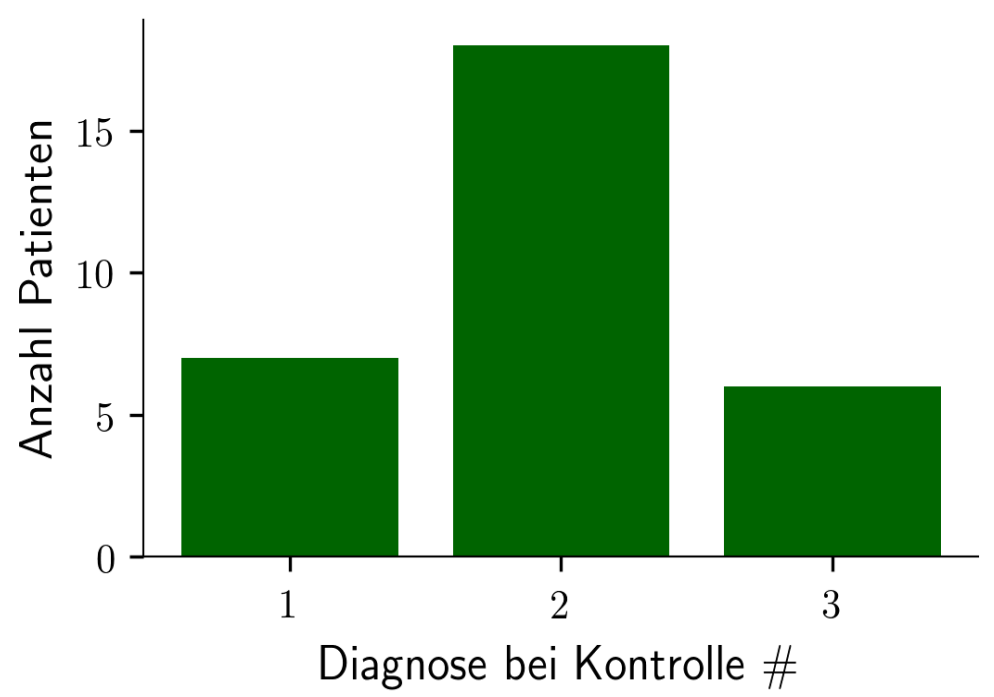

Abbildung 8: Zeitliches Auftreten von Vasospasmen. Gezeigt ist, bei welcher Kontrolluntersuchung (Tag drei, sieben und zehn) der Vasospasmus diagnostiziert wurde. 
chung an Tag sieben auf. Innerhalb der ersten drei Tage nach SAB sowie zwischen Tag sieben und zehn traten sieben bzw. sechs Vasospasmen auf.

\subsection{Behandlung der SAB und vasospasmusbedingten Infarkte}

Von den 108 Patienten mit bestätigter SAB nach rupturiertem Aneurysma wurden bei zwei Patienten zwei Aneurysmen identifiziert, die im Folgenden unabhängig gezählt wurden. Bei zwei Patienten war die initiale Behandlung frustran, sodass im Verlauf eine zweite Behandlung nötig wurde, die beide separat gezählt wurden. Zur Behandlung der SAB erhielten 51 Patienten ein Clipping des Aneurysmas (45,1\%), 60 Patienten eine Coil-Embolisation. Die Coil-Embolisationen ließen sich noch einmal aufschlüsseln in 45 Patienten mit CoilEmbolisation (39,8\%), 6 Patienten mit Coil-Embolisation und Ballon (5,3\%) und 9 Patienten mit Coil-Embolisation und Stent (7,9\%). Ein Aneurysma blieb unversorgt (0,9\%).

31 Patienten des Patientenkollektivs entwickelten einen interventionell behandlungsbedürftigen Vasospasmus $(28,7 \%)$. Von allen Patienten mit interventionell behandlungsbedürftigem Vasospasmus zeigte sich insgesamt bei 14 Patienten (45\%) ein vasospasmusbedingter Infarkt im Verlauf. 17 Patienten (55\%) entwickelten keinen Infarkt, der mit dem Vasospasmus assoziiert war.

20 Patienten mit einem interventionell behandlungsbedürftigen Vasospasmus hatten vorab ein Clipping des rupturierten Aneurysmas erhalten. Acht dieser 20 Patienten, die geclippt worden waren, erlitten einen Infarkt im Verlauf (40\%). 13 Patienten mit einem interventionell behandlungsbedürftigen Vasospasmus hatten eine Coil-Embolisation des rupturierten Aneurysmas erhalten. Sieben dieser Patienten (54\%) entwickelten im Verlauf einen Infarkt im Vasospasmusgebiet. Zwei Patienten erhielten sowohl eine Coil-Embolisation als auch ein Clipping, weil die initiale Behandlung nicht erfolgreich war. Diese Behandlungen wurden hier einzeln gezählt. Einer dieser Patienten erlitt im Verlauf einen Infarkt. 


\subsection{Potenzielle Dosisreduktion}

Die Dosisreduktion wurde für zwei Indizes zur Abschätzung der Strahlendosis separat untersucht: Computed Tomography Dose Index (CTDI) und Dosislängenprodukt (DLP). Von 31 Patienten lagen für zwei Patienten keine Informationen zur Dosisbelastung während der Untersuchungen vor. Die anderen 29 Patienten waren über alle Kontrolluntersuchungen insgesamt einem mittleren CTDI von 828,3 mGy (Median: 828,9 mGy), bzw. einem mittleren DLP von 10470,7 mGy cm (Median: 10443,0 mGy cm) ausgesetzt.

Die Verteilung der möglichen Dosisreduktionen über das gesamte Untersuchungsprotokoll bei Verzicht auf die drei CTA-Untersuchun-
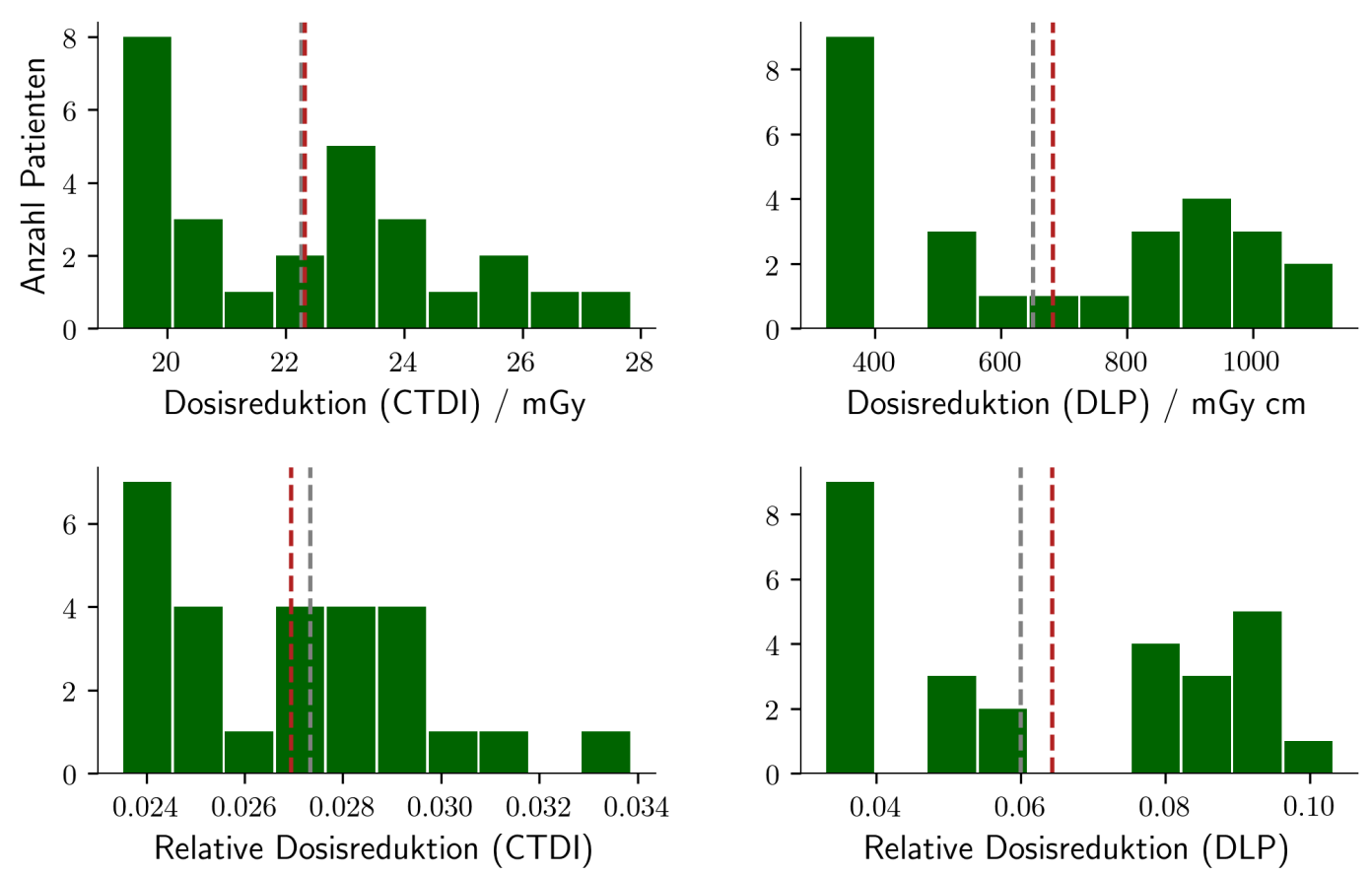

Abbildung 9: Potenzielle Dosisreduktionen. Jeweils gezeigt ist oben die absolute Dosisreduktion bei Verzicht auf die CTA-Untersuchungen über die gesamte Routineuntersuchung, unten die relative Dosisreduktion für a. den Computed Tomography Dose Index (CTDI) als Dosisabschätzung und b. das Dosislängenprodukt (DLP) als Dosisabschätzung. Die graue Linie stellt jeweils den Median, die rote den Mittelwert dar. 
gen in Abbildung 9 gezeigt. Für das CTDI ließe sich bei Verzicht auf die CTA-Untersuchungen eine mittlere Dosisreduktion von 22,3 mGy erzielen (Median 22,3 mGy), was einer mittleren Reduktion um 2,6\% entspricht (Median 2,7\%). Für das DLP ließe sich bei Verzicht auf die CTA-Untersuchungen eine mittlere Dosisreduktion von 683,1 mGy cm erzielen (Median 651,0 mGy cm), was einer mittleren Reduktion um 6,4\% entspricht (Median 6,0\%).

\subsection{Subjektiver Eindruck der Bilddiagnostik}

Die Bewertung der Gefäße mittels Standard-CTA und 4D-CTA wurde zunächst qualitativ verglichen. Exemplarische Bildgebungen von Standard-MIP und MIP aus früharterieller Darstellung aus 4D-CTA sind in Abbildung 10 dargestellt. Der 70-jährige Patient war mit SAB bei rupturiertem Aneurysma der Arteria basilaris aufgenommen und mit Clipping behandelt worden. Im Verlauf entwickelte der Patient mäßig ausgeprägte Vasospasmen im Bereich der linken ICA, MCA
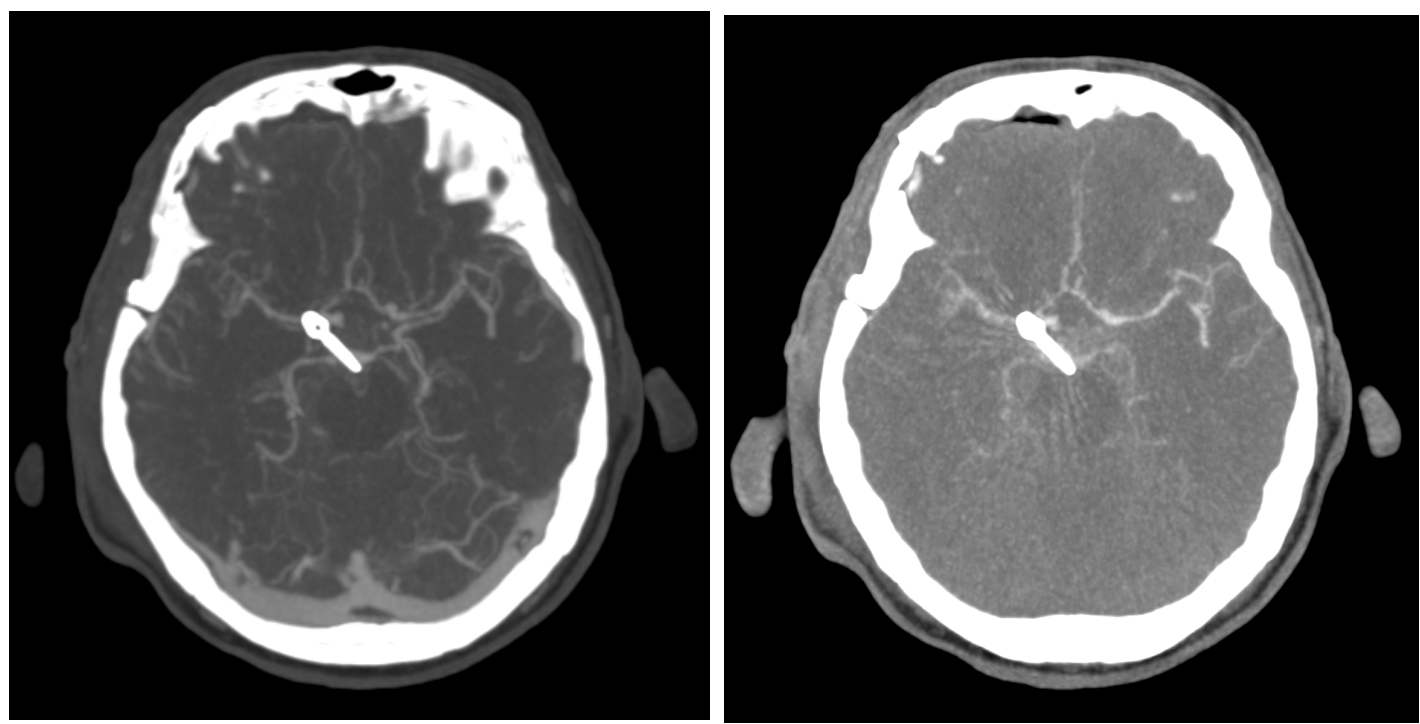

Abbildung 10: Vergleich von Standard-MIP und früharterieller 4D-CTA. Exemplarische Darstellungen für einen 70-jährigen Patienten mit Vasospasmen im Bereich der linken ICA, MCA und ACA sowie der rechten MCA und ACA nach SAB bei rupturiertem und geclipptem Basilaris-Aneurysma. Links: Standard-MIP aus CTA. Rechts: MIP mit $10 \mathrm{~mm}$ Schichtdicke aus früharterieller Darstellung der 4DCTA. 
und ACA sowie der rechten MCA und ACA. Der subjektive Eindruck beider Rater befand die früharterielle 4D-CTA als eindeutiger zu beurteilen als die Standard-MIP. Aus Abbildung 10 lässt sich dies einfach erklären. Die Standard-MIP wurde zu einem Zeitpunkt aufgenommen, an dem Arterien und Venen gleichermaßen dargestellt werden. Daher kam es in der MIP zu einer Überlagerung von Arterien und Venen, die die Beurteilung des Gefäßstatus der Arterien erschwert. Im Gegensatz dazu erlaubte die früharterielle Darstellung eine Darstellung fast ausschließlich der Arterien ohne venöse Überlagerung. Dies erleichterte die Bewertung des arteriellen Gefäßstatus.

\subsection{Diagnose eines Vasospasmus}

Zunächst werden die Ergebnisse für die Diagnose eines Vasospasmus, unabhängig von dessen Schweregrad, präsentiert. Dafür wurden gemäß Abschnitt 2.6.1 die Sensitivitäten und Spezifitäten von CTA und 4D-CTA miteinander verglichen.

Alle Ergebnisse sind hier zunächst zusammengefasst dargestellt und in den darauf folgenden Abschnitten im Detail aufgeschlüsselt. Zusammengefasst lässt sich sagen, dass die 4D-CTA der konventionellen CTA mindestens ebenbürtig bei der Detektion von Vasospasmen war, da keines der 4D-CTA-Verfahren eine signifikant schlechtere Sensitivität oder Spezifität gegenüber der CTA aufwies.

Im Vergleich der verschiedenen 4D-CTA-Verfahren war konsistent über beide Rater, über Spezifität und Sensitivität sowie über alle einzelnen Gefäße die früharterielle Rekonstruktion der spätarteriellen Rekonstruktion überlegen. Die folgenden Schlussfolgerungen beziehen sich daher auf die früharterielle Rekonstruktion. Weiterhin ergab die MIP-Schichtdicke von $10 \mathrm{~mm}$ eine höhere Sensitivität als die MIPSchichtdicke von $5 \mathrm{~mm}$, bei der Spezifität war die Lage umgekehrt. Die Unterschiede zwischen den Schichtdicken waren in beiden Fällen jedoch gering und nicht signifikant.

Während der erfahrene Rater eine etwas höhere Sensitivität mit der 4D-CTA gegenüber der konventionellen CTA erreichen konnte, profitierte insbesondere der unerfahrene Rater von der 4D-CTA (Abschnitt 3.9.1). Hier ergab sich ein signifikanter Anstieg der Sensitivi- 
tät von $52 \%$ auf $80 \%$. Dem gegenüber steht eine leichte, aber nicht signifikante Abnahme der Spezifität beim unerfahrenen Rater, während die Spezifität des erfahrenen Raters für CTA und 4D-CTA gleich war (Abschnitt 3.9.2).

Für die einzelnen Gefäße ergab sich ein hiermit konsistentes Bild. Man muss allerdings feststellen, dass die Vasospasmen der BA und PA mit keinem Verfahren adäquat beurteilbar waren. Vasospasmen des vorderen Kreislaufs waren hingegen zuverlässig bewertbar, wobei der unerfahrene Rater hier besonders von der 4D-CTA profitierte.

\subsubsection{Sensitivität}

Zusammengefasst lässt sich weder für den unerfahrenen Rater A, noch für den erfahrenen Rater B eine signifikante Verschlechterung irgendeiner 4D-CTA gegenüber der konventionellen CTA feststellen.

ACA Rater A (unerfahren)

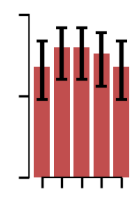

MCA

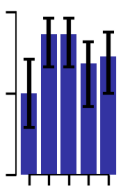

ICA

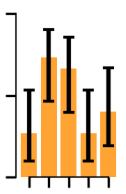

PA

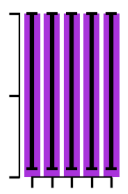

BA

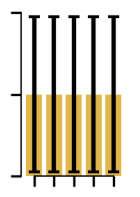

Rater B (erfahren)
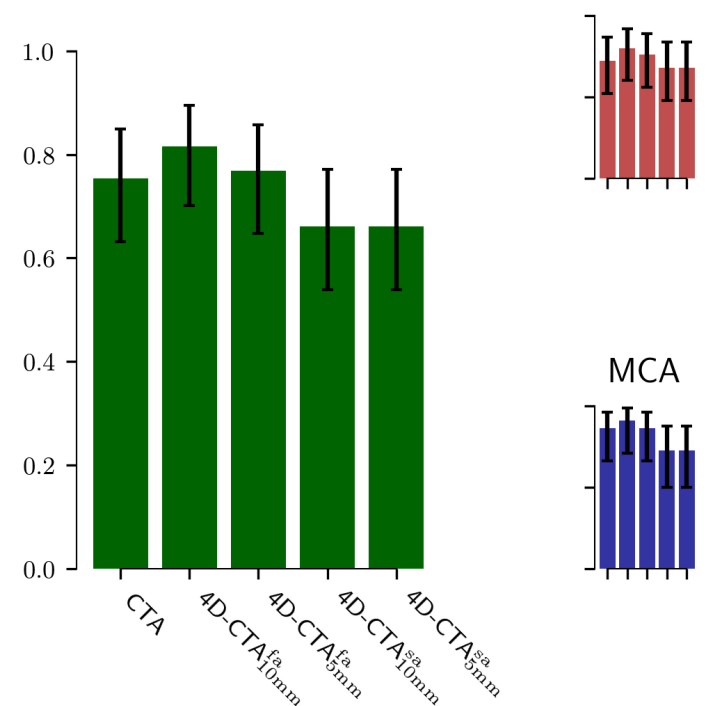

BA

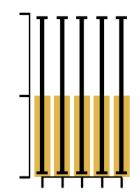

PA

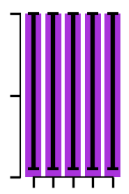

ACA

MCA

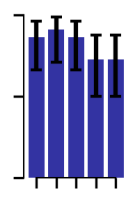

ICA

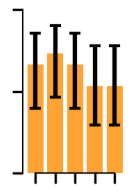

Abbildung 11: Sensitivität der Vasospasmusdetektion. Sensitivität von Rater A (links) und Rater B (rechts) über alle Gefäße (grün) und über die einzelnen betrachteten Gefäße einzeln aufgeschlüsselt. Rote Balken zeigen signifikante Unterschiede (5\%-Konfidenz). 
Die früharterielle 4D-CTA mit 5 mm MIP-Schichtdicke lieferte konsistent die beste Sensitivität aller Verfahren (Abbildung 11).

Für den unerfahrenen Rater A ergab sich für die konventionelle CTA eine Sensitivität von 52\% (95\%-KI [39\%, 65\%]) über alle Gefäße. Demgegenüber steht eine Sensitivität von 80\% (95\%-KI [69\%, 88\%]) für die $4 \mathrm{D}-\mathrm{CTA}_{\mathrm{fa}, 5 \mathrm{~mm}}$, von $78 \%(95 \%-\mathrm{KI}[67 \%, 87 \%])$ für die $4 \mathrm{D}$ $\mathrm{CTA}_{\mathrm{fa}, 10 \mathrm{~mm}}$, von $62 \%(95 \%-\mathrm{KI}[49 \%, 73 \%])$ für die $4 \mathrm{D}-\mathrm{CTA}_{\mathrm{sa}, 10 \mathrm{~mm}}$, sowie $63 \%(95 \%-K I[50 \%, 74 \%])$ für die $4 \mathrm{D}-\mathrm{CTA}_{\mathrm{sa}, 5 \mathrm{~mm}}$. Daher waren für Rater A alle 4D-CTA der konventionellen CTA überlegen, wobei dieser Unterschied nur für die früharterielle 4D-CTA signifikant (5\%-Konfidenzniveau) war. Ohne alle exakten Werte zu geben, zeigt sich ein ähnliches Bild für alle einzelnen Arterien. ACA, MCA und ICA zeigen den gleichen Trend wie zuvor über alle Gefäße berichtet. Hierbei waren allerdings aufgrund der kleineren Stichprobengröße keine der Unterschiede statistisch signifikant. Für BA und PA ergaben sich exakt die gleichen Sensitivitäten für alle betrachteten Verfahren.

Die Ergebnisse für den erfahrenen Rater B waren hiermit konsistent. Für die konventionelle CTA ergab sich eine Sensitivität von 75\% (95\%-KI [63\%, 85\%]) über alle Gefäße. Die Sensitivität des erfahrenen Raters war also höher als die des unerfahrenen Raters. Demgegenüber steht eine Sensitivität von 82\% (95\%-KI [70\%, 89\%]) für die $4 \mathrm{D}-\mathrm{CTA}_{\mathrm{fa}, 5 \mathrm{~mm}}$, von $77 \%$ (95\%-KI $\left.[67 \%, 86 \%]\right)$ für die $4 \mathrm{D}-\mathrm{CTA}_{\mathrm{fa}, 10 \mathrm{~mm}}$, von $66 \%(95 \%-K I[54 \%, 77 \%])$ für die $4 \mathrm{D}-\mathrm{CTA}_{\mathrm{sa}, 10 \mathrm{~mm}}$ sowie $66 \%(95 \%-\mathrm{KI}$ $[54 \%, 77 \%])$ für die $4 \mathrm{D}-\mathrm{CTA}_{\mathrm{sa}, 5 \mathrm{~mm}}$. Diese Sensitivitäten waren kaum höher als die des unerfahrenen Raters. Bezogen auf Rater B sind aber wieder die früharteriellen 4D-CTA der konventionellen CTA überlegen, allerdings aufgrund der höheren Sensitivität der CTA nicht signifikant. Die spätarterielle 4D-CTA lieferte etwas geringere Sensitivitäten als die konventionelle CTA, allerdings wiederum nicht signifikant. Auch für Rater B zeigte sich ein ähnliches Bild für jede der einzelnen Arterien. ACA, MCA und ICA zeigten auch hier den gleichen Trend wie zuvor über alle Gefäße berichtet, ohne dass sich einer der Unterschiede signifikant wäre. Für BA und PA ergaben sich exakt die gleichen Sensitivitäten für alle betrachteten Verfahren. 

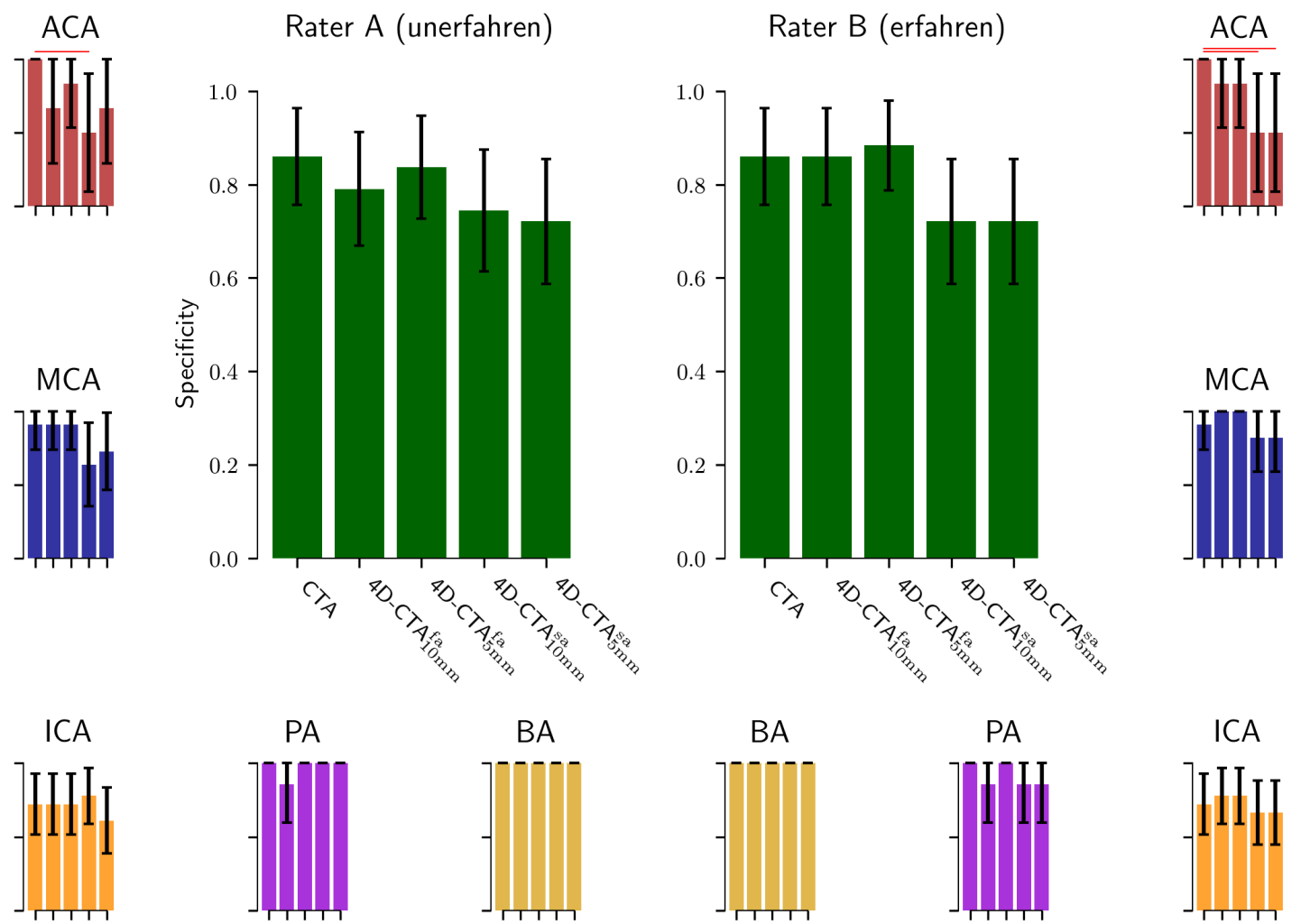

Abbildung 12: Spezifität der Vasospasmusdetektion. Spezifität von Rater A (links) und Rater B (rechts) über alle Gefäße (grün) und über die einzelnen betrachteten Gefäße einzeln aufgeschlüsselt. Rote Balken zeigen signifikante Unterschiede (5\%-Konfidenz).

\subsubsection{Spezifität}

Zusammengefasst ließ sich weder für den unerfahrenen Rater A, noch für den erfahrenen Rater B eine signifikante Verschlechterung der Spezifität irgendeiner 4D-CTA gegenüber der konventionellen CTA feststellen (Abbildung 12).

Für den unerfahrenen Rater A ergab sich für die konventionelle CTA eine Spezifität von 86\% (95\%-KI [76\%, 96\%]) über alle Gefäße. Demgegenüber stand eine Spezifität von 79\% (95\%-KI [67\%, 91\%]) für die $4 \mathrm{D}-\mathrm{CTA}_{\mathrm{fa}, 5 \mathrm{~mm}}$, von $84 \%(95 \%-\mathrm{KI}[73 \%, 95 \%])$ für die $4 \mathrm{D}-\mathrm{CTA} \mathrm{fa}_{\mathrm{fa}, 10 \mathrm{~mm}}$, von $74 \%(95 \%-K I[61 \%, 87 \%])$ für die $4 \mathrm{D}-\mathrm{CTA}_{\mathrm{sa}, 10 \mathrm{~mm}}$, sowie $72 \%(95 \%$ -

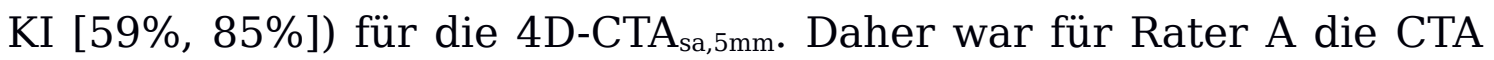
zwar spezifischer als alle 4D-CTA-Verfahren, allerdings war keiner dieser Unterschiede signifikant. Für die früharterielle 4D-CTA war 
die Differenz zudem kleiner als für die spätarterielle 4D-CTA. Speziell die früharterielle 4D-CTA mit einer Schichtdicke von $10 \mathrm{~mm}$ erreichte quasi die gleiche Spezifität wie die konventionelle CTA (2\% Differenz).

Für den erfahrenen Rater B zeigte sich ein im Detail anderes, von der Gesamtbewertung aber ähnliches Bild. Für die konventionelle CTA ergab sich eine Spezifität von 86\% (95\%-KI [76\%, 96\%]) über alle Gefäße. Die gleiche Spezifität von 86\% (95\%-KI [76\%, 96\%]) zeigte sich für die $4 \mathrm{D}-\mathrm{CTA}_{\mathrm{fa}, 5 \mathrm{~mm}}$. Demgegenüber stand eine marginal höhere Spezifität von $88 \%(95 \%-K I$ [79\%, 98\%]) für die 4D-CTA fa, $10 \mathrm{~mm}$. Die 4D-CTA ${ }_{\mathrm{sa}, 10 \mathrm{~mm}}$ und die $4 \mathrm{D}-\mathrm{CTA}_{\mathrm{sa}, 5 \mathrm{~mm}}$ erreichten die gleiche Spezifität von $72 \%(95 \%$-KI [59\%, 85\%]). Auch für Rater B war keiner dieser Unterschiede signifikant.

Für die Bewertungen der einzelnen Arterien zeigte sich ein inhomogenes Bild. Keiner der Rater bewertete mit der konventionellen CTA falsch-positive Vasospasmen der ACA (100\% Spezifität, 95\%-KI [100\%, 100\%]). Keines der 4D-CTA-Verfahren konnte dieselbe Spezifität erreichen, speziell für die ACA waren einige 4D-CTA-Verfahren auch signifikant schlechter hinsichtlich der Spezifität. Für die anderen Gefäße (MCA, ICA, PA, BA) gab es keine signifikanten Unterschiede zwischen den Verfahren. Insgesamt zeigte sich auch hier, dass fast ausnahmslos die früharterielle 4D-CTA eine höhere Spezifität erzielte als die spätarterielle 4D-CTA.

\subsection{Bewertung des Vasospasmusgrades}

Abgesehen von der bloßen Diagnose eines Vasospasmus wurde auch die Bewertung von dessen Schweregrad untersucht. Hierzu kamen zwei Methoden zum Einsatz: die Interrater-Reliabilität zwischen der DSA als Goldstandard und der dagegen getesteten Methode sowie die lineare Korrelation zwischen beiden Bewertungen.

Beide Herangehensweisen zeigten ein konsistentes Bild. Alle 4DCTA-Verfahren erreichten sowohl für den erfahrenen als auch den unerfahrenen Rater zumindest eine etwas höhere Übereinstimmung mit der DSA-Bewertung im Vergleich zur konventionellen CTA. Dieser Unterschied war für die früharterielle 4D-CTA ausgeprägter als 
für die spätarterielle 4D-CTA. Im Folgenden werden diese Resultate im Detail besprochen.

\subsubsection{Cohen's $k$}

Die Interrater-Reliabilität zwischen CTA bzw. 4D-CTA und der DSA als Goldstandard stellt eine mögliche Quantifizierung der Übereinstimmung der jeweiligen Methoden dar. Die Ergebnisse sind in Abbildung 13 dargestellt. Über alle Gefäße erreichte der unerfahrene Rater A mit der CTA eine Übereinstimmung von 0,20 (95\%-KI [0,12, $0,28]$ ). Damit wurde hiermit nur „etwas Übereinstimmung“ erzielt (Landis und Koch 1977). Demgegenüber standen 0,32 (95\%-KI [0,23,

ACA

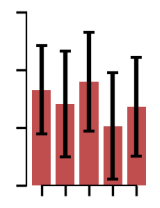

MCA

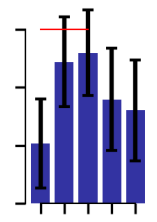

ICA

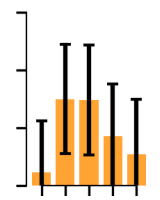

Rater A (unerfahren)

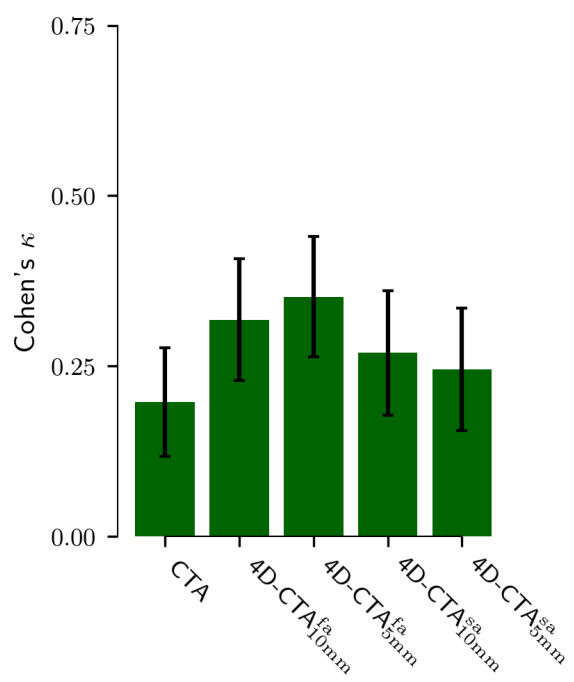

PA

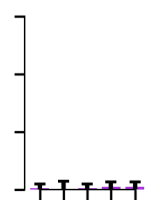

BA

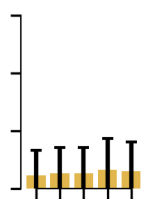

Rater B (erfahren)

ACA

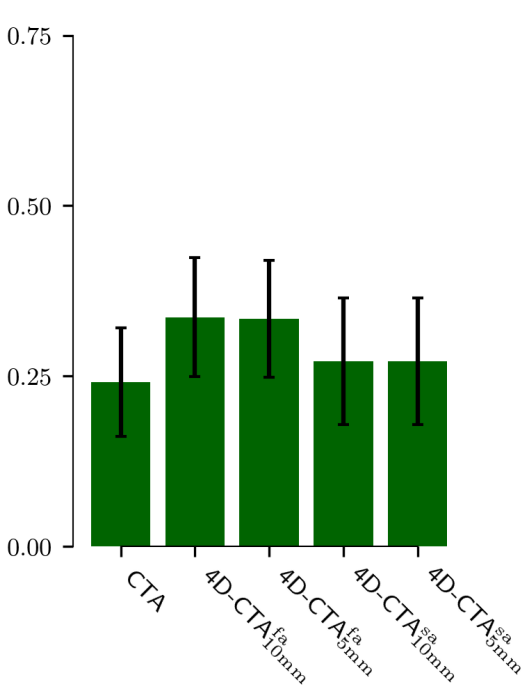

BA

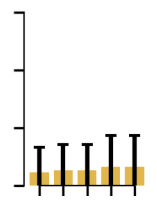

PA

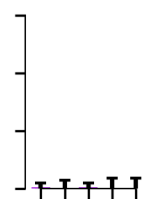

ICA

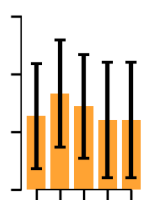

Abbildung 13: Beurteilung des Vasospasmusgrades (Interrater-Reliabilität). Die Güte der Beurteilung wird hier quantifiziert durch die Interrater-Reliabilität (Cohen's к) zwischen DSA als Goldstandard und CTA bzw. 4D-CTA der einzelnen Rater. Die Ergebnisse sind dargestellt für Rater A (links) und Rater B (rechts) über alle Gefäße (grün) und über die einzelnen betrachteten Gefäße einzeln aufgeschlüsselt. Rote Balken zeigen signifikante Unterschiede (5\%-Konfidenzniveau). 
0,41]) bzw. 0,35 (95\%-KI [0,26, 0,44]) für die früharterielle 4D-CTA bei $10 \mathrm{~mm}$ bzw. $5 \mathrm{~mm}$ Schichtdicke. Die Unterschiede zur CTA waren also statistisch nicht signifikant. Dennoch waren beide Werte immerhin als ausreichende Übereinstimmung einzustufen (Landis und Koch 1977). Für die spätarterielle 4D-CTA ergaben sich etwas kleinere Werte von 0,27 (95\%-KI [0,18, 0,36]) bzw. 0,24 (95\%-KI [0,15, 0,33]) für $10 \mathrm{~mm}$ bzw. $5 \mathrm{~mm}$ Schichtdicke, die beide gerade noch als ausreichende Übereinstimmung eingestuft werden. Auch diese Unterschiede waren nicht signifikant zur konventionellen CTA.

Für den erfahrenen Rater B zeigten sich völlig analoge Ergebnisse, ohne hier alle Zahlenwerte zu nennen. Die früharterielle 4D-CTA erreichte die beste Übereinstimmung mit dem Goldstandard DSA. Hierbei wurde beobachtet, dass bei der Bewertung der unerfahrene und der erfahrene Rater mit allen Methoden in etwa ähnliche Übereinstimmungen mit dem Goldstandard erreichten.

Für die einzelnen Gefäße waren die Ergebnisse für MCA und ICA mit den obigen Resultaten über alle Gefäße konsistent, auch wenn die absoluten Zahlen etwas abwichen. Bei ACA, PA und BA waren die Ergebnisse etwas unübersichtlicher, es lässt sich aber festhalten, dass (1) kein signifikanter Unterschied zwischen irgendeiner Kombination von Methoden gefunden wurde und (2) außer eine Ausnahme (ACA, Rater A) die Übereinstimmungen mit dem Goldstandard für die früharterielle 4D-CTA grundsätzlich höher als für die konventionelle CTA waren.

\subsubsection{Pearson-Korrelation}

Eine alternative Quantifizierung der Übereinstimmung von CTA bzw. 4D-CTA gegenüber der DSA als Goldstandard ist durch den PearsonKorrelationskoeffizienten gegeben. Die Ergebnisse sind in Abbildung 14 dargestellt. Über alle Gefäße erreichte der unerfahrene Rater A mit der CTA eine Korrelation von 0,49 (95\%-KI [0,38, 0,59]). Demgegenüber standen höhere Werte von 0,68 (95\%-KI [0,60, 0,75]) bzw. 0,65 (95\%-KI $[0,56,0,72])$ für die früharterielle 4D-CTA bei $10 \mathrm{~mm}$ bzw. $5 \mathrm{~mm}$ Schichtdicke. Hierbei war der Unterschied zwischen CTA und $4 \mathrm{D}-\mathrm{CTA}_{\mathrm{fa}, 10 \mathrm{~mm}}$ statistisch signifikant. Für die spätarterielle 4DCTA ergaben sich Werte zwischen denen von CTA und früharterieller 4D-CTA: 0,59 (95\%-KI [0,49, 0,68]) bzw. 0,60 (95\%-KI [0,50, 0,68]) 


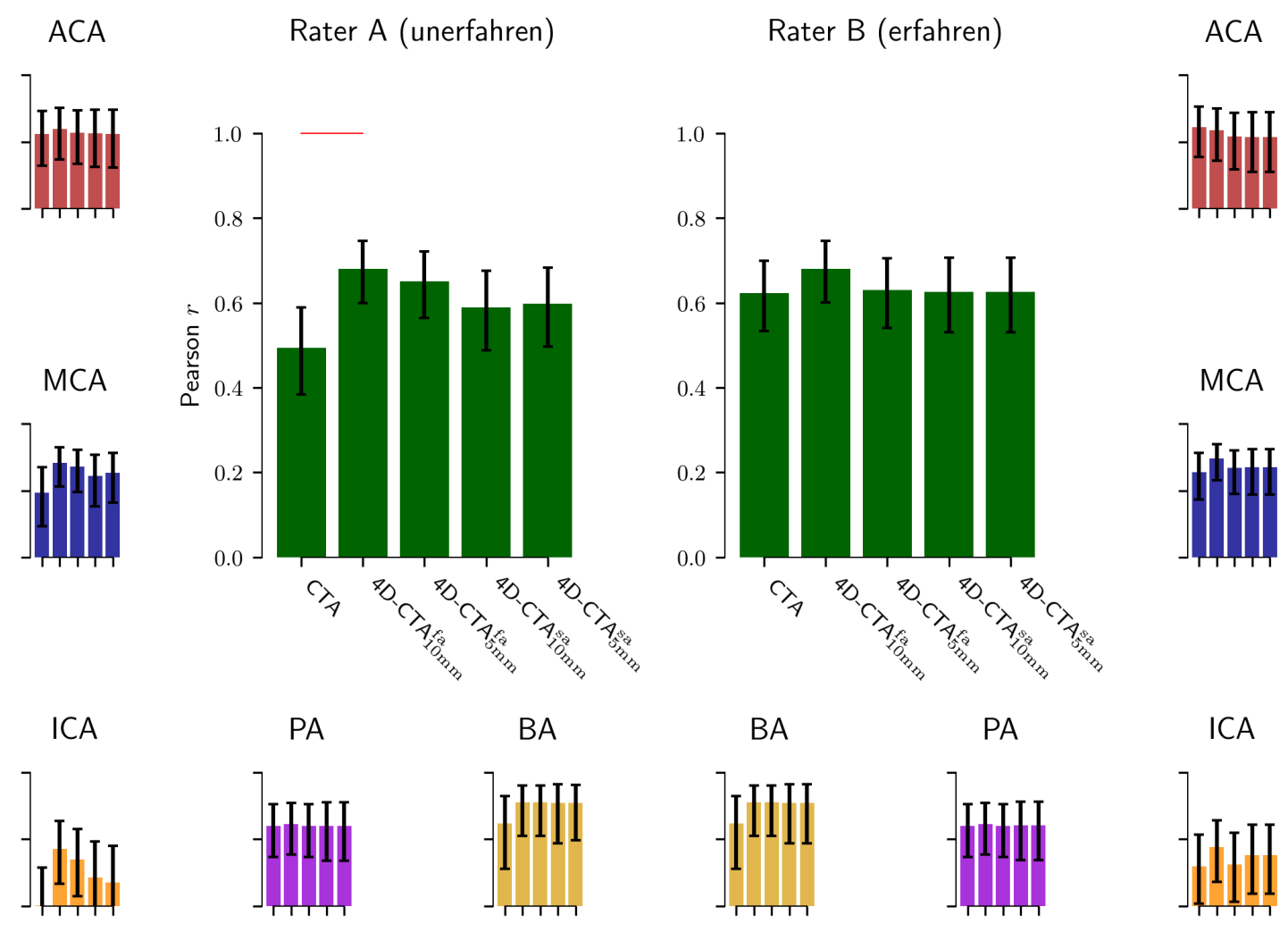

Abbildung 14: Beurteilung des Vasospasmusgrades (PearsonKorrelation). Die Güte der Beurteilung wird hier quantifiziert durch die Korrelation (Pearson $r$ ) zwischen DSA als Goldstandard und CTA bzw. 4D-CTA. Die Ergebnisse sind dargestellt für Rater A (links) und Rater B (rechts) über alle Gefäße (grün) und über die einzelnen betrachteten Gefäße einzeln aufgeschlüsselt. Rote Balken zeigen signifikante Unterschiede (5\%-Konfidenzniveau).

für $10 \mathrm{~mm}$ bzw. $5 \mathrm{~mm}$ Schichtdicke. Diese Unterschiede waren nicht signifikant zur konventionellen CTA.

Der erfahrene Rater B erreichte mit CTA, 4D-CTA $\mathrm{fa}_{\mathrm{fa}, 5 \mathrm{~mm}}$ und der spätarteriellen 4D-CTA fast identische Korrelationen mit dem Goldstandard DSA: 0,62 (95\%-KI [0,53, 0,70]) für die CTA bzw. 0,63 (95\%KI $[0,54,0,70])$ für alle drei 4D-CTA-Verfahren. Lediglich die 4D$\mathrm{CTA}_{\mathrm{fa}, 10 \mathrm{~mm}}$ erreichte eine höhere Übereinstimmung mit dem Goldstandard DSA bei einer Korrelation von 0,68 (95\%-KI [0,60, 0,75]). Auch hier zeigte sich, dass bei der Bewertung der unerfahrene und 
der erfahrene Rater mit allen Methoden in etwa ähnliche Übereinstimmungen mit dem Goldstandard erreichten.

Für die einzelnen Gefäße waren die Ergebnisse für MCA und ICA mit den obigen Resultaten über alle Gefäße größtenteils konsistent, auch wenn die absoluten Zahlen etwas abwichen. Auch bei der ACA war das Ergebnis für Rater A hier konsistent. Lediglich der erfahrene Rater B erreichte mit der CTA für dieses Gefäß eine höhere Übereinstimmung als mit der 4D-CTA. Allerdings war die Effektstärke gering und der Unterschied statistisch nicht signifikant.

Für die PA erreichten beide Rater mit allen Verfahren ähnliche Übereinstimmungen mit der DSA. Für die BA war die Übereinstimmung für beide Rater für jedes der 4D-CTA-Verfahren höher als für die konventionelle CTA, jedoch nicht signifikant.

\subsection{Interrater-Reliabilität der Vasospasmus- detektion}

Neben der bloßen Sensitivität und Spezifität, die die Detektionswahrscheinlichkeiten eines Vasospasmus für beide Rater unabhängig voneinander beschreiben, wurde auch untersucht, inwieweit die beiden Rater mit den verschiedenen Verfahren in dieser Detektion übereinstimmten. Hierzu wurden zwei statistische Maße herangezogen: die Interrater-Reliabilität nach Cohen sowie die Korrelation nach Pearson (siehe Abschnitt 2.5.3).

Für beide Maße ergab sich ein konsistentes Bild. Über alle Gefäße zeigte die CTA nur eine moderate Übereinstimmung der beiden Rater. Im Vergleich dazu lieferte jedes 4D-CTA-Verfahren eine signifikant bessere und fast perfekte Übereinstimmung. Der früharterielle Zeitpunkt der Rekonstruktion war dem spätarteriellen tendenziell überlegen. Einzig für die ACA konnte auch mit der CTA eine bessere Übereinstimmung der Rater erreicht werden, sodass die 4D-CTA hier nur zu einer geringen Verbesserung führte. Im Folgenden werden alle Ergebnisse detailliert aufgeschlüsselt. 

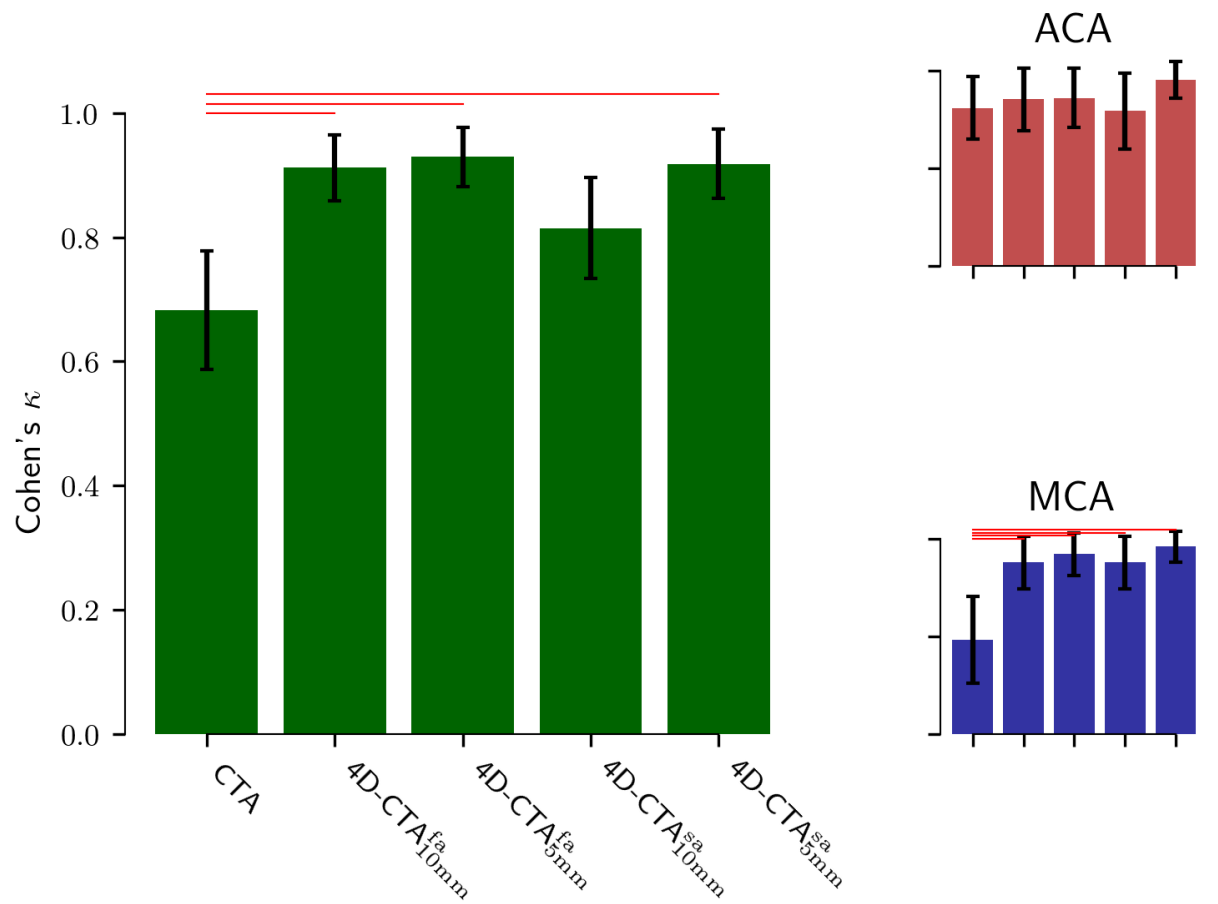

BA

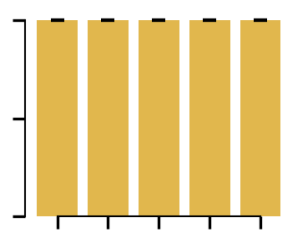

PA

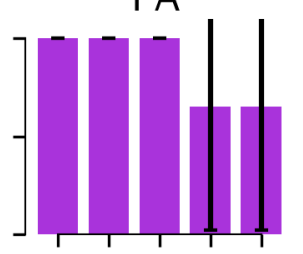

ICA

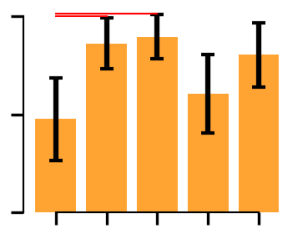

\begin{abstract}
Abbildung 15: Interrater-Reliabilität der Vasospasmusdetektion. Interrater-Reliabilität, gemessen durch Cohen's $\kappa$ zwischen Rater A und Rater B für alle Verfahren über alle Gefäße (grün) und über die einzelnen betrachteten Gefäße einzeln aufgeschlüsselt. Rote Balken zeigen signifikante Unterschiede (5\%-Konfidenznivaus).
\end{abstract}

\title{
3.11.1 Cohens's $k$
}

Die Ergebnisse sind in Abbildung 15 dargestellt. Für die konventionelle CTA ergab sich eine Interrater-Reliabilität, gemessen durch Cohens's $\kappa$, von 0,68 (95\%-KI [0,59, 0,78]). Diese Übereinstimmung der beiden Rater ist nur als moderat einzustufen (Landis und Koch 1977). Demgegenüber stand eine fast perfekte Übereinstimmung von $0,91(95 \%-K I[0,86,0,97])$ für die $4 \mathrm{D}-\mathrm{CTA}_{\mathrm{fa}, 10 \mathrm{~mm}} \mathrm{bzw}$. von 0,93 (95\%-KI $[0,88,0,98])$ für die $4 \mathrm{D}-\mathrm{CTA}_{\mathrm{fa}, 5 \mathrm{~mm}}$. Auch die spätarterielle $4 \mathrm{D}$-CTA lie- 
ferte immer fast perfekte Übereinstimmungen von 0,81 (95\%-KI $[0,86,0,97])$ bzw. 0,92 (95\%-KI [0,86, 0,97]). Hierbei waren die Unterschiede zwischen CTA und allen 4D-CTA-Verfahren mit Ausnahme der spätarteriellen 4D-CTA bei einer Schichtdicke von $10 \mathrm{~mm}$ auch statistisch signifikant.

Bei Betrachtung der einzelnen Arterien zeigte sich ein analoges Resultat sowohl für MCA als auch ICA. In beiden Arterien zeigte die 4DCTA bei Verwendung der früharteriellen 4D-CTA eine signifikante Verbesserung der Interrater-Reliabilität. Bei der ACA war schon die
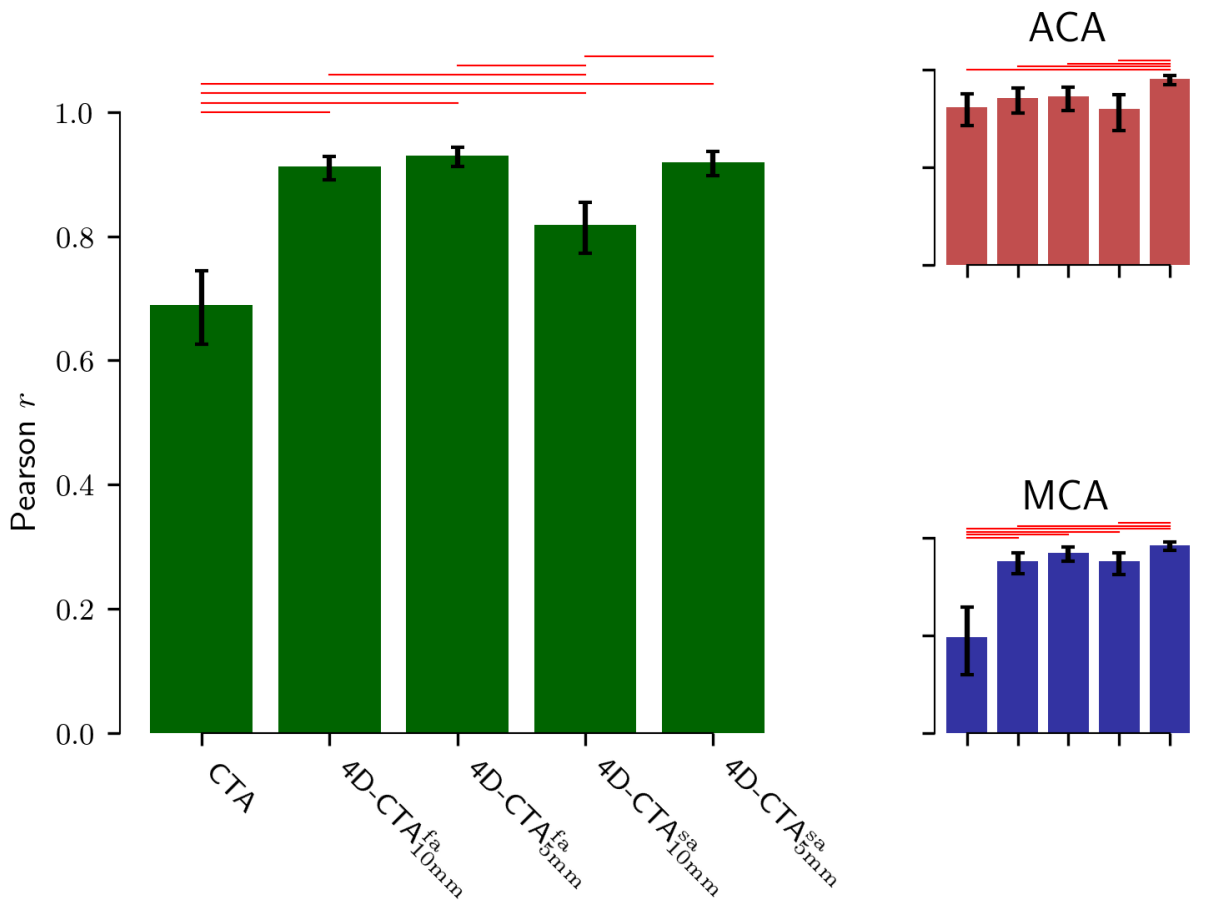

BA

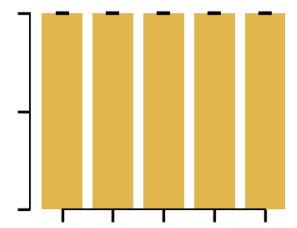

PA

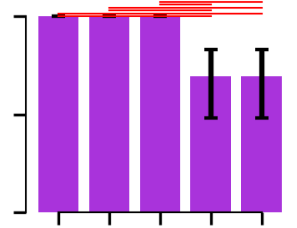

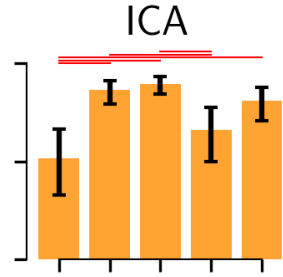

Abbildung 16: Interrater-Korrelation der Vasospasmusdetektion. Interrater-Reliabilität, gemessen durch Pearson's Korrelationskoeffizienten $r$ zwischen Rater A und Rater B für alle Verfahren über alle Gefäße (grün) und über die einzelnen betrachteten Gefäße einzeln aufgeschlüsselt. Rote Balken zeigen signifikante Unterschiede (5\%-Konfidenz). 
Interrater-Reliabilität der CTA etwas höher $(0,81 ; 95 \%-K I \quad[0,65$, 0,97]), sodass nur eine mäßige und nicht signifikante Steigerung durch die 4D-CTA erreicht wurde. Bei BA und PA zeigte sich wie schon zuvor beobachtet aufgrund der kleinen Stichprobengröße ein weniger aussagekräftiges Ergebnis.

\subsubsection{Pearson-Korrelation}

Die Ergebnisse sind in Abbildung 16 dargestellt. Die Quantifizierung der Interrater-Reliabilität durch deren Korrelationskoeffizienten (Pearson $r$ ) bestätigte die Resultate von Cohen's $\kappa$. Einer guten Korrelation zwischen den Ratern von 0,69 (95\%-KI [0,63, 0,74]) für die CTA stand für jedes der 4D-CTA-Verfahren eine sehr gute und im Vergleich zur CTA signifikant höhere Korrelation gegenüber. Diese betrugen 0,91 (95\%-KI [0,89, 0,93]) bzw. 0,93 (95\%-KI [0,91, 0,94]) für die früharterielle 4D-CTA und 0,82 (95\%-KI [0,77, 0,85]) bzw. 0,92 (95\%-KI $[0,90,0,94])$ für die spätarterielle 4D-CTA.

Auch hier bestätigten sich die schon zuvor beobachteten Trends für die einzelnen Arterien. Wieder erreichten die Rater mit der CTA speziell für die ACA eine höhere Korrelation von 0,80 (95\%-KI [0,71, $0,88]$ ) als über alle Arterien. Daher waren die mit der 4D-CTA erreichten Steigerungen der Korrelation hier moderater. Die beste Korrelation $(0,95,95 \%-K I[0,92,0,97])$ erreichte hier die $4 \mathrm{D}-\mathrm{CTA}_{\mathrm{sa}, 5 \mathrm{~mm}}$, die signifikant besser als alle anderen Verfahren war. Dieses Ergebnis weicht vom bisher beobachteten Trend, dass die früharterielle 4D-CTA die verlässlichste Bewertung boten, punktuell ab. Die Ergebnisse von MCA und ICA verhielten sich analog zu den Resultaten über alle Gefäße. Bei BA und PA zeigte sich wie schon zuvor beobachtet aufgrund der kleinen Stichprobengröße ein wenig aussagekräftiges Ergebnis.

\subsection{Interrater-Reliabilität des Vasospasmusgrades}

Neben der unabhängigen Bewertung des Vasospasmusgrades wurde auch untersucht, inwieweit die beiden Rater mit den verschiedenen Verfahren bei dieser Bewertung übereinstimmten. Hierzu wurden zwei statistische Maße herangezogen: die Interrater-Reliabilität nach Cohen, sowie die Korrelation nach Pearson (siehe Abschnitt 2.5.4). 
Für beide Maße ergab sich ein konsistentes Bild, welches ferner konsistent mit den Ergebnissen in Abschnitt 3.11 ist. Über alle Gefäße zeigte die CTA nur eine moderate Übereinstimmung der beiden Rater. Im Vergleich dazu lieferte jedes 4D-CTA signifikant bessere und substantielle Übereinstimmung. Der früharterielle Zeitpunkt der Rekonstruktion war dem spätarteriellen tendenziell überlegen. Einzig für die ACA konnte auch mit der CTA eine bessere Übereinstimmung der Rater erreicht werden, sodass die 4D-CTA hier nur zu einer geringen Verbesserung führt. Im Folgenden werden alle Ergebnisse detailliert aufgeschlüsselt.

\subsubsection{Cohen's $k$}

Die Ergebnisse sind in Abbildung 17 dargestellt. Für die konventionelle CTA ergab sich eine Interrater-Reliabilität, gemessen durch Cohens's $\kappa$, von 0,56 (95\%-KI [0,46, 0,65]). Diese Übereinstimmung der beiden Rater ist nur als moderat einzustufen (Landis und Koch 1977). Demgegenüber stand eine fast perfekte Übereinstimmung von 0,88 (95\%-KI $[0,83,0,94])$ für die $4 \mathrm{D}-\mathrm{CTA}_{\mathrm{fa}, 10 \mathrm{~mm}} \mathrm{bzw}$. von $0,89(95 \%-\mathrm{KI}$ $[0,84,0,94])$ für die $4 \mathrm{D}-\mathrm{CTA}_{\mathrm{fa}, 5 \mathrm{~mm}}$. Auch das spätarterielle Verfahren lieferte immer noch substantielle Übereinstimmungen von 0,72 (95\%-KI $[0,64,0,80])$ bzw. 0,80 (95\%-KI $[0,74,0,88])$. Hierbei waren die Unterschiede zwischen CTA und allen 4D-CTA-Verfahren mit Ausnahme des spätarteriellen Verfahrens bei einer Schichtdicke von 10 $\mathrm{mm}$ auch statistisch signifikant.

Bei Betrachtung der einzelnen Arterien zeigte sich ein analoges Resultat sowohl für MCA als auch ICA. In beiden Arterien zeigte die früharterielle 4D-CTA eine signifikante Verbesserung der InterraterReliabilität. Bei der ACA war schon die Interrater-Reliabilität der CTA etwas höher $(0,61 ; 95 \%$-KI $[0,45,0,78])$, sodass nur eine mäßige und nicht signifikante Steigerung durch die 4D-CTA erreicht wurde. Bei BA und PA zeigte sich wie schon zuvor beobachtet aufgrund der kleinen Stichprobengröße ein wenig aussagekräftiges Ergebnis. 

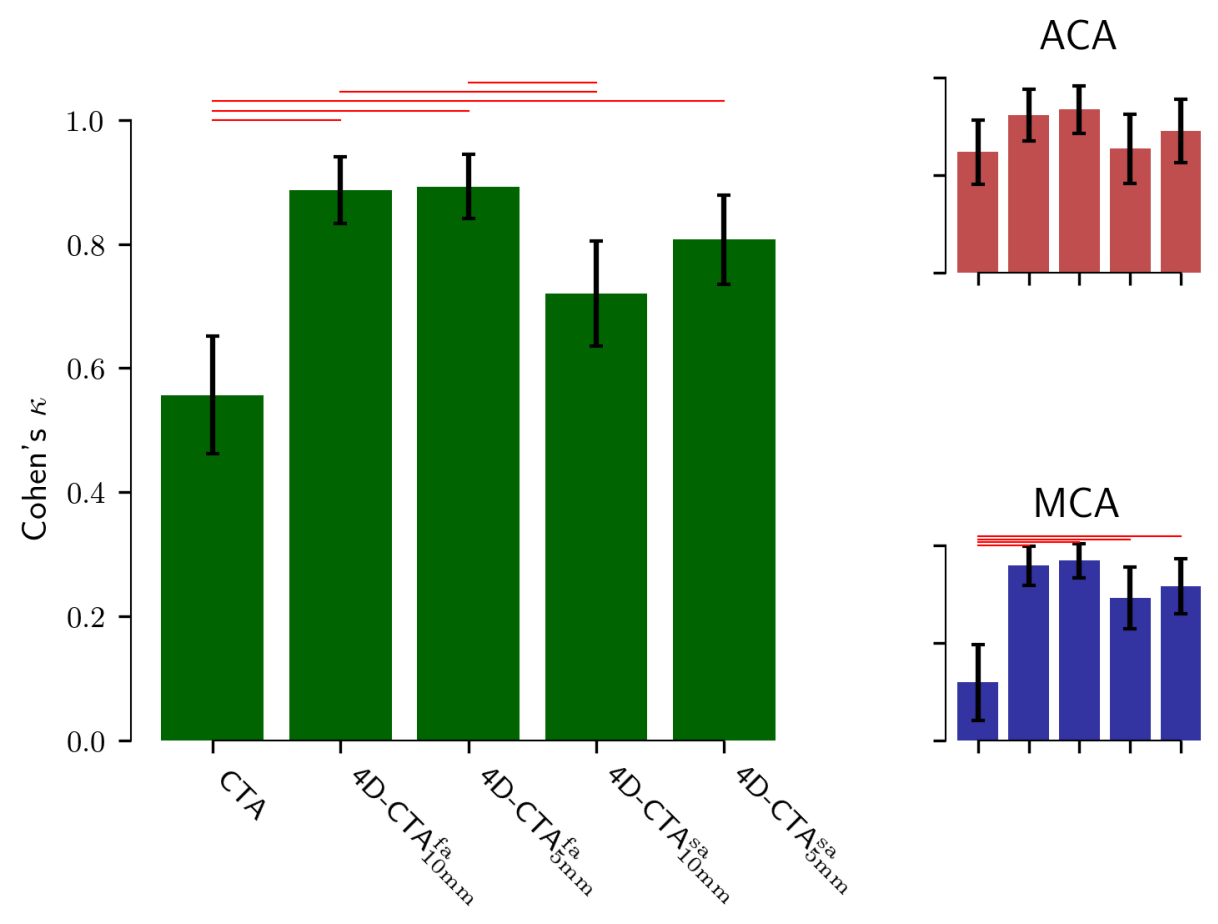

BA

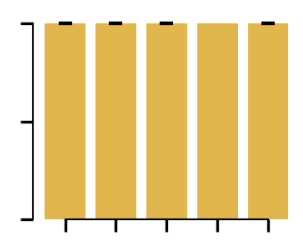

PA

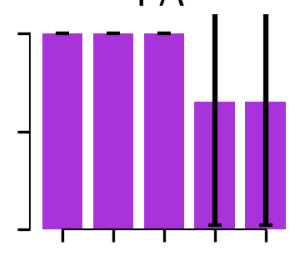

ICA

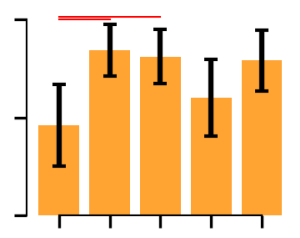

\begin{abstract}
Abbildung 17: Interrater-Relibilität der Beurteilung des Vasospasmusgrades zwischen Rater A und B. Interrater-Reliabilität, gemessen durch Cohen's к zwischen Rater A und Rater B. Ergebnisse sind dargestellt für alle Verfahren über alle Gefäße (grün) und über die einzelnen betrachteten Gefäße einzeln aufgeschlüsselt. Rote Balken zeigen signifikante Unterschiede (5\%-Konfidenz).
\end{abstract}

\title{
3.12.2 Pearson-Korrelation
}

Die Ergebnisse sind in Abbildung 18 dargestellt. Die Quantifizierung der Interrater-Reliabilität durch deren Korrelationskoeffizienten (Pearson $r$ ) bestätigte die Resultate von Cohen's $\kappa$. Einer guten Korrelation zwischen den Ratern von 0,74 (95\%-KI [0,69, 0,79]) für die CTA stand für jedes der 4D-CTA-Verfahren eine sehr gute und im Ver- 
gleich zur CTA signifikant höhere Korrelation gegenüber. Diese betrugen 0,94 (95\%-KI [0,93, 0,95]) bzw. 0,95 (95\%-KI [0,93, 0,96]) für die früharterielle 4D-CTA und 0,86 (95\%-KI [0,82, 0,89]) bzw. 0,91 $(95 \%-K I[0,88,0,93])$ für das spätarterielle Verfahren.

Auch hier bestätigten sich die schon zuvor beobachteten Trends für die einzelnen Arterien. Wieder erreichten die Rater mit der CTA speziell für die ACA eine höhere Korrelation von 0,80 (95\%-KI [0,71, $0,88]$ ) als über eine alle Arterien. Daher waren die mit der 4D-CTA
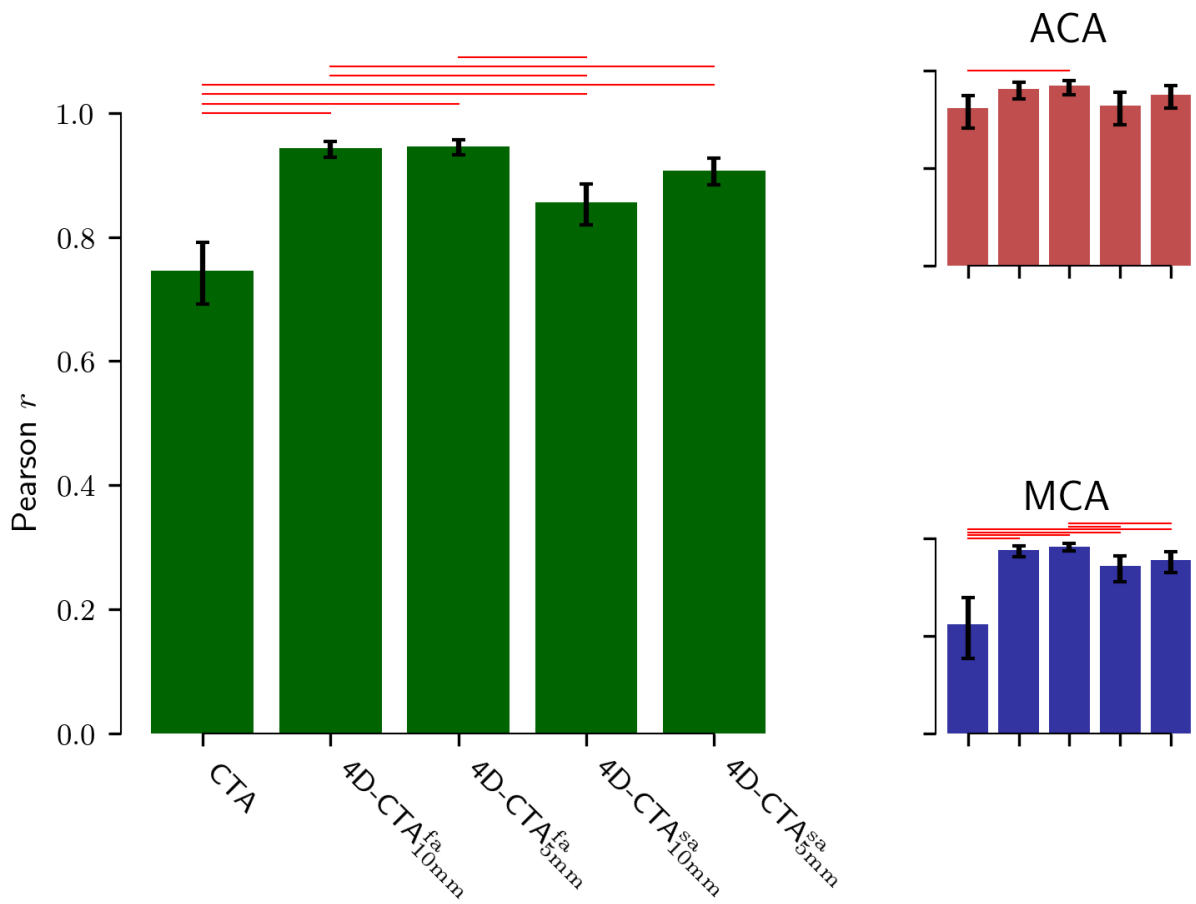

BA

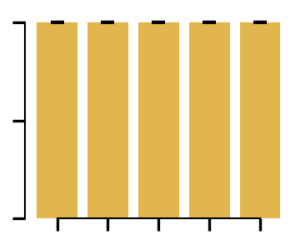

PA

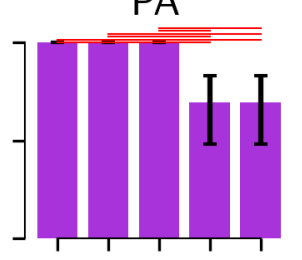

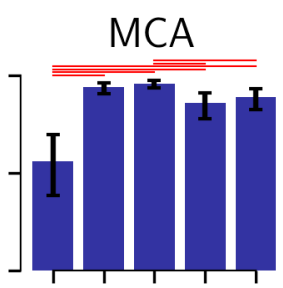

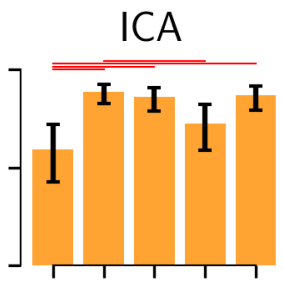

Abbildung 18: Korrelation der Beurteilung des Vasospasmusgrades zwischen Rater $A$ und B. InterraterReliabilität, gemessen durch die Pearson's Korrelationskoeffizienten $r$ zwischen Rater A und Rater B. Ergebnisse sind dargestellt für alle Verfahren über alle Gefäße (grün) und über die einzelnen betrachteten Gefäße einzeln aufgeschlüsselt. Rote Balken zeigen signifikante Unterschiede (5\%-Konfidenzniveau). 
erreichten Steigerungen der Korrelation hier moderater. Die beste Korrelation $(0,95,95 \%-K I[0,92,0,97])$ erreichte hier die $4 \mathrm{D}-\mathrm{CTA}_{\mathrm{fa}, 5 \mathrm{~mm}}$. Die Ergebnisse für MCA und ICA verhielten sich exakt analog zu den Resultaten über alle Gefäße. Bei BA und PA zeigte sich wie schon zuvor beobachtet aufgrund der kleinen Stichprobengröße ein wenig aussagekräftiges Ergebnis. 


\section{Diskussion}

\subsection{Patientenkollektiv}

Trotz der kleinen Stichprobengröße des betrachteten Patientenkollektivs ergab sich eine gute Übereinstimmung mit der aktuellen Studienlage zur Epidemiologie der SAB und Vasospasmen. Dies zeigte sich für Geschlechterverteilungen, Altersverteilungen, Aneurysmenlokalisation und die zeitliche Auftrittsverteilung von interventionell behandlungsbedürftigen Vasospasmen nach SAB. Daher kann die Studie trotz der limitierten Stichprobengröße als repräsentativ erachtet werden.

\subsubsection{Demographie SAB}

Die Geschlechterverteilung des betrachteten Patientenkollektivs entspricht der aktuellen Studienlage. Bei der Auswahl des SAB-Patientenkollektivs zeigte sich ein 1,56-faches Überwiegen des weiblichen Geschlechts (61\% Frauen und 39\% Männer). Dies deckt sich mit den Ergebnissen von Linn et al. (1996), die für Frauen ein 1,6-fach höheres Risiko für eine SAB gezeigt haben. Auch nach de Rooij et al. (2007) ist die Inzidenz einer SAB bei Frauen höher, betrachtet man eine Altersgruppe von 55-85-Jährigen. Bei einer jüngeren Altersgruppe (25-45 Jahre) hingegen fand diese Studie eine signifikant höhere Inzidenz bei Männern. Die Gründe für das Überwiegen des weiblichen Geschlechts bei SAB sind nicht eindeutig geklärt. Hormonelle Faktoren scheinen eine mögliche Erklärung zu sein. Dies wird auch durch das Ansteigen der weiblichen Inzidenz mit zunehmendem Alter unterstützt (Mhurchu et al. 2011; Longstreth 1994; de Rooij et al. 2007).

Auch die Altersverteilung der im Rahmen dieser Promotion untersuchten SAB-Patienten deckt sich mit aktuellen epidemiologischen Daten zur SAB. Es ergab sich ein medianes Alter von 54 Jahren und ein mittleres Alter von 55 Jahren. Dies entspricht den Ergebnisse von zahlreichen vorherigen Studien. Die Metastudie von Hop et al. (1997), die die Resultate von 21 Studien zusammenfasste, fand das 
gleiche mittlere Alter von 55 Jahren. Diese Resultate wurden später auch durch die Ergebnisse von van Gijn et al. (2007), demzufolge die Hälfte der SAB-Patienten zum Zeitpunkt der Aneurysma-Ruptur jünger als 55 Jahre ist, sowie von Mees et al. (2007), da Costa et al. (2005), und Rinkel et al. (1998) bestätigt.

\subsubsection{Vasospasmusinzidenz nach SAB}

Von dem betrachteten Patientenkollektiv mit SAB nach rupturiertem Aneurysma entwickelten 28,7\% im Verlauf einen interventionell behandlungsbedürftigen Vasospasmus. Dorsch und King (1994) führen in einem umfassenden Literaturüberblick zu Vasospasmen nach SAB bei rupturierten Aneurysma in mehr als 30000 Fällen an, dass die Inzidenz eines angiographisch sichtbaren Vasospasmus 43,3\% und die eines symptomatischen Vasospasmus oder eines Delayed ischaemic Deficits 32,5\% sei. Hierbei muss berücksichtigt werden, dass sowohl die begriffliche Vielfalt und z.T. uneindeutige Verwendung als auch die Breite der betrachteten Zeitspanne die Aussagekraft der „Literatur" limitieren (Dorsch und King 1994). Unter diesen Gesichtspunkten kann festgestellt werden, dass die Inzidenz eines DCIs bei dem von uns betrachteten Patientenkollektiv in guter Näherung mit den Literaturangaben übereinstimmt.

\subsubsection{Lokalisation der für die SAB ursächlichen Aneurysma}

Die Häufigkeit der Aneurysma-Lokalisation, deren Ruptur für die SAB dieser Studie verantwortlich war, deckt sich ebenfalls mit der gängigen Literatur. Die Ruptur eines Aneurysmas der vorderen Zirkulation macht je nach Studie bis zu 94,4\% als Ursache für eine SAB aus (da Costa et al. 2005; Spendel 2008). Nach Spendel (2008) seien mit 40\% Aneurysmen am häufigsten in der ACOM oder ACA. Am zweithäufigsten sei die ICA mit $30 \%$, bei $20 \%$ sei die MCA betroffen und 10\% würden Aneurysmen der A. basilaris und der A. vertebralis ausmachen.

Bei dem in dieser Studie ausgewählten Patientenkollektiv waren $40 \%$ der ursächlichen Aneurysma in der ACOM lokalisiert, ACA-Aneurysmen gab es bei $4 \%$, MCA-Aneurysma bei $15 \%$, ICA-Aneurysma bei $13 \%$, Aneurysma von BA und A. vertebralis zusammengefasst bei $14 \%$. Auffällig ist also ein prozentual niedriger Anteil der ICA-Aneu- 
rysma. Aufgrund der limitierten Anzahl von 85 Patienten gegenüber einer Anzahl von zehn untersuchten Arterien ist eine statistische Abweichung wahrscheinlich.

\subsubsection{Behandlungsmethode der SAB}

Sowohl die Coil-Embolisation als auch das Clipping sind etablierte Verfahren zur Ausschaltung von intrakraniellen Aneurysmen. Die Entscheidung wird in der Regel interdisziplinär von Neuradiologen, Neurologen und Neurochirurgen individuell für jeden Patienten getroffen. Kriterien stellen u. A. die Lokalisation, die Größe, die Konfiguration des Aneurysmas und das Alter des Patienten dar (Zhao et al. 2017). So besteht bei Patienten in höherem Lebensalter häufig auch ein erhöhtes Operationsrisiko (Sedat et al. 2002). Aneurysmen des hinteren Kreislaufs werden ebenfalls wegen der schlechten chirurgischen Zugänglichkeit häufiger interventionell behandelt ( $\mathrm{Li}$ et al. 2013) .

Die am häufigsten durchgeführte Behandlungsmethode bei rupturiertem Aneurysma war bei dem in dieser Studie ausgewählten Patientenkollektiv mit insgesamt 53\% das Coiling. Bei 45,1\% der Patienten wurde das Aneurysma geclippt. Ein Vergleich mit der Literatur zeigt, dass zwischen 50\% und 85\% aller Aneurysmen endovaskulär mittels Coil-Embolisation behandelt werden (Petridis et al. 2017).

\subsubsection{Vasospasmus und vasospasmusbedingte Infarkte}

Bei dem Patientenkollektiv dieser Studie, die einen Vasospasmus nach SAB bekamen, entwickelten $45 \%$ einen vasospasmusbedingten Infarkt im Verlauf, 55\% entwickelten keinen Infarkt, der mit dem Vasospasmus assoziiert war.

Im Folgenden wird die Abhängigkeit von der Behandlungsmethode diskutiert. Hierfür werden die beiden Patienten, die sowohl ein Coiling als auch ein Clipping des Aneurysmas erhalten haben, ausgenommen, da hinsichtlich der Komplikationen keine eindeutige Zuordnung zur Behandlungsmethode möglich ist. Dadurch sind die Zahlen im Folgenden etwas verändert im Vergleich zu Abschnitt 3.6.

Von den 38\% (11 von 29) Patienten mit Vasospasmus und Coil-Embolisation des Aneurysmas entwickelten 55\% (6 von 11) im Verlauf ei- 
nen Infarkt im Vasospasmusgebiet. 62\% (18 von 29) der Patienten mit einem interventionell behandlungsbedürftigen Vasospasmus hatten vorab ein Clipping des rupturierten Aneurysmas erhalten. 39\% (7 von 18) dieser geclippten Vasospasmuspatienten erlitten einen Infarkt im Verlauf. Da das Clipping mit 45\% (im Vergleich zu 55\% CoilEmbolisationen, siehe Abschnitt 3.6) die weniger häufig eingesetzte Behandlungsmethode bei SAB nach Aneurysmaruptur darstellte, aber trotzdem bei dem Großteil von 62\% der interventionell behandlungsbedürftigen Vasospasmen nach Clipping auftrat, lässt sich schließen, dass das Clipping eine etwas schlechtere Prognose als das Coiling bezüglich des Auftretens von Vasospasmen hat.

Dieses Resultat deckt sich größtenteils mit der Literatur, auch wenn der Einfluss der Behandlungsmethode auf die Folgekomplikationen kontrovers diskutiert wird. So kommen sowohl Charpentier et al. (1999), als auch Hoh et al. (2004) in ihren Studien zu dem Ergebnis, dass die Behandlungsmethode des rupturierten Aneurysmas keinen Einfluss auf die Entwicklung eines Vasospasmus hat. Die Metaanalyse von Li et al. (2013) kam dagegen zu dem Schluss, dass sowohl DCI als auch Vasospasmen häufiger nach Clipping als nach einer Coil-Embolisation vorzufinden sind. Bezüglich des Endpunktes eines ischämischen Infarktes als Komplikation einer SAB spiele die Behandlungsmethode allerdings keine Rolle.

Auch Hohlrieder et al. (2002) kommen in ihrer Studie zu dem Ergebnis, dass Vasospasmen nach Clipping signifikant häufiger auftreten als nach Coiling (70\% vs. 44\%). Dies gilt ebenfalls für das Auftreten von ischämischen Infarkten (62\% nach Clipping vs. $48 \%$ nach Coiling). Nach dieser Studie entwickelten insgesamt 53,5\% beider Behandlungsmethoden zusammengenommen einen interventionspflichtigen Vasospasmus.

Eine Studie des international subarachnoid aneurysm trial (ISAT-Studie) hat gezeigt, dass die Wahrscheinlichkeit, fünf, zehn und 18 Jahre nach der SAB ein behinderungsfreies Leben zu führen, nach endovaskulärer Behandlungsmethode signifikant höher war als bei neurochirurgischer Behandlung des Aneurysmas (Molyneux et al. 2005; Molyneux et al. 2009; Molyneux et al. 2015). Jedoch bestehen zwei Einschränkungen: (1) Die endgültige Aussagekraft der ISAT-Studie wurde aufgrund mehrerer möglicher Einschränkungen kontrovers 
diskutiert, darunter der Ausschluss eine Großteils der Patienten von der Randomisierung sowie unterschiedliche Anforderungen an die Erfahrung der zur Studie zugelassenen Ärzte. Dennoch werden die Ergebnisse der Studie im Allgemeinen anerkannt und dem Behandlungsprotokoll zugrunde gelegt (Raper und Allan 2010). (2) Die Studie ist nur bedingt mit dieser Promotion vergleichbar, da Überleben und Behinderungsfreiheit nur indirekte Indikatoren für vasospasmusbedingte Infarkte ist. Vasospasmusbedingte Infarkte können selbstverständlich $\mathrm{zu}$ unterschiedlichen Ausgängen führen, und andere Komplikationen nach SABs können für Behinderung verantwortlich sein. Trotzdem unterstreicht diese große Studie von Molyneux et al. (2015), dass es im Verlauf der neurochirurgischen Behandlung häufiger zu Komplikationen kommt (Molyneux et al. 2015; Petridis et al. 2017).

Zusammenfassend kann zu dem Patientenkollektiv, das im Rahmen dieser Promotion untersucht wurde, gesagt werden, dass die Größe der Studie zwar eine Limitation zur Verallgemeinerung der hier gefundenen Ergebnisse ist. Nichtsdestotrotz zeigt ein Vergleich mit der Literatur, dass das untersuchte Patientenkollektiv als repräsentativ $\mathrm{zu}$ erachten ist.

\subsection{Untersuchungsmethodik}

Die grundlegende Hypothese, die in dieser Arbeit untersucht wurde, kann klar bestätigt werden. Die aus der CTP rekonstruierte 4D-CTA könnte die konventionelle CTA vollständig ersetzen. Sie erreichte konsistent mindestens gleichwertige Ergebnisse hinsichtlich der diagnostischen Güte. In keinem der untersuchten Marker erzielte eines der 4D-CTA-Verfahren signifikant schlechtere Ergebnisse als die konventionelle CTA. Dies lässt den Schluss zu, dass die 4D-CTA mindestens gleichwertige Ergebnisse im Vergleich zur konventionellen CTA erzielt, sowohl bei der Diagnose von Vasospasmen als auch bei der Bewertung des Schweregrades. Auf die konventionelle CTA könnte somit in der Untersuchungsroutine nach SAB verzichtet werden.

Unter den verschiedenen Rekonstruktionsmethoden ist die früharterielle 4D-CTA der spätarteriellen 4D-CTA klar vorzuziehen. Die früharterielle 4D-CTA erzielte in keinem der betrachteten Fälle (mit Aus- 
nahme der Spezifität des unerfahrenen Raters, siehe Abschnitt 3.9.2) schlechtere Ergebnisse als die konventionelle CTA, unabhängig von deren Signifikanz. Bei Verwendung dieser Methode bietet die 4DCTA gegenüber der konventionellen CTA sogar einen diagnostischen Mehrwert, der insbesondere unerfahrenen Ärzten und Ärztinnen nutzen könnte.

\subsubsection{Diagnostischer Mehrwert durch 4D-CTA}

Die Anwendung von Rekonstruktionen aus einer 4D-CTA bedeutet insbesondere für unerfahrene Ärztinnen und Ärzte einen diagnostischen Mehrwert. Denn über die Gleichwertigkeit hinaus erzielte die 4D-CTA bei Verwendung der früharteriellen Serie sogar konsistent bessere Ergebnisse als die konventionelle CTA (mit Ausnahme der Spezifität des unerfahrenen Raters, siehe Abschnitt 3.9.2). Von diesen Unterschieden waren nicht alle, aber viele statistisch signifikant. Besonders groß und signifikant war der Unterschied von früharterieller 4D-CTA und konventioneller CTA bei der Einschätzung der Übereinstimmung beider Rater (siehe Abschnitte 3.11 und 3.12) sowie bei der Sensitivität des unerfahrenen Rater 1 (siehe Abschnitt 3.9.1). Dies sind genau jene Kenngrößen, die für den unerfahrenen Rater eine Rolle spielen. Daher bietet die früharterielle 4D-CTA insbesondere für unerfahrene Ärzte und Ärztinnen sogar einen diagnostischen Mehrwert gegenüber der konventionellen CTA.

Die hier erhobenen Daten sind konsistent mit anderen Studien. Smit et al. (2015) haben hinsichtlich des Einsatzes der 4D-CTA bei der Apoplex-Diagnostik gezeigt, dass arterielle Verschlüsse ähnlich gut durch beide Verfahren detektierbar sind. Auf eine separate StandardCTA könne bei einer gleichzeitigen whole-brain-CTP-Aufnahme verzichtet werden.

Dass der Gefäßstatus in der 4D-CTA besser dargestellt werden kann, lässt sich durch die selbstkonsistente, individuelle Festlegung des optimalen Untersuchungszeitpunkt erklären. Durch die individuelle Auswertung der Kontrastmittelanreicherungskurve ist sichergestellt, dass das Gefäß ausreichend perfundiert ist. Gleichzeitig kann eine venöse Überlagerung vermieden werden und so die diagnostische Sicherheit verbessert werden. 
Eine entsprechende Optimierung des Untersuchungszeitpunktes für Standard-CTA ist theoretisch denkbar, stellt praktisch aber eine Herausforderung dar. Die Anreicherung des Kontrastmittelbolus ist schwer vorherzusagen. Eine zu starke zeitliche Abweichung nach vorne oder hinten des Untersuchungszeitpunktes vom Optimum bewirkt eine deutliche Reduktion des Kontrastes der arteriellen Darstellung. Daher raten Frölich et al. (2013) von der zeitlichen Anpassung des Standard-CTA-Protokolls ab, da dies eine 4D-CTA nicht verlässlich ersetzen kann.

Der Vorteil der Standard-CTA ist, dass sie standardmäßig verfügbar ist und die MIP automatisch und somit ohne größeren Zeitverlust angefertigt wird. Für die 4D-CTA dagegen muss individuell der Zeitpunkt der Untersuchung ausgewählt, der Datensatz in die Work Station eingeladen und die MIP angefertigt werden. Dies stellt einen Mehraufwand an Zeit und Personal dar.

Die Standard-CTA hat allerdings in ihrem Vorteil der automatischen Rekonstruktion und schnellen Verfügbarkeit auch ihren großen Nachteil: sie stellt nur eine singuläre Momentaufnahme der Kontrastmittelanreicherung dar. Das Kontrastmittel kann z. B. aber verzögert anfluten, sodass zum Untersuchungszeitpunkt der Eindruck entsteht, das Gefäß sei nicht ausreichend perfundiert. Umgekehrt kann durch einen späten Untersuchungszeitpunkt durch die venöse Überlagerung die Identifikation von Spasmen erschwert sei. Dadurch entstehen Fehlerquellen, die bei Hinzuziehen oder alleiniger Verwendung der 4D-CTA zumindest reduziert, wenn nicht sogar ausgeschaltet werden kann.

Ob eine simultane Auswertung von sowohl CTA und 4D-CTA einen diagnostischen Mehrwert bietet, bleibt zu untersuchen. Die Fragestellung, ob die Kombination beider Verfahren die diagnostische Sicherheit erhöht, war nicht im Fokus dieser Promotion.

Für den Patienten steht bezüglich der Untersuchungsroutine neben der diagnostischen Sicherheit vor Allem die Frage nach Strahlendosis und Menge des verwendeten Kontrastmittels im Vordergrund. Potentiell möglich bei Ersatz der CTA durch die 4D-CTA wäre eine Dosisreduktion der Strahlenbelastung um 6,4\% durch die Verkürzung 
der Gesamtuntersuchungszeit sowie eine Reduktion des verwendeten Kontrastmittels.

\subsection{Vergleich mit anderen Bildauswertungsprotokollen}

Aus der 4D-CTA können mit anderen Auswertungsprotokollen alternative Gefäßdarstellungen erreicht werden. In dieser Studie wurde ein individuell abgestimmter optimaler Untersuchungszeitpunkt ermittelt. An diesem Zeitpunkt wurde das dreidimensionale Bild mittels MIP entlang der Longitudinalachse auf ein zweidimensionales Bild in der Transversalebene projiziert.

\subsubsection{Einfluss des Rekonstruktionszeitpunktes}

Im Vergleich des früharteriellen und der spätarteriellen Zeitpunktes der Kontrastmittelanreicherung zur Rekonstruktion der 4D-CTA aus dem CTP-Datensatz ergibt sich ein konsistentes Bild. Ausnahmslos erreichte der früharterielle Zeitpunkt bessere Ergebnisse als der spätarterielle Zeitpunkt bei gleicher Schichtdicke. Dieser Unterschied ist signifikant für die Interrater-Reliabilitäten (Abschnitte 3.11.2 und 3.12).

\subsubsection{Einfluss der Schichtdicke}

Eine klare Präferenz zwischen den in Betracht gezogenen Schichtdicken von $5 \mathrm{~mm}$ und $10 \mathrm{~mm}$ ergibt sich aus den gefundenen Ergebnissen nicht. Je nach Kriterium sind verschiedene Schichtdicken vorteilhaft. So wurde bei einer Schichtdicke von $10 \mathrm{~mm}$ zwar eine höhere Sensitivität bei der Vasospasmusdetektion erzielt (Abschnitt 3.9.1), die Schichtdicke von $5 \mathrm{~mm}$ erlaubte aber eine höhere Spezifität (Abschnitt 3.9.2). Dieses wechselhafte Bild setzt sich durch die weiteren Ergebnisse fort. Allerdings gab es nur einen einzigen statistisch signifikanten Unterschied zwischen den Schichtdicken. Dieser ist die Pearson-Korrelation der Vasospasmusdetektion zwischen beiden Ratern für die spätarterielle 4D-CTA (Abschnitt 3.11.2). Da der früharterielle Zeitpunkt dem spätarteriellen Zeitpunkt aber ohnehin vorzuziehen ist (Abschnitt 4.3.1), fällt dieser Unterschied hier nicht ins Gewicht. 
Dass die Schichtdicke keinen systematischen Unterschied macht, ist zunächst überraschend. So würde man erwarten, dass die kleinere Schichtdicke von $5 \mathrm{~mm}$ eine bessere Ortsauflösung in axialer Richtung erlaubt, und somit eine bessere Bildbewertung ermöglicht. Eine mögliche Erklärung für das hier erzielte Resultat findet sich in der verwendeten MIP: durch Projektion in axialer Richtung werden Unterschiede in der axialen Auflösung zumindest teilweise kompensiert.

\subsubsection{Rekonstruktion mittels temporal maximum intensity projection}

Dem hier untersuchten Verfahren mit axialer MIP zu einem individuell abgestimmten Untersuchungszeitpunkt gegenüber steht eine temporal MIP (tMIP). Diese wählt keinen spezifischen Zeitpunkt aus, sondern akkumuliert Informationen über den gesamten Untersuchungszeitraum. Hierzu werden zunächst zu verschiedenen Zeitpunkten die jeweiligen dreidimensionalen Bilder mittels MIP entlang der Longitudinalachse auf zweidimensionale Bilder in der Transversalebene projiziert. Es stehen also zeitlich aufgelöste zweidimensionale Projektionen zur Verfügung. Aus diesem $(2+1)$-dimensionalen Datensatz wird mit einer weiteren MIP entlang der Zeitachse ein zweidimensionales Bild erzeugt, welches die Informationen aus allen Zeitpunkten akkumuliert.

Frölich et al. (2013) haben gezeigt, dass diese tMIP aus der 4D-CTA die Thrombuslast bei Patienten mit Verschlüssen des vorderen Kreislaufs besser aufzeigt als die Standard-CTA. Als Grund dafür nennen die Autoren die bessere Darstellung der Kollateralen.

Diese bessere Darstellung ergibt sich aus der Insensitivität auf den Untersuchungszeitpunkt, die durch die zeitliche MIP erreicht wird. Folgt man der Argumentation von Frölich et al. (2013), scheint es überraschend, dass das in dieser Promotion untersuchte Auswertungsprotokoll der Standard-CTA ähnlich überlegen ist. Es lässt sich schließen, dass die individuelle Auswahl eines optimierten Untersuchungszeitpunktes die Diagnostik genauso erleichtert wie die Akkumulation von Informationen über die Zeit. Dies kann z. B. durch venöse Überlagerung aus der spätarteriellen Phase der Bilddarstellung resultieren. Da venöse Überlagerung im Rahmen der Vasospasmusdiagnostik problematischer als für die von Frölich et al. (2013) unter- 
suchte Darstellung von Kollateralen bei Apoplex ist, könnte das in dieser Promotion untersuchte Auswertungsprotokoll sogar der tMIP überlegen sein. Endgültig kann diese Frage erst durch eine weitere Studie beantwortet werden.

\subsection{Dosisreduktion}

Die Anpassung der Untersuchungsroutine nach durch rupturierte Aneurysma verursachte SAB kann eine relevante Minderung der assoziierten Strahlendosis erreichen. Das kumulative DLP über alle Behandlungen ließe sich im Mittel um 683,1 mGy reduzieren, was einer Dosisreduktion um 6,4\% entspricht. Diese Reduktion geht nicht auf Kosten der diagnostischen Aussagekraft der Untersuchungen, sondern geht sogar mit einer verbesserten Aussagekraft einher (siehe Abschnitt 4.2.1).

Diese Dosisreduktion ist umso relevanter, da besonders jüngere Patienten betroffen sind. Auf den ersten Blick mag eine Reduktion um $6,4 \%$ wenig beeindruckend erscheinen. Jedoch sollte hier in Betracht gezogen werden, dass nach Brenner und Hall (2009) bis zu 2\% zukünftiger Tumore auf CT-assoziierte stochastische Strahlenschäden zurückzuführen sind. Diese Strahlenschäden entstehen zum einen durch direkte Schädigung der Desoxyribonukleinsäuren (DNA) durch ionisierende Röntgenstrahlung. Zum anderen interagiert die Röntgenstrahlung mit Wassermolekülen im Körper und führt so zur Entstehung von Radikalen, die z. B. für DNA-Strangbrüche verantwortlich sind. In der Regel können solche Defekte automatisch repariert werden. Potentielle Fehlreparaturen können aber Punktmutationen, chromosomale Translokationen oder Fusionen bedingen. Diese wiederum sind z.T. assoziiert mit der Entstehung maligner Tumore. Für stochastische Zellschäden gibt es keinen Dosisschwellenwert, sondern es gilt: mit zunehmender Höhe der Strahlendosis steigt die Wahrscheinlichkeit des Eintretens eines Strahlenschadens (Bundesamt für Strahlenschutz). Da die Hälfte der in dieser Promotion untersuchten Patienten unter 54 Jahre alt ist (siehe Abschnitt 3.1), ist eine hohe im weiteren Leben akkumulierte Strahlenbelastung zu erwarten. Dies macht die Verminderung der Dosis umso wichtiger, sodass die diagnostisch vertretbaren 6,4\% Dosisreduktion relevant sind. 
Darüber hinaus ist durch technischen Fortschritt eine weitere Dosisreduktion zu erwarten. Die Gesamtstrahlendosis der 4D-CTA errechnet sich als Summe der individual acquisitions. Auch wenn momentan für die einzelnen Akquisitionen weniger Strahlendosis benötigt wird als für die Standard-CTA ist die Gesamtstrahlendosis der 4DCTA in der Regel in der Summe höher als für die Standard-CTA (Kortman et al. 2015). Eine weitere Verminderung der Einzeldosen der individual acquisitions geht zulasten der Signal-to-noise-ratio, d. h. auf Kosten höheren Bildrauschens. Eine Rauschfilterung durch iterative Bildrekonstruktion kann das Bildrauschen reduzieren, sodass bei Verminderung der Dosis trotzdem die gleiche Bildqualität erzielt werden kann (Kortman et al. 2015). Aus dieser Verminderung der Einzeldosen wird also auch eine Verminderung der Gesamtdosis der 4D-CTA erzielt werden können.

\subsection{Datenlage für die hintere Zirkulation}

Sämtliche Fragestellungen dieser Promotion waren für die Arterien der hinteren Zirkulation, also für BA und PA, nicht suffizient zu beantworten. Dies kann dadurch erklärt werden, dass die vom CTScanner erfasste sogenannte whole-brain-Perfusion lediglich einen Scanbereich von $10 \mathrm{~cm}$ um den Circulus Wilisii darstellen kann. Im Rahmen dieser Promotion wurde das Gebiet so ausgewählt, dass insbesondere die Arterien des vorderen Kreislaufs, die ACA, MCA, ACOM, ICA gut darstellbar waren. Dies erscheint angesichts der Lokalisation der überwiegenden Mehrzahl der Aneurysmen (und damit auch der erwarteten Komplikationen) vertretbar. Nichtsdestotrotz sind die Daten zur PA und BA schlecht auswertbar. Diese Arterien sind häufig so schlecht in den Rekonstruktionen abgebildet, dass nicht ausreichend verwertbare Ratings vorliegen, um eine aussagekräftige Stellungnahme zur Detektion der Vasospasmen in diesen Arterien treffen zu können. Im Rahmen einer weiteren Studie mit Fokus auf der hinteren Zirkulation würde sich eine dahingehende Untersuchung empfehlen. Unter den gegeben technischen Voraussetzungen kommt auch die Studie von Smit et al. (2015) zu diesem Schluss. 


\subsection{Limitationen der Studie}

Die im Rahmen dieser Promotion durchgeführte Studie hat multiple Limitationen. Zum einen ist das Patientenkollektiv für statistische Auswertungen relativ klein. So ist die Stichprobengröße von knapp 40 Patienten an der unteren Grenze für die Anwendbarkeit von statistischen Aussagen zur Signifikanz. Zum einen wurde dieser Einschränkung beigekommen, indem Konfidenzintervalle und darauf basierend Signifikanz sehr konservativ und mit Korrekturen für kleine Stichprobengrößen geschätzt wurden. Zum anderen macht die gute demographische Übereinstimmung des Patientenkollektivs der SAB mit anderen Studienergebnissen in der Literatur Hoffnung, dass das Kollektiv trotz seiner Größe repräsentativ ist. Da sich dieses Patientenkollektiv aus allen Patienten, die seit 2013 an der UMG die Kriterien erfüllt haben, zusammensetzt, blieben nur zwei Möglichkeiten, das Kollektiv zu erweitern: (1) der Studienzeitraum könnte verlängert werden. Aufgrund sich entwickelnder Technik und angepasster Behandlungsprotokolle ist die Homogenität über einen noch längeren Zeitraum allerdings schwer gegeben. (2) Die Studie könnte Fälle von mehreren Häusern mit einbeziehen. Hier müssten allerdings vergleichbare technische Voraussetzungen und Behandlungsprotokolle im Vorfeld koordiniert werden.

Zum anderen handelt es sich von der Anlage der Studie um einen retrospektiven Ansatz. Durch die Auswahl der Patienten besteht ein Bias insofern, als dass das Patientenkollektiv die tatsächliche Inzidenz von Vasospasmen nach SAB nicht exakt wiedergibt. Dadurch könnte der positiv prädiktive Wert etwas höher als in der Realität sein. Die Studie versuchte, diesem Bias zu begegnen, indem Patienten ohne bekannten interventionell behandlungsbedürftigen Vasospasmus mit in das Kollektiv aufgenommen wurden. Da für diese Patienten jedoch keine DSA vorliegt, kann das Fehlen eines interventionell behandlungsbedürftigen Vasospasmus nicht durch den Goldstandard validiert werden, sondern muss als so gegeben angenommen werden. Dieser Limitierung könnte man nur durch eine prospektive Studie aufheben, die eine DSA ins Standardprotokoll nach SAB aufnehmen würde. Eine DSA ohne medizinische Indikation wäre ethisch allerdings kaum vertretbar. 
Aufgrund der schweren Auswertbarkeit der BA und PA (vgl. Abschnitt 3.3) sind die Ergebnisse für diese Arterien mit großer Unsicherheit versehen. Somit beschränken sich die belastbaren Ergebnisse dieser Studie im Prinzip auf ACOM, ACA, ICA und MCA. Da diese Limitierung eine direkte Folge der Bildgebungsqualität ist, könnte in Zukunft die Verwendung von verschiedenen Fokusbereichen dieses Defizit ausgleichen. 


\section{Zusammenfassung}

Ziel dieser Arbeit war die Beantwortung der Frage, ob in der SOP zur Diagnose von Vasospasmen nach durch Aneurysma verursachte SAB auf die CTA zugunsten einer alleinigen CTP verzichtet werden kann. Hierzu wurde eine retrospektive Studie aller seit 2013 an der UMG infrage kommenden Patienten durchgeführt. Die daraus resultierende Stichprobe ist mit 31 Patienten mit entsprechendem Vasospasmus und 5 Kontrollpatienten ohne interventionell behandlungsbedürftigen Vasospasmus verhältnismäßig klein. Zum einen wurde dies bei der statistischen Auswertung berücksichtigt, zum anderen stimmt die Stichprobe trotz ihrer limitierten Größe hinsichtlich epidemologischer und demographischer Kenngrößen mit in der Litaratur referenzierten Stichproben sehr gut überein. Daher ist davon auszugehen, dass die Ergebnisse dieser Studie dennoch repräsentativ sind.

Die eigentliche Fragestellung dieser Arbeit konnte eindeutig beantwortet werden: die CTA kann ohne Einschränkungen des diagnostischen Wertes für Gefäße der vorderen Zirkulation durch die alleinige CTP bzw. 4D-CTA aus CTP ersetzt werden. Keine der untersuchten Charakterisierungen der Testqualität stellte sich für die 4D-CTA schlechter dar als für die CTA. Für den Zeitpunkt der Rekonstruktion war die früharterielle Darstellung der spätarteriellen fast ausnahmslos überlegen, sodass diese zu empfehlen ist. Bei Verwendung der früharteriellen Darstellung ist die 4D-CTA der konventionellen CTA sogar überlegen, insbesondere für unerfahrene Ärzte: hier ergab sich ein signifikanter Anstieg der Sensitivität und der Übereinstimmung mit einem erfahrenen Arzt. Die Spezifität der 4D-CTA war gleichzeitig nicht signifikant schlechter als für die konventionelle CTA.

Bei Verzicht auf die konventionelle CTA im SOP lässt sich zudem eine mittlere Dosisreduktion von ca. $6 \%$ erzielen. 


\section{Referenzen}

Agid R, Lee SK, Willinsky RA, Farb RI, terBrugge KG (2006): Acute subarachnoid hemorrhage: Using 64-slice multidetector CT angiography to "triage“ patients' treatment. Neuroradiology $\underline{48}$, 787-794

Anxionnat R, Bracard S, Macho J, da Costa E, Vaillant R, Launay L, Trousset Y, Romeas R, Picard L (1998): 3D angiography. Clinical interest. First applications in interventional neuroradiology. J Neuroradiol 25, 251-62

Baumgartner RW, Arnold M, Gönner F, Staikow I, Herrmann C, Rivoir A, Müri RM (1997a): Contrast-enhanced transcranial color-coded duplex sonography in ischemic cerebrovascular disease. Stroke 28, 2473-2478

Baumgartner RW, Baumgartner I, Mattle HP, Schroth G (1997b): Transcranial color-coded duplex sonography in the evaluation of collateral flow through the circle of Willis. Am J Neuroradiol 18, 127-133

Boulouis G, Labeyrie MA, Raymond J, Rodriguez-Régent C, Lukaszewicz AC, Bresson D, Ben Hassen W, Trystram D, Meder JF, Oppenheim C, Naggara O (2017): Treatment of cerebral vasospasm following aneurysmal subarachnoid haemorrhage: a systematic review and meta-analysis. Eur Radiol 27, 3333-3342

Brenner DJ, Hall EJ (2009): Computed tomography - an increasing source of radiation exposure. Yearb Pulm Dis 2009, 154-155

Brouwer PA, Bosman T, Van Walderveen MAA, Krings T, Leroux AA, Willems PWA (2010): Dynamic 320-section CT angiography in cranial arteriovenous shunting lesions. Am J Neuroradiol 31, 767-770

Bundesamt für Strahlenschutz: Ionisierende Strahlung. https//www.bfs.de/DE/themen/ion/wirkung/einfuehrung/einfuehrung.html; abgerufen am 11.09.2019

Charpentier C, Audibert G, Guillemin F, Civit T, Ducrocq X, Bracard S, Hepner H, Picard L, Laxenaire MC (1999): Multivariate analy- 
sis of predictors of cerebral vasospasm occurrence after aneurysmal subarachnoid hemorrhage. Stroke 30, 1402-1408

da Costa LB, Gunnarsson T, Wallace MC (2004): Unruptured intracranial aneurysms: natural history and management decisions. Neurosurg Focus 17, 1-6

da Costa LB, de Morais JV, de Andrade A, Vilela MD, Pontes RPC, Braga BP (2005): Surgical treatment of intracranial aneurysms: six-year experience in Belo Horizonte, MG, Brazil. Arq Neuropsiquiatr $\underline{62}, 245-249$

de Rooij NK, Linn FHH, van der Plas SAP, Algra A, Rinkel GJE (2007): Incidence of subarachnoid haemorrhage: a systematic review with emphasis on region, age, gender and time trends. J Neurol Neurosurg Psychiatry $\underline{78}, 1365-1372$

Dorsch NWC, King MT (1994): A review of cerebral vasospasm in aneurysmal subarachnoid haemorrhage Part I: Incidence and effects. J Clin Neurosci 1, 19-26

Elsayed AA, Moran CJ, Cross DT, Derdeyn CP, Pilgram TK, Milburn JM, Dacey RG, Diringer MN (2006): Effect of intraarterial papaverine and/or angioplasty on the cerebral veins in patients with vasospasm after subarachnoid hemorrhage due to ruptured intracranial aneurysms. Neurosurg Focus 21, E16

Feigin VL, Lawes CMM, Bennett DA, Barker-Collo SL, Parag V (2015): Worldwide stroke incidence and early case fatality reported in 56 population-based studies: a systematic review. Lancet Neurol $\underline{8}$, 355-369

Ferguson S, Macdonald RL (2007): Predictors of cerebral infarction in patients with aneurysmal subarachnoid hemorrhage. Neurosurgery $\underline{60}, 658-667$

Fieller AEC, Hartley HO, Pearson ES (1957): Tests for rank correlation coefficients. Biometrika $\underline{44}, 470-481$

Findlay JM, Nisar J, Darsaut T (2016): Cerebral vasospasm: A review. Can J Neurol Sci $\underline{43}, 15-32$

Fisher CM, Roberson GH, Ojemann RG (1977): Cerebral vasospasm with ruptured saccular aneurysm-the clinical manifestations. Neurosurgery 1 , 245-248 
Fisher CM, Kistler JP, Davis JM (1980): Relation of cerebral vasospasm to subarachnoid hemorrhage visualized by computerized tomographic scanning. Neurosurgery $\underline{6}$, 1-9

Frölich AMJ, Schrader D, Klotz E, Schramm R, Wasser K, Knauth M, Schramm P (2013): 4D CT angiography more closely defines intracranial thrombus burden than single-phase CT angiography. Am J Neuroradiol 34, 1908-1913

Graf CJ (1971): Prognosis for patients with nonsurgically-treated aneurysms: analysis of the Cooperative Study of Intracranial Aneurysms and Subarachnoid Hemorrhage. J Neurosurg 35, 438-443

Gray H: Anatomy of the human body. 20. Auflage; Lea \& Febiger, Philadelphia 1918

Haley Jr EC, Kassell NF, Torner JC (1993): A randomized trial of nicardipine in subarachnoid hemorrhage: angiographic and transcranial Doppler ultrasound results. A report of the Cooperative Aneurysm Study. J Neurosurg $\underline{78}$, 548-553

Hennerici M (2015): Diagnostik akuter zerebrovaskulärer Erkrankungen - Leitlinien für Diagnostik und Therapie in der Neurologie. Dtsch Gesellschaft für Neurol 1-14

Heros RC, Zervas NT, Varsos V (1983): Cerebral vasospasm after subarachnoid hemorrhage: an update. Ann Neurol 14, 599-608

Hijdra A, Van Gijn J, Stefanko S, Van Dongen KJ, Vermeulen M, Van Crevel H (1986): Delayed cerebral ischemia after aneurysmal subarachnoid hemorrhage Clinicoanatomic correlations. Neurology $\underline{36}, 329$

Hoh BL, Topcuoglu MA, Singhal AB, Pryor JC, Rabinov JD, Rordorf GA, Carter BS, Ogilvy CS (2004): Effect of clipping, craniotomy, or intravascular coiling on cerebral vasospasm and patient outcome after aneurysmal subarachnoid hemorrhage. Neurosurgery 55, 779-786

Hohlrieder M, Spiegel M, Hinterhoelzl J, Engelhardt K, Pfausler B, Kampfl A, Ulmer H, Waldenberger P, Mohsenipour I, Schmutzhard E (2002): Cerebral vasospasm and ischaemic infarction in clipped and coiled intracranial aneurysm patients. Eur J Neurol $\underline{9}, 389-399$ 
Hop JW, Rinkel GJE, Algra A, van Gijn J (1997): Case-fatality rates and functional outcome after subarachnoid hemorrhage. Stroke $\underline{28}, 660-664$

Kahl-Scholz M, Vockelmann C (Hrsg.): Basiswissen Radiologie (Springer-Lehrbuch). Springer, Berlin 2017

Knauth M, Von Kummer R, Jansen O, Hähnel S, Dörfler A, Sartor K (1997): Potential of CT angiography in acute ischemic stroke. Am J Neuroradiol 18, 1001-1010

Kortman HGJ, Smit EJ, Oei MTH, Manniesing R, Prokop M, Meijer FJA (2015): 4D-CTA in neurovascular disease: A review. Am J Neuroradiol 36, 1026-1033

Landis JR, Koch GG (1977): The measurement of observer agreement for categorical data. Biometrics $\underline{33}, 159-174$

Lanzino G, Kassell NF, Participants (1999): Double-blind, randomized, vehicle-controlled study of high-dose tirilazad mesylate in women with aneurysmal subarachnoid hemorrhage. Part II. A cooperative study in North America. J Neurosurg 90, 1018-1024

Leitlinie Subarachnoidalblutung. Deutsche Gesellschaft für Neurologie 2012. https://www.awmf.org/uploads/tx_szleitlinien/030073l_S1_Subarachnoidalblutung_2012_abgelaufen.pdf; abgerufen am 11.09.2019

Li H, Pan R, Wang H, Rong X, Yin Z, Milgrom DP, Shi X, Tang Y, Peng $Y$ (2013): Clipping versus coiling for ruptured intracranial aneurysms: A systematic review and meta-analysis. Stroke 44, 29-37

Linn FHH, Rinkel GJE, Algra A, van Gijn J (1996): Incidence of subarachnoid hemorrhage. Stroke 27, 625-629

Longstreth WT (1994): Subarachnoid hemorrhage and hormonal factors in women: A population-based case-control study. Ann Intern Med 121, 168

Lysakowski C, Walder B, Costanza MC, Tramèr MR (2001): Transcranial Doppler versus angiography in patients with vasospasm due to a ruptured cerebral aneurysm: a systematic review. Stroke $\underline{32}$, $2292-2298$

Macdonald RL, Higashida RT, Keller E, Mayer SA, Molyneux A, Raabe A, Vajkoczy P, Wanke I, Bach D, Frey A (2011): Clazosentan, an endothelin receptor antagonist, in patients with aneurysmal 
subarachnoid haemorrhage undergoing surgical clipping: a randomised, double-blind, placebo-controlled phase 3 trial (CONSCIOUS-2). Lancet Neurol 10, 618-625

Malinova V, Tsogkas I, Behme D, Rohde V, Psychogios MN, Mielke D (2020): Defining cutoff values for early prediction of delayed cerebral ischemia after subarachnoid hemorrhage by CT perfusion. Neurosurg Rev 43, 581-587

Mees SD, Rinkel GJ, Feigin VL, Algra A, van den Bergh WM, Vermeulen M, van Gijn J (2007): Calcium antagonists for aneurysmal subarachnoid haemorrhage. Cochrane Database Syst Rev $\underline{3}$, 1465-1858

Mhurchu CN, Anderson C, Jamrozik K, Hankey G, Dunbabin D (2011): Hormonal factors and risk of aneurysmal subarachnoid hemorrhage. Stroke $\underline{32}$, 606-612

Mijiti M, Mijiti P, Axier A, Amuti M, Guohua Z, Xiaojiang C, Kadeer K, Xixian W, Geng D, Maimaitili A (2016): Incidence and predictors of angiographic vasospasm, symptomatic vasospasm and cerebral infarction in chinese patients with aneurysmal subarachnoid hemorrhage. PLoS One 11, e0168657

Molyneux AJ, Kerr RS, Yu L, Clarke M, Sneade M, Yarnold JA, Sandercock P (2005): International subarachnoid aneurysm trial (ISAT) of neurosurgical clipping versus endovascular coiling in 2143 patients with ruptured intracranial aneurysms: a randomised comparison of effects on survival, dependency, seizures, rebleeding, subgroups, and . Lancet 366, 809-817

Molyneux AJ, Kerr RS, Birks J, Ramzi N, Yarnold J, Sneade M, Rischmiller J (2009): Risk of recurrent subarachnoid haemorrhage, death, or dependence and standardised mortality ratios after clipping or coiling of an intracranial aneurysm in the International Subarachnoid Aneurysm Trial (ISAT): long-term follow-up. Lancet Neurol $\underline{8}, 427-433$

Molyneux AJ, Birks J, Clarke A, Sneade M, Kerr RSC (2015): The durability of endovascular coiling versus neurosurgical clipping of ruptured cerebral aneurysms: 18 year follow-up of the UK cohort of the International Subarachnoid Aneurysm Trial (ISAT). Lancet $\underline{385}, 691-697$ 
Pedersen HK, Bakke SJ, Hald JK, Skalpe IO, Anke IM, Sagsveen R, Langmoen IA, Lindegaard KF, Nakstad PH (2001): CTA in patients with acute subarachnoid haemorrhage: A comparative study with selective, digital angiography and blinded, independent review. Acta radiol $\underline{42}, 43-49$

Petridis AK, Kamp MA, Cornelius JF, Beez T, Beseoglu K, Turowski B, Steiger HJ (2017): Aneurysmal subarachnoid hemorrhage-diagnosis and treatment. Dtsch Arztebl Int 114, 226-235

Purkayastha S, Farzaneh F (2014): Transcranial Doppler ultrasound: Technique and application. Semin Neurol 32, 411-420

Rabinstein AA, Friedman JA, Weigand SD, McClelland RL, Fulgham JR, Manno EM, Atkinson JLD, Wijdicks EFM (2004): Predictors of cerebral infarction in aneurysmal subarachnoid hemorrhage. Stroke $\underline{35}, 1862-1866$

Raper DMS, Allan R (2010): International Subarachnoid Trial in the long run. Neurosurgery $\underline{66}, 1166-1169$

Reichenbach JR, Röther J, Jonetz-Mentzel L, Herzau M, Fiala A, Weiller C, Kaiser WA (1999): Acute stroke evaluated by time-to-peak mapping during initial and early follow-up perfusion CT studies. AJNR Am J Neuroradiol 20, 1842-1850

Reiser M, Kuhn F-P, Debus J (Hrsg.): Duale Reihe Radiologie. Vierte Auflage; Georg Thieme Verlag, Stuttgart 2017

Rinkel GJE, Djibuti M, Algra A, Van Gijn J (1998): Prevalence and risk of rupture of intracranial aneurysms: a systematic review. Stroke 29, 251-256

Rinne H: Taschenbuch der Statistik. Harri Deutsch Verlag, Frankfurt am Main 2008

Rowland MJ, Hadjipavlou G, Kelly M, Westbrook J, Pattinson KTS (2012): Delayed cerebral ischaemia after subarachnoid haemorrhage: Looking beyond vasospasm. Br J Anaesth 109, 315-329

Salomon EJ, Barfett J, Willems PWA, Geibprasert S, Bacigaluppi S, Krings T (2009): Dynamic CT angiography and CT perfusion employing a 320-detector row CT. Clin Neuroradiol 19, 187-196

Schregel K, Tsogkas I, Peter C, Zapf A, Behme D, Schnieder M, Maier IL, Liman J, Knauth M, Psychogios MN (2018): Outcome prediction using perfusion parameters and collateral scores of multi-pha- 
se and single-phase CT angiography in acute stroke: need for one, two, three, or thirty Scans? J Stroke 20, 362-372

Seabold S, Perktold J: statsmodels: Econometric and statistical modeling with python. In: van der Walt S, Millmann J (Hrsg.): Proceedings of the 9th Python in Science Conference. 2010, 92-96

Sedat J, Dib M, Lonjon M, Litrico S, Von Langsdorf D, Fontaine D, Paquis $P$ (2002): Endovascular treatment of ruptured intracranial aneurysms in patients aged 65 years and older: Follow-up of 52 patients after 1 year. Stroke 33, 2620-2625

Seidel G, Kaps M, Gerriets T (1995): Potential and limitations of transcranial color-coded sonography in stroke patients. Stroke $\underline{26}$, 2061-2066

Smit EJ, Vonken E-J, van der Schaaf IC, Mendrik a. M, Dankbaar JW, Horsch a. D, van Seeters T, van Ginneken B, Prokop M (2012): Timing-invariant reconstruction for deriving high-quality CT angiographic data from cerebral CT perfusion data. Radiology 263, 216-225

Smit EJ, Vonken E-J, Meijer F, Dankbaar J, Horsch A, van Ginneken B, Velthuis B, van der Schaaf I, Prokop M (2015): Timing-invariant CT angiography derived from CT Perfusion imaging in acute stroke: A diagnostic performance study. Am J Neuroradiol $\underline{36}$, 18341838

Spendel MC (2008): Die aneurysmatische Subarachoidalblutung: Epidemiologie, Ätiologie, Klinik und Komplikationen. Neurol Neurochir und Psychiatr $\underline{9}$, 20-30

van Gijn J, Rinkel GJ (2001): Subarachnoid haemorrhage: diagnosis, causes and management. Brain 124, 249-278

van Gijn J, Kerr RS, Rinkel GJE (2007): Subarachnoid haemorrhage. Lancet $\underline{369}, 306-318$

Velat GJ, Kimball MM, Mocco JD, Hoh BL (2011): Vasospasm after aneurysmal subarachnoid hemorrhage: Review of randomized controlled trials and meta-analyses in the literature. World Neuros$\operatorname{urg} \underline{76}, 446-454$

Vergouwen MDI, Vermeulen M, van Gijn J, Rinkel GJE, Wijdicks EF, Muizelaar JP, Mendelow AD, Juvela S, Yonas H, Terbrugge KG, et al. (2010): Definition of delayed cerebral ischemia after aneurysmal subarachnoid hemorrhage as an outcome event in clinical 
trials and observational studies: proposal of a multidisciplinary research group. Stroke $\underline{41}$, 2391-2395

Widder B, Hamann GF (Hrsg.): Duplexsonographie der hirnversorgenden Arterien. Siebte Auflage; Springer, Berlin 2018

Yang C-Y, Chen Y-F, Lee C-W, Huang A, Shen Y, Wei C, Liu H-M (2008): Multiphase CT angiography versus single-phase CT angiography: comparison of image quality and radiation dose. Am J Neuroradiol 29, 1288-1295

Yoshimoto Y, Wakai S, Satoh A, Tejima T, Hamano M (1999): A prospective study on the effects of early surgery on vasospasm after subarachnoid hemorrhage. Surg Neurol 1ㅗ, 392-398

Zhao B, Xiong Y, Yin R, Zheng K, Li Z, Tan X, Yang H, Zhong M, Group AS (2016): Aneurysm rebleeding after poor-grade aneurysmal subarachnoid hemorrhage: predictors and impact on clinical outcomes. J Neurol Sci 371, 62-66

Zhao J, Lin H, Summers R, Yang M, Cousins BG, Tsui J (2017): Current treatment strategies for intracranial aneurysms: An overview. Angiology $\underline{69}, 17-30$

Zimmermann SF: Doppler- (TCD) und Duplexsonographie (TCCD) in der Diagnostik und im Therapieverlauf von Vasospasmen nach aneurysmatischer Subarachnoidalblutung. Med. Diss. Hamburg 2010 